\title{
Die known-item search in Bibliothekskatalogen - Nutzerverhalten, Probleme, Lösungsmöglichkeiten
}

\author{
The known-item search in library catalogues - \\ user behaviour, problems, solution possibilities
}

\author{
Bachelorarbeit im Studiengang \\ Bibliotheks- und Informationsmanagement
}

an der Hochschule der Medien Stuttgart

vorgelegt von

Matthias Krebs

am

14.02.2012

Erstprüferin: Prof. Heidrun Wiesenmüller

Zweitprüfer: Prof. Markus Hennies 


\section{Kurzfassung}

Diese Bachelorarbeit untersucht das Nutzerverhalten bei einer known-item search, der Suche nach einem bekannten Objekt, im Bibliothekskatalog.

Die vorliegenden Logfiles stammen aus dem Landesbibliothekszentrum RheinlandPfalz. Für einen Vergleich wurden zudem Logfiles aus der UB Heidelberg herangezogen.

Untersucht werden Eingaben, Durchführung und Ergebnisse bei Recherchen mittels Titel, Verfasser, ISBN, Signatur und Kombinationen von Suchfeldern in der erweiterten Suche. Zudem wird der Einsatz von Booleschen Operatoren und Trunkierungen näher beleuchtet.

Da dieses Themengebiet noch nie in diesem Umfang abgedeckt wurde, ist ein Vergleich mit der gängigen Literatur schwer. Der Großteil an Literatur untersucht die thematische Suche, die Verteilung der Anfragen auf Suchfelder oder den Aufbau der Onlinehilfe.

Eine anschließende Analyse und Interpretation möglicher Gründe für Recherchevorgänge führt zu Verbesserungsvorschlägen.

Eine parallel durchgeführte Umfrage mittels Fragebogen stellt den Praxisbezug her; gleichzeitig lassen sich die Ergebnisse der Befragung mit den Logfiles vergleichen.

Schlagwörter:

Logfiles; known-item search; Analyse; Vergleich; Landesbibliothekszentrum Rheinland-Pfalz; Universitätsbibliothek Heidelberg; Bibliothekskatalog 


\section{Abstract}

This Bachelor Thesis examines the progress of users undertaking a known-item search, the search for a known object in a library catalogue.

The Logfiles used in this thesis are from the regional library center in RhinelandPalatinate "Landesbibliothekszentrum Rheinland-Pfalz". For a comparison, Logfiles from the Heidelberg University Library were consulted. Before they were examined, the received Logfiles had to be converted into a readable format.

The entry, execution and results using the title, author, ISBN, signature and combination of look-up fields of the expanded search have been examined. In addition the application of Boolean operators and truncations were highlighted.

A comparison with common literature is difficult, because this subject was not covered previously using this approach. A good portion of the literature examines the thematic search, the apportionment of queries in look-up fields or the assembly of online help.

The analysis of these factors related to searches has identified a number of suggestions for improvements.

The relationship to the code of practice is presented from surveys obtained via questionnaires. After the evaluation the results are compared to the Logfiles.

Keywords:

Logfiles; known-item search; analysis; comparison; Landesbibliothekszentrum Rheinland-Pfalz; Heidelberg University Library; library catalogue 


\section{Inhaltsverzeichnis}

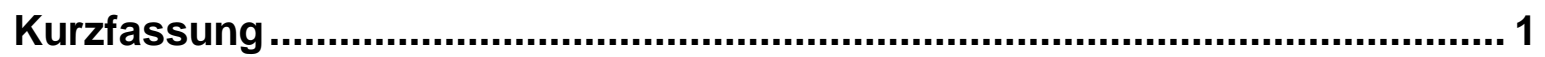

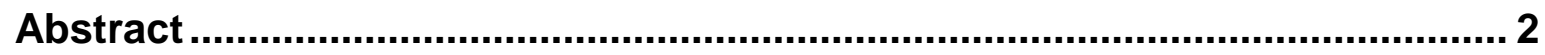

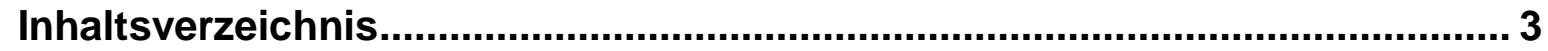

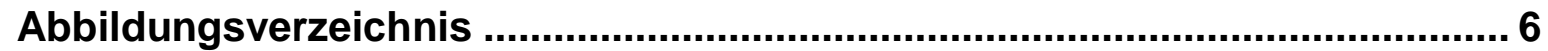

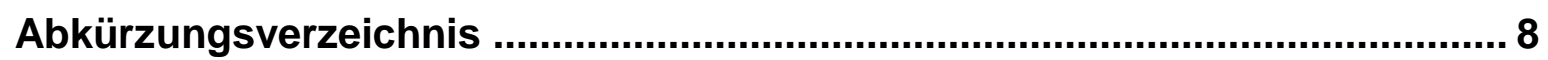

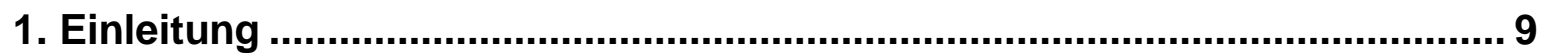

2. Aktueller Forschungsstand ....................................................................... 12

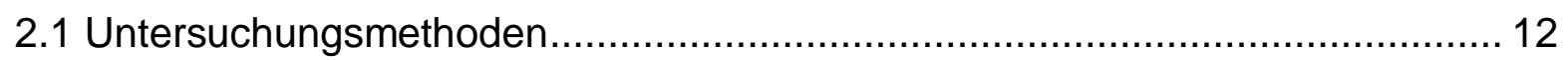

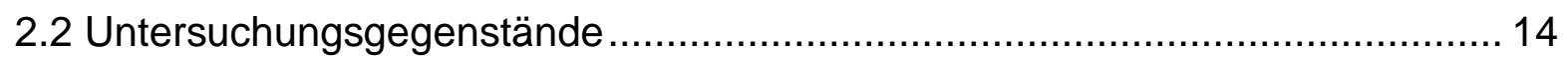

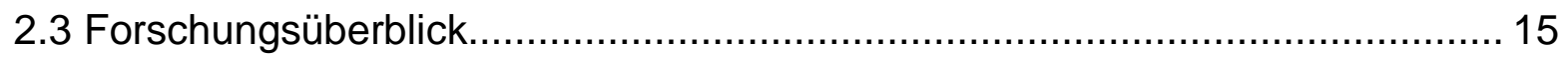

3. Methodische Vorgehensweise ............................................................... 18

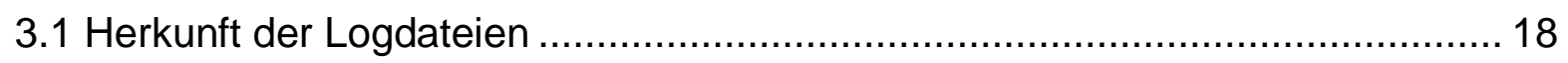

3.2 Inhalte der Logfiles ................................................................... 23

3.3 Umwandlung der Logfiles in Klarschrift................................................. 25

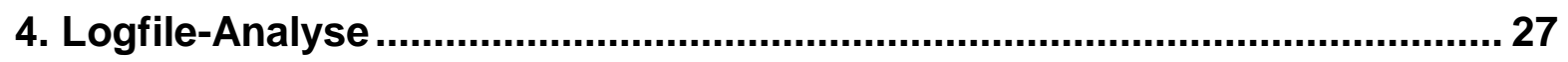

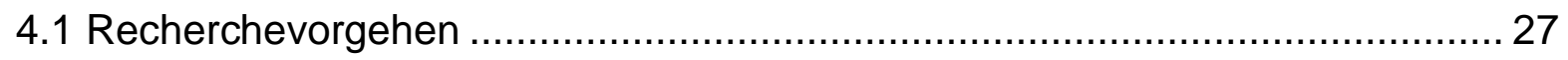

4.2 Auffällige Verhaltensmuster bei der Recherche........................................ 32

4.3 Rechercheverhalten im Katalog der UB Heidelberg ................................... 51

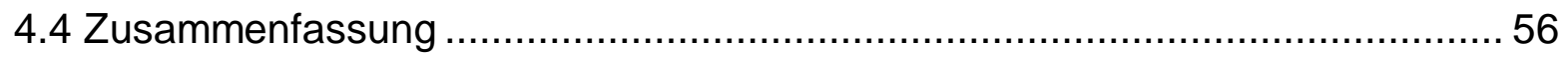


5.1 Recherchevorgänge und ihre Intentionen ............................................. 58

5.2 Verbesserungsmöglichkeiten der Recherche ....................................... 62

5.3 Positive und negative Rechercheaspekte des LBZ Rheinland-Pfalz OPACs ... 67

5.4 Spezifika des Heidelberger OPACs ..................................................... 69

5.5 Untersuchungsergebnisse im Kontext der Literatur ............................... 70

6. Erfahrungen aus der Praxis ................................................................... 71

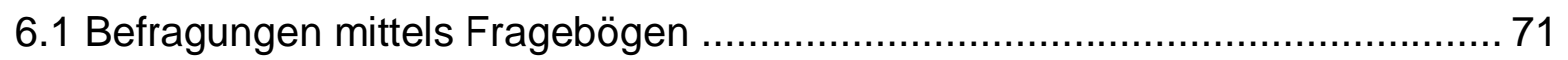

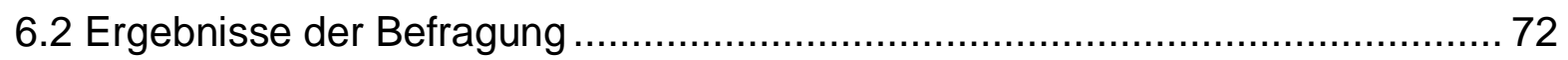

6.3 Rückschlüsse und Vergleich mit den Logfiles......................................... 74

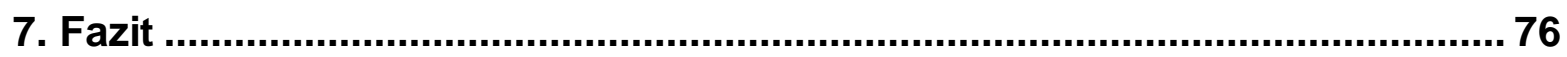

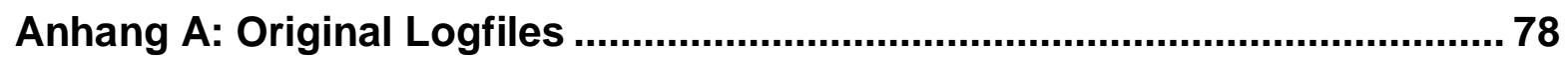

A.1a: Logfiles des LBZ Rheinland-Pfalz vom 18.10.2011 ............................. 78

A.1b: Logfiles des LBZ Rheinland-Pfalz vom 24.10.2011 .............................. 80

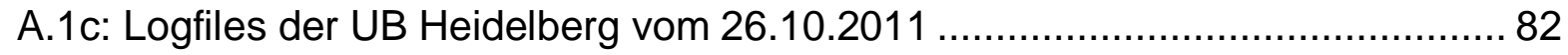

A.1d: Logfiles der UB Heidelberg vom 05.11.2011 ......................................... 84 
B.1a: Umgewandelte Logfiles des LBZ Rheinland-Pfalz vom 18.10.2011 _........... 86

B.1b: Umgewandelte Logfiles des LBZ Rheinland-Pfalz vom 24.10.2011 ........... 88

B.1c: Umgewandelte Logfiles der UB Heidelberg vom 26.10.2011 ................... 90

B.1d: Umgewandelte Logfiles der UB Heidelberg vom 05.11.2011 ....................92

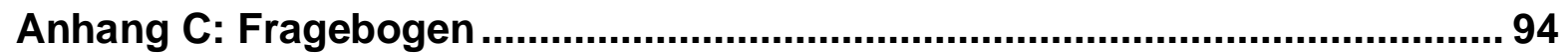

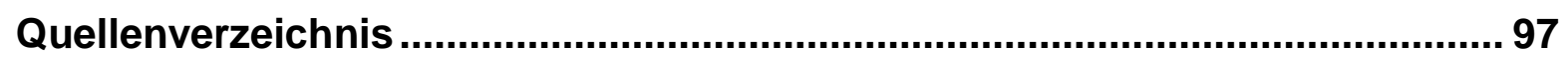

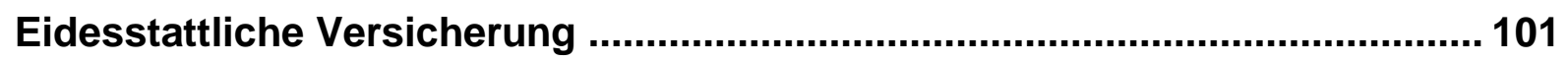




\section{Abbildungsverzeichnis}

Abbildung 1: Suchmaske des LBZ-Katalogs.

(Quelle: http://kat.lbz-

rlp.de/webOPACClient/start.do?Login=plb\&amp;BaseURL=http://www.l bz-rlp.de/cms/literatursuche/lbz-katalog/index.html / Zugriff:

01.02.2012)

Abbildung 2: LBZ-Katalog: Eingrenzung der Suche 21

(Quelle: http://kat.lbz-

rlp.de/webOPACClient/start.do?Login=plb\&amp;BaseURL=http://www.l bz-rlp.de/cms/literatursuche/lbz-katalog/index.html / Zugriff:

01.02.2012)

Abbildung 3: LBZ-Katalog: Sucheinstellungen 21

(Quelle: http://kat.llbz-

rlp.de/webOPACClient/search.do?methodToCall=submit\&CSId=3845N 28S8ba7b3ffd96de7fe8cfe3341da4c2a8bc706cf9e\&methodToCallPara meter $=$ searchPreferences \&searchCategories $[0]=-$

1\&searchString[0]=\&combinationOperator[1]=AND\&searchCategories[1 l=331\&searchString[1]=\&combinationOperator[2]=AND\&searchCategor ies[2]=100\&searchString[2]=\&combinationOperator[3]=AND\&searchCat egories[3]=902\&searchString[3]=\&callingPage=searchParameters\&sel ectedViewBranchlib=1\&searchRestrictionID[0] $=5 \&$ searchRestrictionVal ue1[0]=\&searchRestriction ID[1]=4\&searchRestrictionValue1[1]=\&searc hRestrictionID[2]=2\&searchRestrictionValue1[2]=\&searchRestrictionVal ue2[2]=\&searchRestrictionID[3]=1\&searchRestrictionValue1[3]= / Zugriff: 01.02.2012)

Abbildung 4: Die erweiterte Suche in HEIDI 23

(Quelle: http://katalog.ub.uni-heidelberg.de/cgibin/search.cgi?art=f\&sess=596e5d543ad25fca13437fd976858bb1 / Zugriff: 01.02.2012)

Abbildung 5: LBZ-Katalog: Einfache Suche auf der Seite des Landesbibliothekszentrums / Pfälzische Landesbibliothek 28 (Quelle: http://www.lbz-rlp.de/cms/plb/index.html?no cache=1 / Zugriff: $01.02 .2012)$ 
Abbildung 6: LBZ-Katalog: Direkter Weg zum OPAC

(Quelle: http://www.lbz-

rlp.de/cms/literatursuche/katalog/index.html?no cache=1/ Zugriff:

01.02.2012)

Abbildung 7: LBZ-Katalog: Suchfenster mit freier Suche 29

(Quelle: http://kat.lbz-

rlp.de/webOPACClient/start.do?Login=plb\&amp;BaseURL=http://www.l bz-rlp.de/cms/literatursuche/lbz-katalog/index.html / Zugriff:

01.02.2012)

Abbildung 8: Prozentuale Verteilung der Suchoptionen in der erweiterten Suche 30

Abbildung 9: Prozentuale Darstellung der Booleschen Operatoren

bei der erweiterten Suche. 31

Abbildung 10: Prozentuale Darstellung der Treffermenge 32

Abbildung 11: HEIDI. Rechts unten die Möglichkeit 'Vervollständigen' 69

Abbildung 12: Abruf der Logfiles vom 18.10.2011 mittels Editor 78

Abbildung 13: Abruf der Logfiles vom 24.10.2011 mittels Editor 80

Abbildung 14: Abruf der Logfiles vom 26.10.2011 mittels Editor 82

Abbildung 15: Abruf der Logfiles vom 05.11.2011 mittels Editor 84

Abbildung 16: Logfiles vom 18.10.2011 dargestellt mittels Excel 86

Abbildung 17: Logfiles vom 24.10.2011 dargestellt mittels Excel 88

Abbildung 18: Logfiles vom 26.10.2011 dargestellt mittels Excel 90

Abbildung 19: Logfiles vom 05.11.2011 dargestellt mittels Excel 92 


\section{Abkürzungsverzeichnis}

aDIS/BMS

EDV

HEIDI

Hrsg.

ISBN

ISMN

ISSN

IT

LBZ

$M A B$

ÖB

OCLC

OPAC

$P C$

PLB

RDS

RLB

RP

RSWK

SLNP

SWD

Target-ID

UB

ZDB adaptierbares Dokumentations- und Informationssystem /

Bibliotheksmanagementsystem

Elektronische Datenverarbeitung

Heidelberger Bibliotheks-Informationssystem

Herausgeber

International Standard Book Number

International Standard Music Number

International Standard Serial Number

Informationstechnik

Landesbibliothekszentrum (Rheinland-Pfalz)

Maschinelles Austauschformat für Bibliotheken

Öffentliche Bibliothek

Online Computer Library Center

Online Public Access Catalogue

Personal Computer

Pfälzische Landesbibliothek

Resource Discovery System

Rheinische Landesbibliothek

Rheinland-Pfalz

Regeln für den Schlagwortkatalog

Simple Library Network Protocol

Schlagwortnormdatei

Target- Identifikator

Universitätsbibliothek

Zeitschriftendatenbank 


\section{Einleitung}

Seit mittlerweile knapp 30 Jahren $^{1}$ können Bibliotheksnutzer auf OPACs in Bibliotheken zurückgreifen. Seither nehmen OPACs eine immer wichtigere Rolle in Bibliotheken ein. Dies hat bereits Luis Villén-Rueda vor knapp fünf Jahren festgestellt, als er behauptete: "that the OPAC is well consolidated as an instrument for access to key information, a role continually strengthened by the growing amount of information available through electronic formats." Inzwischen wurden zahlreiche Studien über elektronische beziehungsweise Online Kataloge angefertigt. Sie sollten der Erforschung des Nutzerverhaltens dienen sowie Verbesserungsmöglichkeiten aufzeigen, denn viele Nutzer haben aus bibliothekarischer Sicht oft keine ausreichende Recherchekompetenz und sind daher auf einfache und funktionale Kataloge angewiesen. Malliari und Kyriaki-Manessi brachten dies in einem Artikel auf den Punkt: „Users do not always know what they want or are even able to explain which problem they have, what kind of difficulties they face when coming into a library or searching its online public access catalogue (OPAC)."3

Anbieter von Bibliothekssoftware erarbeiteten deswegen zahlreiche 'innovative' Verbesserungen und Weiterentwicklungen für OPACs. Auch die IT-Mitarbeiter vieler Bibliotheken entwickelten interne Lösungen für einen optimalen OPAC. Jedoch konnte bis heute noch kein 'Musterkatalog' entwickelt werden. Zu komplex sind die Strukturen der einzelnen Bibliotheken und zu unterschiedlich die Anforderungen an einen Online-Katalog. Technische und finanzielle Mittel, die den Bibliotheken für die Erwerbung für Bibliothekssoftware zur Verfügung stehen, geben oft einen nicht zu unterschätzenden Ausschlag für den Erwerb und den Umfang der Tools für einen OPAC. Hinzu kommt öfters mangelnde Prioritätensetzung zugunsten des OPACs oder fehlende Nutzersicht auf den Online-Katalog. Nicht selten erlebt man daher Nutzer, die ratlos vor einer Nulltrefferanzeige stehen oder sich plötzlich mit einer scheinbar unendlich langen Trefferliste konfrontiert sehen. Bei der Suche nach Erklärungen solcher Trefferbilder stellt sich die Frage, ob und daran anschließend, wie das in Zukunft

\footnotetext{
${ }^{1}$ Vgl. Frank, S. (2006): Gestaltung von Benutzeroberflächen und Recherchemöglichkeiten bei OPACs, S. 9

${ }^{2}$ Villén-Rueda, L, u.a.. (2007): The Use of OPAC in a Large Academic Library, S. 336

${ }^{3}$ Malliari, A. und Kyriaki-Manessi, D. (2007): Users' behaviour patterns in academic libraries' OPACs, S. 107
} 
verhindert werden könnte? Liegt es am Nutzer selbst, am OPAC oder doch an anderen Faktoren?

Dies sind spannende Fragen, die sich wohl schon viele, eingeschlossen mich selbst, bei Rechercheanfragen gestellt haben. Diese Arbeit beleuchtet Recherchen in Bibliothekskatalogen - sowohl erfolgreiche als auch negative. Hier geht es, wie es der Titel bereits verrät, um die Analyse der known-item search. Dieser Begriff stammt aus dem Englischen und bedeutet, dass nach einem bekannten Objekt gesucht wird. ${ }^{4}$ Dabei wird die Untersuchung von Logfiles an wissenschaftlichen Bibliotheken im Vordergrund stehen. Gerade bei der Suche nach etwas scheinbar Bekanntem sind Null-Treffer beziehungsweise das Vorgehen bei der Suche interessant, da man nachvollziehen kann, was der jeweilige Nutzer wollte und darauf aufbauend Rückschlüsse ziehen beziehungsweise Fehler identifizieren kann. Die Analyse in dieser Arbeit soll anhand von Logfile-Untersuchungen geschehen.

In einem Artikel von Villén-Rueda wird dies folgendermaßen beschrieben: „A transaction consists of a question put forth by the user and a response given by the system. Therefore, transactional analysis is a technique for observing user conduct and a means of reconstructing how the user interacts with the catalog, and whose final objective is to contribute to an improvement in the designs of the system." Die knownitem search entspricht einer formalen Suche, wobei es zu beachten gilt, dass nicht automatisch jede formale Suche eine known-item search ist. Hierbei wird mit formaIen Kriterien wie Autor, Titel, Erscheinungsjahr oder Verlag nach einem bekannten Medium gesucht. In der Literatur fallen meistens der Verfasser und der Titel unter die known-item search. Ein Beispiel hierfür ist bei Malliari und Kyriaki-Manessi zu finden. ${ }^{6}$ Dieser Artikel ist einer der wenigen, der sich intensiver mit der known-item search beschäftigt.

Nicht nur Gründe für das Scheitern einer Suchanfrage sollen im Folgenden herausgearbeitet werden, sondern genauso die positiven Anfragen. Die thematische Suche wird hierbei komplett ausgeklammert und nicht näher betrachtet, da dies schon in

\footnotetext{
${ }^{4}$ Vgl. Universitätsbibliothek der Humboldt-Universität zu Berlin (o.J.): Bibliotheksglossar. URL: http://www.ub.hu-berlin.de/bibliotheksglossar/known-item-search

${ }^{5}$ Villén-Rueda, L., u.a. (2007): The Use of OPAC in a Large Academic Library, S. 327

${ }^{6}$ Bsp. Malliari, A. und Kyriaki-Manessi, D. (2007): Users' behaviour patterns in academic libraries' OPACs, S. 108
} 
vielen anderen Studien geschah ${ }^{7}$ und den Rahmen dieser Arbeit sprengen würde. Aus der möglichen Datenfülle wird hierfür eine Auswahl an Logfiles der Rheinischen Landesbibliothek in Koblenz, der Pfälzischen Landesbibliothek in Speyer sowie der Bibliotheca Bipontina in Zweibrücken intensiver betrachtet, ausgewertet, analysiert und anschließend mit Logfiles der UB Heidelberg verglichen. Es sollen zudem Vergleiche und Bezüge zu ähnlichen Themen in der Literatur hergestellt werden und ein Brückenschlag in Richtung Praxis erfolgen, um die known-item search aus verschiedenen Blickwinkeln betrachten zu können.

Ziel dieser Arbeit soll es sein, neuere Erkenntnisse zur known-item search zu gewinnen und dadurch herauszufinden, was bereits gut funktioniert und welche Schwachstellen es zu verbessern gilt. Die Lösungsvorschläge sollen im Bereich der knownitem search den Nutzerkomfort bei der Recherche am OPAC steigern.

\footnotetext{
${ }^{7}$ Bsp. Weimar, A. (2004): Inhaltserschließung und OPAC
} 


\section{Aktueller Forschungsstand}

Seit dem Aufkommen von OPACs wurde dieser zu einem Objekt zahlreicher Untersuchungen. Studien und Analysen über Bibliothekssysteme gibt es schon seit dem Ende der 1960er Jahre ${ }^{8}$, also seit Beginn des EDV-Zeitalters in Bibliotheken. Auffällig ist, dass viele Studien und Ergebnisse aus den USA kommen. Ein möglicher Grund hierfür könnte ein anderes Finanzierungssystem der Bibliotheken dort sein, welches stärker auf Beiträgen und Gebühren der Nutzer basiert, wohingegen bei deutschen Bibliotheken oft noch ein 'Selbstverständnis der Existenz' vorhanden ist. Somit würde die Nutzerzufriedenheit in den USA eine viel größere Rolles spielen als hierzulande. Auch die Fülle an bibliothekswissenschaftlicher Literatur ist in den USA viel reichhaltiger als es zum Beispiel hierzulande der Fall ist.

Dieses bewusst allgemeingehaltene Kapitel soll einen Überblick über den momentanen Stand der Literatur zu OPAC-Untersuchungen ermöglichen. Zunächst werden verwendete Untersuchungsmethoden vorgestellt, dann einzelne Untersuchungsgegenstände näher betrachtet und schließlich wichtige Verbesserungsmöglichkeiten herausgearbeitet. Dies dient der Grundlage und Einordnung der in dieser Arbeit untersuchten known-item search. Bisher gibt es in der Literatur sehr wenig über eine qualitativ tiefgehende Betrachtung von Logfiles in Bezug auf die Suche nach bekannten Objekten beziehungsweise Medien in OPACs. Ein Großteil der Literatur analysiert die thematische Suche, auch als inhaltliche Suche bezeichnet. Dabei liegt der Fokus der Forschung bei Schlagwörtern, Notationen und Thesauri und es dominieren quantitative, statistisch unterlegte Untersuchungen. ${ }^{9}$

\subsection{Untersuchungsmethoden}

Im Laufe der Zeit wurden verschiedene Methoden zur Untersuchung der Nutzung des OPACs beziehungsweise zum Nutzerverhalten entwickelt und angewandt. Im Folgenden werden einige in der Literatur weitverbreitete Untersuchungsmethoden kurz dargestellt.

\footnotetext{
${ }^{8}$ Vgl. Liu, W. (2010): Understanding user OPAC searching habits through WebVoyagé Log Analysis, S. 7

${ }^{9}$ Bsp. Lau, E. P. und Goh, D. H.-L. (2006): In search of query patterns
} 
Naheliegend und in der Vergangenheit häufig angewendet ist die Benutzerbefragung. Hierzu zählen Gruppengespräche (zum Beispiel Fokusgruppen mit unterschiedlicher Recherchekompetenz) und Einzelinterviews genauso wie Erhebungen mittels Fragebögen. Diese Methoden erfordern allerdings möglichst viele Nutzer, die sich freiwillig für solche Umfragen bereiterklären müssen. Diese Umfragen, die direkt bei Nutzern durchgeführt werden, weisen oft einige Schwachpunkte auf, die nicht zu unterschätzen sind. Manfred Weichert findet die Benutzerbefragung „langwierig, aufwendig, teilweise subjektiv und in der Auswertung häufig wenig ergiebig." ${ }^{\text {10 }}$

Eine weitere Form der Nutzerbefragung ist die Thinking-Aloud-Methode. Hier werden Nutzer, meist in einer separaten Räumlichkeit, vor einen Recherche-PC gesetzt. Mittels Aufnahmegerät, Tonband oder Videokamera wird der Suchvorgang vom Nutzer selbst laut kommentiert. Diese Methode ist allerdings sehr zeitaufwendig und ebenfalls subjektiv.

Weitere häufig angewandte Methoden sind zum Beispiel Beobachtung und Protokollierung, kontrollierte Experimente, Protokollanalysen, Messungen der Effektivität bei Abfragen sowie Beurteilungen der Nutzerzufriedenheit. ${ }^{11}$

Eine Methode, bei der sich der Nutzer nicht beobachtet fühlt und sich keiner Stresssituation ausgesetzt fühlt, ist die Transaction Log Analysis oder auch Logfile-Analyse genannt. Hier werden nämlich nur die von den Nutzern im OPAC eingegebenen Daten mitgeschrieben und ausgewertet. Als Nachteil ist hier anzumerken, dass keinerlei Rückfragen an den Nutzer möglich sind. Diese Methode ist mittlerweile auf Grund verbesserter IT und Datenverarbeitungsprogramme international weit verbreitet. Näheres zu dieser Methode findet sich in Kapitel 3 'Methodische Vorgehensweise'.

Unabhängig von quantitativen Untersuchungsmethoden, die auf 'Masse' abzielen und nur stichprobenartig sind, und qualitativen Untersuchungsmethoden, die durch eine kleinere 'Masse' an auswertbarem Material tiefer in die Materie eindringen ${ }^{12}$, lässt sich feststellen, dass es keine perfekte Methode gibt und sich sowohl Vor- als

\footnotetext{
${ }^{10}$ Weichert, M. (2002): „Gibt es auch Wahnsinn“, S. 143

${ }^{11}$ Vgl. Villén-Rueda, L., u.a. (2007): The Use of OPAC in a Large Academic Library, S. 327

${ }^{12}$ Bsp. Kaske, N. K. (1993): Research Methodologies and Transaction Log Analysis
} 
auch Nachteile finden lassen. ${ }^{13}$ So wird festgestellt, dass "questionnaires, interviews and observation techniques could give user profiles, user behaviour and attitudes. At the same time, they require the researchers' presence, which could influence the results. ${ }^{14}{ }^{14}$ In vielen Fällen wird in der Forschung deshalb auf einen Mix an Analysen gesetzt.

\subsection{Untersuchungsgegenstände}

Genauso vielfältig wie die Untersuchungsmethoden selbst, sind die darin behandelten Themen, die in zahlreichen Studien vorgestellt wurden. Hier sollen nur einige wenige genannt werden, die größtenteils auf der Logfile-Analyse basieren und aus verschiedenen Ländern stammen.

Sehr häufig wurde die thematische Suche zum Objekt größerer Untersuchungen. Dieses Phänomen ist nicht nur in deutschsprachigen Quellen ${ }^{15}$, sondern auch in ausländischen Quellen ${ }^{16}$ zu finden. Oft wurde hier speziell die Recherche über Schlagwörter untersucht und die Anwendung von normiertem Vokabular wie in Deutschland zum Beispiel des RSWK- /SWD-Vokabulars in der Diplomarbeit von Alexander Weimar. ${ }^{17}$

Ebenfalls sehr aufschlussreich sind viele Untersuchungen zur Anwendung von Booleschen Operatoren UND (AND), ODER (OR), NICHT (NOT) bei einer Recherche und dem Vorgehen des Nutzers bei einer kombinierten Suche. Ausführlich wird dies zum Beispiel von Lau und Goh behandelt. ${ }^{18}$

Bereits erforscht ist das Verhältnis von einfacher und erweiterter Suche in Bibliothekskatalogen. Der Aspekt der Verteilung von Suchanfragen auf die jeweiligen Suchen und den daraus resultierenden Ergebnissen wurden ebenfalls öfters untersucht, wie zum Beispiel bei Luis Villén-Rueda nachzulesen ist. ${ }^{19}$

\footnotetext{
${ }^{13}$ Vgl. Villén-Rueda, L., u.a. (2007): The Use of OPAC in a Large Academic Library, S. 327

${ }^{14}$ Malliari, A. und Kyriaki-Manessi, D. (2007): Users' behaviour patterns in academic libraries' OPACs, S. 110

${ }^{15}$ Bsp. Vogt, R. (1999): „Es wurde keine Treffermenge gefunden“

${ }_{17}^{16}$ Bsp. Villén-Rueda, L., u.a. (2007): The Use of OPAC in a Large Academic Library

${ }^{17}$ Bsp. Weimar, A. (2004): Inhaltserschließung und OPAC

${ }^{18}$ Bsp. Lau, E. P. und Goh, D. H.-L. (2006): In search of query patterns

${ }^{19}$ Bsp. Villén-Rueda, L., u.a. (2007): The Use of OPAC in a Large Academic Library
} 
Sehr verbreitet sind zudem Analysen über die Nutzung einzelner Eingabefelder und Einschränkungen, die der Suchende während der Recherche vornimmt. Beispielsweise gehen Lau und Goh in ihrer Analyse der Frage nach, welche Eingabefelder benutzt wurden und wie Boolesche Operatoren eingesetzt wurden. ${ }^{20}$

Natürlich gibt es auch Betrachtungen eher speziellerer Themen wie zum Beispiel der Eingabe von Personennamen in die dafür vorgesehenen Felder. ${ }^{21}$

Auch das Verhalten von unterschiedlichen Nutzergruppen lässt sich über Logfiles herausfinden. Dabei wird aufgezeichnet, wer von wo aus auf den OPAC zugreift, zum Beispiel von der Bibliothek aus, vom Campus oder von außerhalb und wie zum Beispiel Lehrende oder Studierende den OPAC benutzen. Dies stellt ein Artikel von Luis Villén-Rueda anschaulich dar. ${ }^{22}$

\subsection{Forschungsüberblick}

Zuerst wird mit häufig festgestellten Ursachen bei fehlerhaften Recherchen begonnen und danach Vorschläge für Verbesserungen aufgegriffen.

Sehr weit vorne rangieren Falscheingaben seitens der Nutzer in den OPAC. Genauer betrachtet ist es vor allem fehlerhaftes Vokabular, so zum Beispiel bei der thematischen Recherche über Schlagwörter. Personennamen werden ebenfalls häufig falsch eingegeben. Hier wird entweder Vor- und Nachname in der falschen Reihenfolge als Ursache genannt, oder aber die Eingabe erfolgt in einem komplett falschen Feld. Eingaben in falsche Felder sind auch bei anderen Kategorien häufige Ursachen für Nulltrefferanzeigen.

Bei Booleschen Operatoren ist fehlendes Wissen über deren Gebrauch und Wirkung häufigste Ursache für fehlerhafte Recherchen. Dass Nutzer bei Recherchen nicht unbedingt so denken wie Bibliothekare, spiegelt sich übrigens auch bei der schon erwähnten Verwendung von normiertem Vokabular, zum Beispiel bei der thematischen Suche.

Sehr fehleranfällig sind oft auch Recherchen zur ISBN oder zu Serien. So wird statt einer ISBN öfters nach einer anderen Nummer, die auf einem Medium abgebildet ist,

\footnotetext{
${ }^{20}$ Bsp. Lau, E. P. und Goh, D. H.-L. (2006): In search of query patterns, S. $1320 \mathrm{ff}$.

${ }^{21}$ Bsp. Weichert, M. (2002): „Gibt es auch Wahnsinn“, S. 146 f.

22 Bsp. Villén-Rueda, L., u.a. (2007): The Use of OPAC in a Large Academic Library
} 
recherchiert. ${ }^{23}$ Dies ist auch einer der wenigen Artikel, im dem explizit die known-item search erwähnt wird. Hier wird sie verglichen mit der thematischen Suche.

Desweiteren sind auch Suchstrategien, die Trunkierungen verwenden, oftmals fehleranfällig.

Ursula Schulz geht nach ihren Auswertungen sogar soweit, daraus eine 'Ignoranz' der Nutzer gegenüber Trunkierungsmöglichkeiten und Thesauri abzuleiten, ohne Nachdenken würde alles eingegeben. ${ }^{24}$

Hinweise auf andere häufige Fehler, wie zum Beispiel Schreibfehler und das Vertippen bei der Eingabe, sind überall in der Literatur zu finden.

Lösungsansätze werden aufgezeigt und beschrieben. So setzt Manfred Weichert, wie viele andere auch, auf Nutzerschulungen. Jedoch nennt er im gleichen Zug ein groBes Problem von Nutzerschulungen, nämlich die nachlassende Zahl an interessierten Teilnehmern. ${ }^{25}$

Sehr häufig anzutreffen sind auch pauschale Aussagen zur Verbesserung des OPAC, zur übersichtlicheren Darstellung der Suchoberfläche oder pauschal zur stärkeren Ausrichtung an Nutzerbedürfnissen. ${ }^{26}$ Konkrete Vorschläge, wie dies im Einzelnen zu erreichen sei, werden aber nicht immer genannt. Zu erkennen ist dies zum Beispiel bei Renate Vogt, die zwar eine ausführliche Analyse der Recherche am OPAC vornimmt, aber keine Verbesserungsvorschläge bietet. ${ }^{27}$

Praktische Tipps haben sehr häufig automatische Wortformreduktionen, automatische Rechtschreibkorrektur und semantisch-syntaktische Nachbearbeitungsprogramme zum Inhalt. Diese technischen Aspekte werden zum Beispiel sehr detailliert von René Schneider beschrieben. ${ }^{28}$

Weitere häufig genannte Lösungsmöglichkeiten sind beispielsweise die Verbesserung der Hilfe im OPAC. ${ }^{29}$ Kürzere Hilfetexte, verständlichere Formulierungen oder besseres Auffinden der Hilfeanzeige etwa sind hierbei Schritte in die richtige Richtung. Auch Vorschläge für Verbesserungen von normiertem Vokabular für die thematische Suche werden gemacht. Man solle dieses Vokabular mehr an die Sprache der

\footnotetext{
${ }^{23}$ Bsp. Malliari, A. und Kyriaki-Manessi, D. (2007): Users' behaviour patterns in academic libraries' OPACs

${ }^{24}$ Vgl. Schulz, U. (1994): Was wir über den OPAC-Nutzer wissen, S. 299

${ }^{25}$ Vgl. Weichert, M. (2002): „Gibt es auch Wahnsinn“, S. 149

${ }^{26}$ Bsp. Weimar, A. (2004): Inhaltserschließung und OPAC

${ }^{27}$ Bsp. Vogt, R. (1999): „Es wurde keine Treffermenge gefunden“

${ }^{28}$ Bsp. Schneider, R. (2009): OPACs, Benutzer und das Web

${ }^{29}$ Vgl. Dreßler, J. (2004): Suche in Bibliothekskatalogen, S. 92
} 
Nutzer anpassen, anstatt bibliothekarische Fachsprache zu verwenden. Ein weiterer Lösungsvorschlag besteht darin, die gesamte Systematik natürlichsprachlich darzustellen, meint zumindest Alexander Weimar in seiner Diplomarbeit. ${ }^{30}$ Auch eine Reduktion der Suchmodi, wie sie René Schneider vorschlägt ${ }^{31}$, wird als mögliche Lösung gegen Nulltrefferanzeigen in Erwägung gezogen.

Welche Ergebnisse aus den in dieser Arbeit vorliegenden Logfiles gewonnen werden können und welche Verbesserungen bei Nulltrefferanzeigen und anderen fehlerhaften Recherchen hierfür vorgeschlagen werden, wird im Kapitel 5 (Nutzerwissen und Verbesserungsmöglichkeiten) ausführlich dargelegt.

${ }^{30}$ Vgl. Weimar, A. (2004): Inhaltserschließung und OPAC, S. 44

${ }^{31}$ Vgl. Schneider, R. (2009): OPACs, Benutzer und das Web, S. 17 


\section{Methodische Vorgehensweise}

Für die vorliegende Arbeit wurde eine Auswahl aus 21.315 Logfiles näher betrachtet und untersucht. Als Vergleich diente eine Auswahl aus circa 100.000 Logfiles eines anderen OPACs. Logfiles, auch Log-Dateien genannt, sind Dateien, mit denen Prozesse, die in Computern und Netzwerken ablaufen, aufgezeichnet werden. ${ }^{32}$ Das System protokolliert hierbei im Hintergrund automatisch die Eingaben der Nutzer mit. Kurth merkt dazu an, dass die Wahrnehmung der Nutzer nicht gespiegelt wird, es keine Rückschlüsse über Emotionen der Suchenden gibt und auch nichts über deren Hintergrundwissen bekannt ist. ${ }^{33}$ Die Logfiles sind also nur Rohdaten, die jede Menge Raum für Interpretationen lassen, aber dafür auch nicht von äußeren Einflüssen verfälscht sind.

Zur Menge der ausgewerteten Logfiles muss angemerkt werden, dass nur die known-item search näher betrachtet wurde und die thematische Suche keine Beachtung fand. Die qualitative Untersuchung basiert auf der Auswertung tiefergehender Analysen einer relativ geringen Anzahl Logfiles. In den Kapiteln 4 (Logfile Analyse) und 5 (Nutzerwissen und Verbesserungsmöglichkeiten) werden Ergebnisse und daraus resultierende Verbesserungsvorschläge diskutiert.

\subsection{Herkunft der Logdateien}

Die in dieser Arbeit untersuchten Logfiles stammen von der Pfälzischen Landesbibliothek in Speyer (PLB), der Rheinischen Landesbibliothek in Koblenz (RLB) und der Bibliotheca Bipontina in Zweibrücken. Mitgeloggt wurden 21.315 Logfiles im Zeitraum vom 18.10.2011 bis zum 24.10.2011. Die drei Bibliotheken sind Teil des Landesbibliothekszentrum Rheinland-Pfalz (LBZ), zu welchem auch noch die Büchereistellen in Koblenz und Neustadt/Weinstraße gehören.

Die PLB fühlt sich als Landesbibliothek des ehemaligen Regierungsbezirks Pfalz den folgenden fünf Leitlinien verpflichtet:

\footnotetext{
${ }^{32}$ Vgl. IT Wissen (o.J.): Das große Online-Lexikon für Informationstechnologie. URL: http://www.itwissen.info/definition/lexikon/logfile-Log-Datei.html

${ }^{33}$ Vgl. Kurth, M. (1993): The limits and limitation of transaction log analysis
} 
1. „Versorgung der Bevölkerung, der Kultur- und Bildungseinrichtungen, der Wirtschaftsbetriebe und Behörden mit Medien und Informationen.

2. Sammlung, Erschließung, Bewahrung und Vermittlung der Rheinland-PfalzLiteratur für den ehem. Regierungsbezirk Pfalz und Mitarbeit an der Erstellung der Landesbibliographie, Pflichtexemplarbibliothek für den ehemaligen Regierungsbezirk Pfalz.

3. Pflichtexemplarbibliothek für die Amtsdruckschriften des Landes RheinlandPfalz

4. Bewahrung historischer Medienbestände in Rheinland-Pfalz

5. Schwerpunkte innerhalb des LBZ:

Musiksammlung

Historischer Buchbestand, Restaurierung “ ${ }^{34}$

Hierfür hält die Bibliothek einen Bestand von etwas mehr als einer Million Medien, darunter mehr als 4.000 laufende Zeitschriften, für die interessierte Öffentlichkeit bereit. $^{35}$

Die RLB bietet ihren Nutzern einen Gesamtbestand von knapp 600.000 Medien, mit ca. 3.000 laufenden Zeitschriften, an. ${ }^{36}$ Zu ihren Aufgaben zählt sie folgende Punkte:

1. „Versorgung der Bevölkerung, der Kultur- und Bildungseinrichtungen, der Wirtschaftsbetriebe und Behörden mit Medien und Informationen

2. Sammlung, Erschließung, Bewahrung und Vermittlung der Rheinland-PfalzLiteratur für den ehem. Regierungsbezirk Koblenz (einschließlich Digitalisierung) und Erstellung der Landesbibliographie, Pflichtexemplarbibliothek für den ehemaligen Regierungsbezirk Koblenz

3. Pflichtexemplarbibliothek für die Amtsdruckschriften des Landes RheinlandPfalz

4. Bewahrung historischer Medienbestände in Rheinland-Pfalz

5. Funktion einer Pädagogischen Zentralbibliothek für das Land RheinlandPfalz ${ }^{\text {“37 }}$

\footnotetext{
${ }^{34}$ Landesbibliothekszentrum Rheinland-Pfalz (2011): Pfälzische Landesbibliothek. URL: http://www.lbz-rlp.de/cms/plb/ueber-uns/daten-fakten/index.html

${ }^{35} \mathrm{Vgl}$. ebd.

${ }^{36}$ Landesbibliothekszentrum Rheinland-Pfalz (2011): Rheinische Landesbibliothek. URL: http://www.lbz-rlp.de/cms/rlb/ueber-uns/daten-fakten/index.html

${ }^{37}$ Ebd.
} 
Das LBZ Rheinland-Pfalz verwendet für seine wissenschaftlichen Bibliotheken, eingeschlossen beide Landesbibliotheken, einen SISIS-webOPAC. Dieses Modul stammt von SISIS-SunRise und wird von OCLC vertrieben. Der Katalog bietet seinen Nutzern die Möglichkeit der Recherche und Bestellung in den Beständen der Pfälzischen und Rheinischen Landesbibliothek sowie der Bibliotheca Bipontina in einem Katalog. Zudem gibt es personalisiertes Arbeiten mit einmaliger Anmeldung für alle Vorgänge innerhalb einer Sitzung und dauerhaftes Speichern der Sucheinstellungen und -ergebnisse. Außerdem besteht die Möglichkeit, ältere Suchanfragen aufzurufen oder diese mit einer neuen Suchanfrage zu kombinieren.

Der Katalog verzeichnet Bücher und Zeitschriften, aber auch Noten, Karten, audiovisuelle Medien und vieles mehr, aber keine Aufsätze.

Es existiert nur eine Suchmaske, die allerdings auch eine freie Suche, gleichzusetzen mit einer einfachen Suche, beinhaltet (siehe Abb. 1).

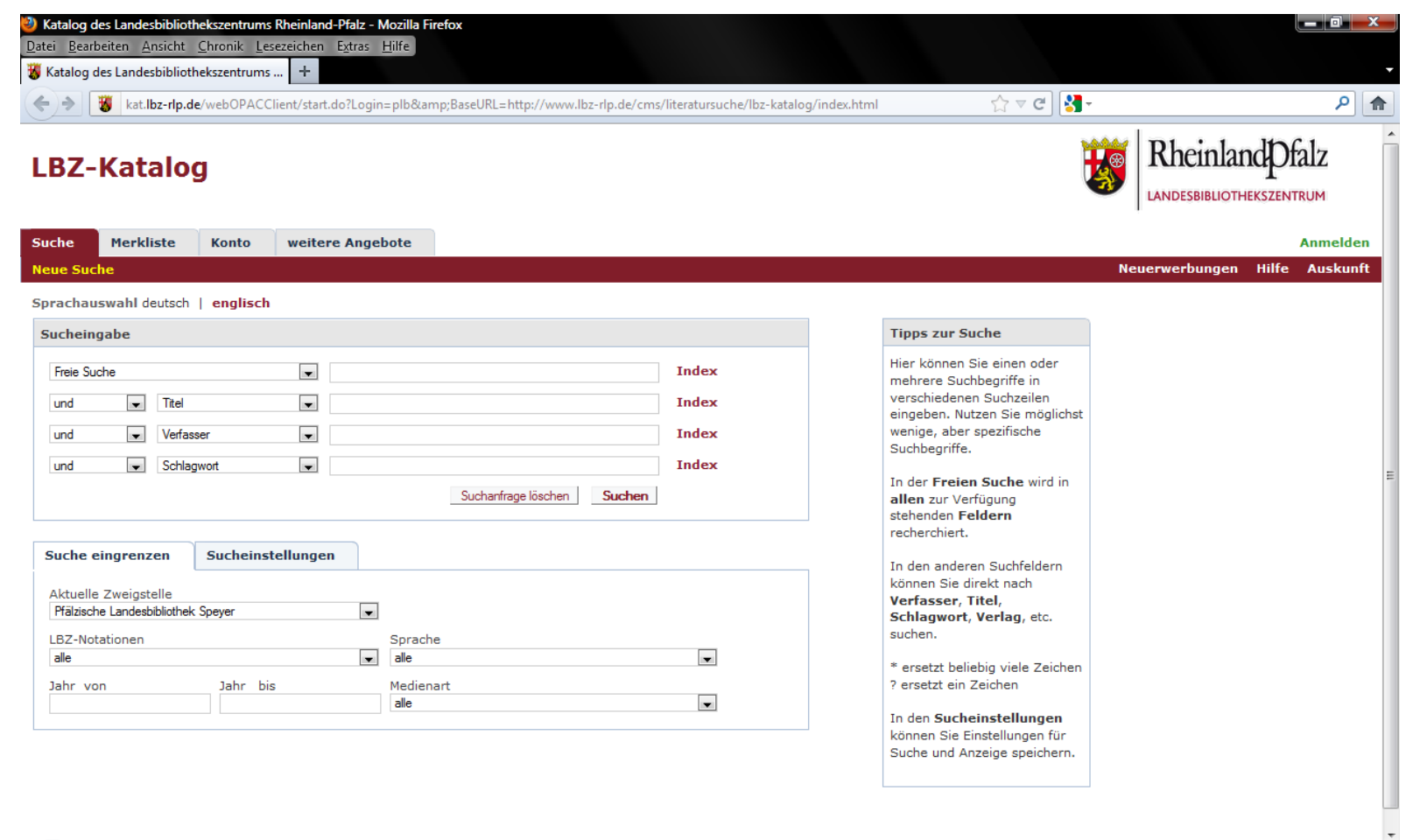

Abb. 1: Suchmaske des LBZ-Katalogs

Es lässt sich darüber hinaus über 14 Suchschlüssel wie Verfasser, Titel, Musik oder Schlagwort suchen. Die Suchanfragen können über Boolesche Operatoren UND, ODER, UND NICHT verknüpft werden. Mit diesen Operatoren kann auch eine Verknüpfung zu einer vorhergehenden Suchanfrage hergestellt werden. 
Eine weitere Eingrenzung ist zum Beispiel über die Auswahl der Zweigstelle oder der Medienart möglich.

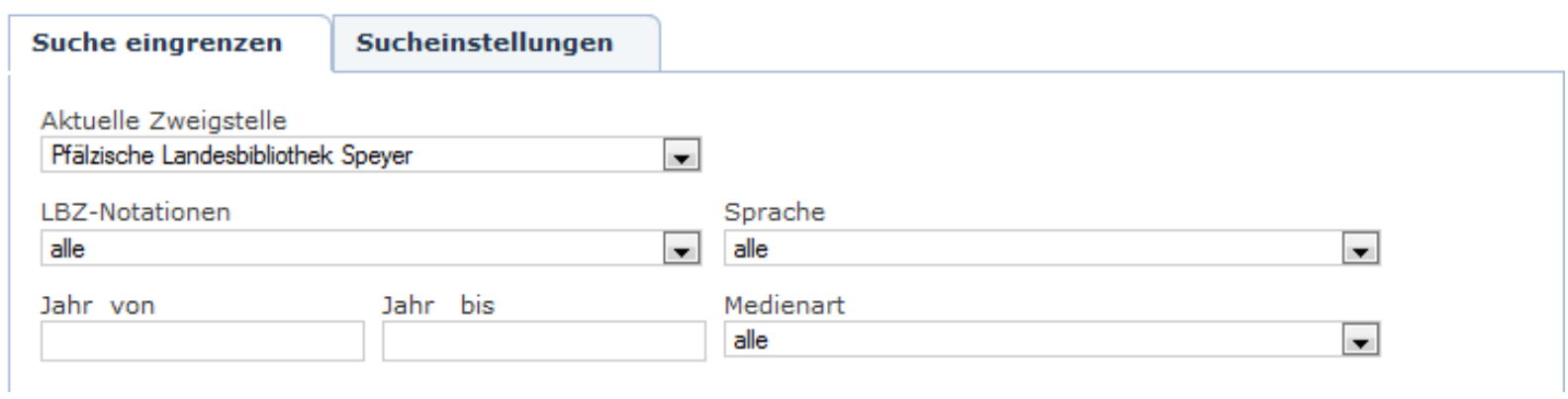

Abb. 2: LBZ-Katalog: Eingrenzung der Suche

Sucheinstellungen wie der Eintrag in eine Merkliste oder die Sortierung der Trefferliste nach bestimmten Kriterien lassen sich ebenfalls zusätzlich auswählen.

\begin{tabular}{|l|l|l|}
\hline Suche eingrenzen & Sucheinstellungen & \\
\hline Sortierung/Exemplare & & \\
\hline Sortierung nach Zweigstelle & & max. Trefferanzahl pro Seite \\
Eintrag in Merkliste & 10 \\
\hline Temporare Merkliste & 20 \\
\hline
\end{tabular}

Abb. 3: LBZ-Katalog: Sucheinstellungen

Es bestehen die Möglichkeiten von Rechtstrunkierungen, Linkstrunkierungen, und Mitteltrunkierungen mittels Platzhalter ‘*‘, welcher mehrere alfanumerische Zeichen ersetzen kann. Für die Ersetzung eines einzigen alfanumerischen Zeichens wird der Platzhalter '?' verwendet.

Es sind im LBZ-Katalog noch Reiter für die Merkliste, das Konto und weitere Angebote (Internet-Links/ Zusatzfunktionen) zu finden. Verlinkt wird darüber hinaus zu Neuerwerbungen, zur Hilfe und zur Auskunft.

Kurz wird hier auf die Logfiles eingegangen, die als Vergleichsdaten in dieser Arbeit herangezogen wurden. Sie stammen von der UB der Ruprecht-Karls-Universität Heidelberg und wurden vom 18.10.2011 bis zum 05.11.2011 mitgeloggt.

Die UB Heidelberg dient in erster Linie der Literaturversorgung aller Universitätsangehörigen der Universität Heidelberg. Hierfür hält sie circa sechs Millionen Bände bereit. Hiervon sind allerdings nur 4,5 Millionen Titel im OPAC nachgewiesen. 
Medien ab dem Erscheinungsjahr 1973 sind vollständig verzeichnet. Bestände der dezentralen Bibliotheken sind erst ab 1990 vollständig im OPAC erfasst.

Es wird ein selbstentwickelter Katalog verwendet. Die Kommunikation mit dem Bibliotheksinformationssystem SunRise (OCLC) erfolgt über das SLNP-Protokoll von OCLC (Ausleihstatus, Benutzerselbstbedienungsfunktionen etc.). So sind in HEIDI, so der Name des Heidelberger OPACs, viele Funktionen und Eigenschaften, die sich bei anderen OPACs als positiv oder nützlich herausgestellt haben, übernommen worden. Zusätzlich erfolgte eine Anpassung in Richtung Suchmaschinentechnologie. Beispielhaft ist hier die Rechtschreibkorrektur oder die Autovervollständigung zu nennen ${ }^{38} \mathrm{HEIDI}$ ist speziell auf das Heidelberger Bibliothekssystem zugeschnitten. HEIDI bietet wie die meisten Bibliothekskataloge eine einfache Suche und eine erweiterte Suche.

Die einfache Suche besteht aus einem Suchfeld, in dem verschiedene Kategorien wie zum Beispiel Autor, Erscheinungsjahr, ISBN und anderes mehr eingegeben werden können.

Die erweiterte Suche bietet drei Suchfelder, die über ein Pull-Down Menü 22 Suchoptionen, wie zum Beispiel Schlagwort, Körperschaft oder Medienart, zur Recherche zulassen. Die Suchfelder lassen sich durch Boolesche Operatoren wie im Katalog LBZ Rheinland-Pfalz verknüpfen. Auch die Trunkierung ist in diesem Opac mit der der LBZ identisch. In der Suchhistorie sind vorhergehende Rechercheanfragen gespeichert und wieder abrufbar. Es besteht, im Gegensatz zum LBZ-OPAC, die Möglichkeit, mittels der über der Trefferliste stehenden einfachen Suche ohne umständliche Navigation, eine neue Suche zu starten. Sowohl die einfache Suche wie auch die erweiterte Suche bieten Optionen wie der Auswahl von Teilkatalogen oder Anzeigeoptionen der Ergebnisse, um die Recherche einzugrenzen. Die angezeigten Treffer lassen sich nochmals einschränken durch die Auswahl weiterer Kategorien wie Jahreszahlen, Sprache oder Datenträger.

\footnotetext{
${ }^{38}$ Vgl. Maylein, L. (2010): Dauerbaustelle OPAC? URL: http://www.vdb-online.org/veranstaltung/522/maylein_heidi.pdf
} 


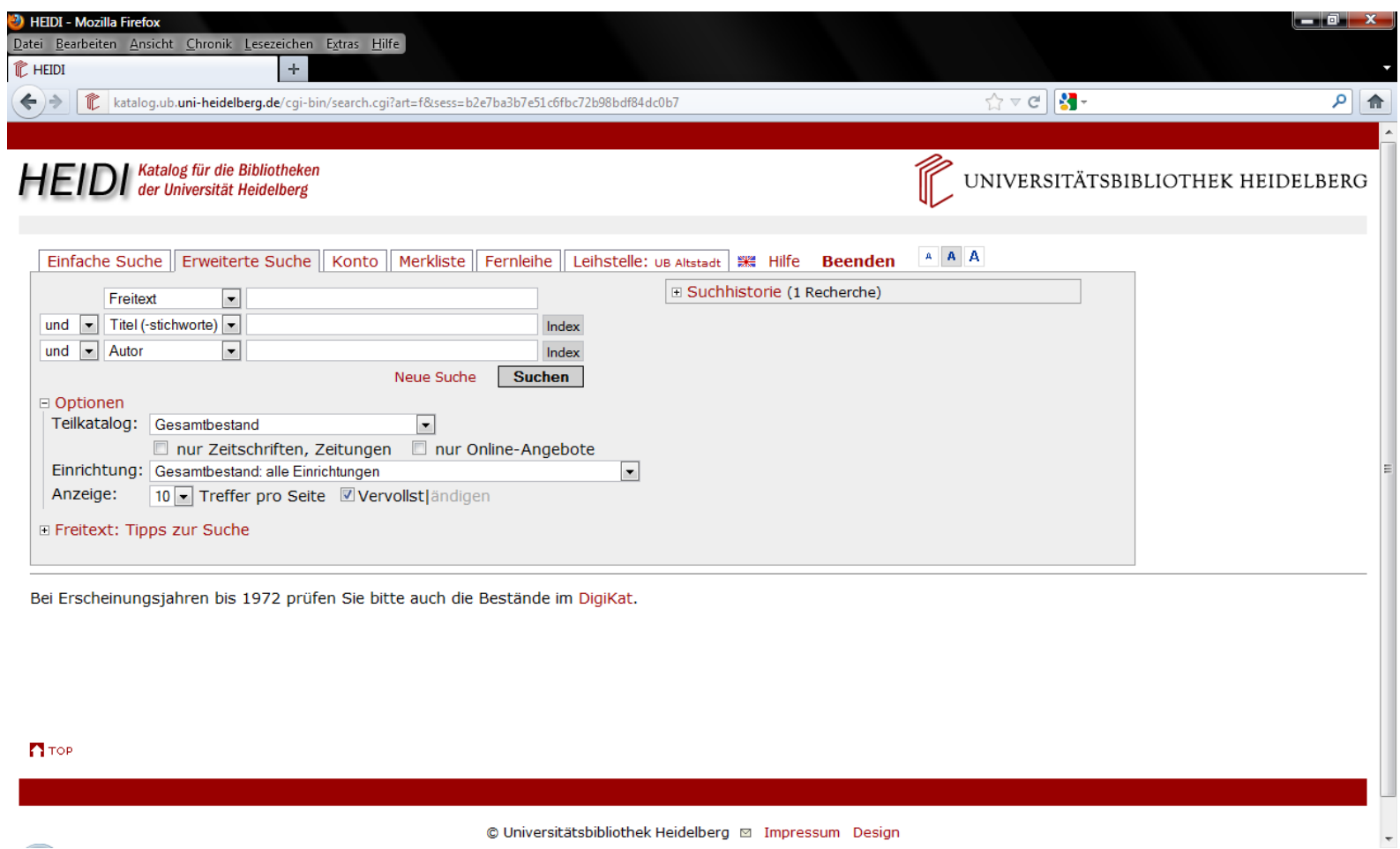

Abb. 4: Die erweiterte Suche in HEIDI

In Heidi lassen sich noch die Reiter Konto, Merkliste, Fernleihe und Leihstelle finden. Ein Link über 'Hilfe' führt Informationen über den OPAC und die Recherchemöglichkeiten auf.

\subsection{Inhalte der Logfiles}

Eine Log-Datei kann entweder sehr viele unterschiedliche Informationen speichern oder aber nur sehr wenige Informationen enthalten. Hierfür gibt es keinerlei Normierungen. Generell gilt, je mehr Informationen vorliegen, desto umfangreicher werden die Interpretation und die Auswertung der Daten.

Der Umfang der Daten hängt natürlich vom verwendeten Bibliothekssystem ab und der Schnittstelle, die für einen Export der Daten zur Verfügung steht.

Die Logfiles des LBZ (Anhang A.1a / A.1b) beinhalten folgende relevante Datenfelder:

- Ort der Suche (PLB, RLB oder Bibliotheca Bipontina)

- Datum und Uhrzeit der Recherche 
- Trefferanzahl

- einfache Suche oder erweiterte Suche

- Zeit, die eine Abfrage benötigt

- Suchkategorie als MAB-Kategorie und Suchbegriff beziehungsweise Suchbegriffe

Logfiles der UB Heidelberg (Anhang A.1c / A.1d) beinhalten:

- eine Sessiongrenze, also eine Zuordnung von Recherchen zu einem Benutzer

- die Trefferanzahl einer Anfrage

- die Suchkategorie in normierter Form (z.B. 'au' für Autor) und den Suchbegriff beziehungsweise Suchbegriffe

- vorgenommene Einschränkungen bei Kurztitelanzeigen (z.B. Materialart)

Nur anhand dieser zwei verschiedenen Logfiles erkennt man schon einen Unterschied bezüglich des Informationsgehalts und deren mögliche Inhalte. Diese beiden Logfiles mögen sich noch einigermaßen gleichen, es kann aber auch weit größere Unterschiede bei Logfiles geben.

Die UB Tübingen zum Beispiel konnte auf Grund eines Systemwechsels für die vorliegende Arbeit zum jetzigen Zeitpunkt keine Logfiles im eigentlichen Sinne liefern. Für aDIS/BMS konnte das Protokoll nur in einer Datenbanktabelle abgelegt werden, nicht aber in einer Dateiform. Das Problem dabei ist eine fehlende Standardschnittstelle und fehlende Erfahrungswerte mit dem neuen System. So können zum Beispiel nur die Eingabe, einige Suchaspekte und das Datum mitgeloggt werden. Informationen über Trefferanzahl und Datum fehlen komplett. ${ }^{39}$

Die Logfiles der in der Literatur angestellten Untersuchungen und Analysen beinhalteten in den meisten Fällen Informationen über Rechercheanfragen, Trefferanzahl und die Auswahl einfache Suche oder erweiterte Suche. ${ }^{40}$ Hier gibt es natürlich noch einiges mehr, jedoch sind Logfiles immer bibliotheksabhängig und werden erstellt mit der primären Fragestellung: Wozu werden die Daten benötigt? Bei ausländischen Systemen können Logfiles stark variieren. Dies hängt sicherlich auch mit den Datenschutzbestimmungen der einzelnen Länder zusammen.

\footnotetext{
${ }^{39}$ Vgl. Fuchs, H.-J. (2010): Mail vom 28.11.2011

${ }^{40}$ Bsp. Lau, E. P. und Goh, D. H.-L. (2006): In search of query patterns
} 


\subsection{Umwandlung der Logfiles in Klarschrift}

Das vorhergehende Kapitel skizzierte die Untersuchungsgegenstände. Um diese aus den Logfiles gewinnen zu können, müssen die Rohdaten umgewandelt werden. Diese Rohdaten sind meist in Dateien mit kryptischen Endungen wie zum Beispiel '.201011' enthalten. Öffnen lassen sich diese Dateien mit einem gewöhnlichen Texteditor wie 'Notepad++'. Jede Datei enthält mehrere tausend Zeilen mit Recherchedaten des OPACs, die wie in diesem Beispiel, nach dem Zeitpunkt des Suchvorgangs sortiert sind:

rlb\% \%20.10.11-00:20:31\%-1\%35\%2\%43\%100="würtz"

rlb\% \%20.10.11-00:22:20\%-1\%90\%2\%43\%100="wirtz"

Diese beiden Zeilen sind in der Reihenfolge: 'OPAC-Kennung, Datum/Uhrzeit, Target-ID, Trefferanzahl, Suchmodus, Suchzeit in Millisekunden und Suchbegriff' aufgebaut. Die Probleme bei dieser Art der Darstellung liegen auf der Hand, denn die Daten:

1. sind unübersichtlich und schlecht lesbar

2. lassen sich nicht sortieren

3. sind über mehrere Dateien verteilt und nicht zusammengefasst

Als Lösung dieser Probleme wurde für diese Arbeit ein Java-Programm benutzt, das die Dateien einliest und in strukturierter Form als Exceltabelle ausgibt (Anhang B.1a B.1d). Hierfür wurde folgendermaßen vorgegangen:

1. Logdatei öffnen, leere Exceldatei vorbereiten (Überschriften einfügen).

2. Eine Zeile der Logdatei einlesen (entspricht einem Eintrag).

3. Wenn die Zeile nicht leer ist, so wird sie anhand eines Delimeters, in diesem Fall das '\%', zerteilt. Dadurch erhält man einzelne Datensätze, die man in die Zellen der Exceldatei schreiben kann.

4. Datensätze identifizieren (nach fester vorgegebener Reihenfolge) und in eine Zeile in die Exceldatei schreiben. Jedes Datenelement bekommt eine eigene 
Spaltennummer $\mathrm{Y}$, die aktuelle Zeilennummer ist $\mathrm{X}$. Somit kann gezielt im Koordinatensystem der Exceldatei die Zelle $[\mathrm{X}, \mathrm{Y}]$ beschrieben werden.

5. Wenn die nächste Zeile leer ist und das Ende der Datei erreicht ist, wird die Exceldatei gespeichert und alle Dateien beendet.

Dieser Vorgang kann in einem Durchgang für beliebig viele Logdateien erfolgen und liefert je eine Exceltabelle. Durch minimale Anpassung können auch mehrere Logdateien auf einmal eingelesen und in eine einzige Exceltabelle geschrieben werden. Hierbei ist jedoch zu beachten, wie viele Zeilen pro Tabelle von der jeweils eingesetzten Excelversion unterstützt werden. Die Exceltabellen können nun zum Beispiel nach Trefferzahl oder Suchzeit sortiert werden. Dies erlaubt schnellere Rückschlüsse auf Zusammenhänge mit dem jeweiligen Suchbegriff. 


\section{Logfile-Analyse}

Die folgende Auswertung basiert auf den bereits erwähnten Logdaten der PLB, RLB und der Bibliotheca Bipontina. Die Logfiles der drei Bibliotheken sind nicht getrennt mitgeloggt worden und so findet auch in der Untersuchung keinerlei Unterschied zwischen diesen Bibliotheken statt. Insgesamt 21.315 Logfiles, mitgeloggt in einem Zeitraum von sieben Tagen, wurden hierfür in Auswahl ausgewertet. Für jeden Tag existiert eine eigene Datei bzw. Exceltabelle.

Statistisch wurden die Logfiles jedes einzelnen Tages im Zeitraum von 9.00 Uhr bis 9.59 Uhr ausgewertet. Daraus ergab sich eine Anzahl von ca. 1.400 Logfiles. Zudem fiel die Zeit von 15.00 Uhr bis 15.59 Uhr unter eine grobe Suche nach Besonderheiten, wie auffällige Recherchemuster oder Null-Treffer.

Insgesamt wurden somit etwa 3.000 Logfiles näher auf Besonderheiten und Auffälligkeiten betrachtet. Da die nachfolgenden Zahlen auf einer manuellen Auszählung basieren, können diese nur Nährungswerte sein. Da Logfiles außerdem in einigen Fällen einen Interpretationsspielraum zulassen, kann nicht immer eindeutig geklärt werden, ob der betreffende Recherchevorgang einer thematischen Suche oder einer known-item search entspricht.

\subsection{Recherchevorgehen}

Sehr auffällig ist die starke Tendenz der Recherche nach bekannten Objekten, also eine known-item search. Insgesamt stehen 959 known-item searches 441 thematischen Suchen gegenüber. Prozentual gesehen ergibt dies eine Verteilung von rund $68 \%$ für die Suche nach bekannten Objekten und 32\% für Suchen über ein Thema. Wird der Fokus wie angekündigt auf die known-item search gelegt, so lassen sich die im Folgenden beschriebenen Vorgehensweisen finden.

Die einfache Suche wurde nur 310-mal benutzt, wohingegen die erweiterte Suche 596-mal verwendet wurde. Eine Kombination von einfacher und erweiterter Suche fand rund 30-mal statt. Diese ungleiche Verteilung mag mehrere Gründe haben. Die einfache Suche als einfacher Suchschlitz ist lediglich auf der Homepage des LBZ 
Rheinland-Pfalz unter den jeweiligen Bibliotheken wie 'Pfälzische Landesbibliothek' zu finden. Wie viele Nutzer auf diese Seiten gelangen oder dieses Item wahrnehmen ist nicht feststellbar.

\begin{tabular}{|c|c|c|}
\hline $\begin{array}{l}\text { Landes Bibliotheks } \\
\text { ÜBER Uns }\end{array}$ & & \\
\hline SERVICE & & \\
\hline LITERATUR SUCHE & 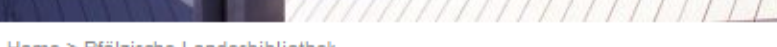 & \\
\hline LESEFÖRDERUNG & Home P Fralzische Landesbibliothex & Schnelleinstieg \\
\hline LANDESKUNDE & Landesbibliothekszentrum / Pfälzische & \\
\hline BIBLIOTHECA BIPONTINA & Landesbibliothek & Diqitale Bibliothek \\
\hline $\begin{array}{l}\text { PFÄLZISCHE } \\
\text { LANDE SBIB LIOTHEK }\end{array}$ & $\begin{array}{l}\text { Otto-Mayer-Str. } 9 \\
67346 \text { Speyer }\end{array}$ & 니Texpress \\
\hline Über uns & Telefon: $062329006-224$ & $\begin{array}{l}\text { Rheinland-Ptalzische } \\
\text { Bibliographie }\end{array}$ \\
\hline Service & $\begin{array}{l}\text { Telefax: } 062329006-270 \\
\text { E-Mail: info.plb@lbz-rlp.de }\end{array}$ & dilibri \\
\hline Fernleihe und mehr & & Handschriften- und \\
\hline Digitale Angebote & Öffnungszeiten: & $\overline{\text { Nachlasskatalog }}$ \\
\hline Veranstaltungen & Montag bis Freitag 9 bis $18 \mathrm{Uhr}$ & Aktuelles \\
\hline Führungen / Schulungen & Samstag 9 bis 12 Uhr & \\
\hline $\begin{array}{l}\text { RHEINISCHE } \\
\text { LANDESBIBLIOTHEK }\end{array}$ & & $\begin{array}{l}\text { Stellenangebot (mehr) } \\
\text { Veranstaltungen } 2012\end{array}$ \\
\hline BÜCHEREISTELLE KOBLENZ & Suche im LBZ-Katalog & Bestandsliste zu Hugo \\
\hline BÜCHEREISTELLE NEU STADT & 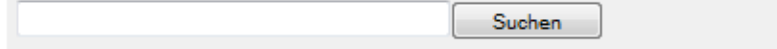 & Ball \\
\hline $\begin{array}{l}\text { Kontakt } \\
\text { Anfahrt } \\
\text { Öffnungszeiten } \\
\text { Impressum }\end{array}$ & 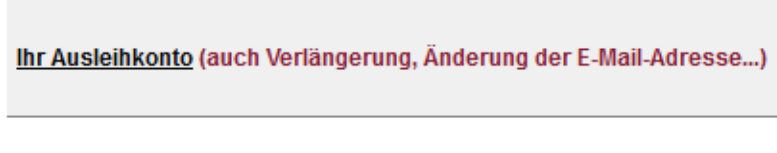 & $\begin{array}{l}\text { Bestandsliste zum } \\
\text { Thema "Salier" } \\
\text { f Facebook } \\
\text { RSS-Feed }\end{array}$ \\
\hline
\end{tabular}

Abb. 5: LBZ-Katalog: Einfache Suche auf der Seite des Landesbibliothekszentrums / Pfälzische Landesbibliothek

Wahrscheinlicher ist es, dass viele Nutzer über 'Literatursuche' und 'LBZ-Katalog' eine Bibliothek auswählen und deswegen direkt auf den OPAC geleitet werden. 


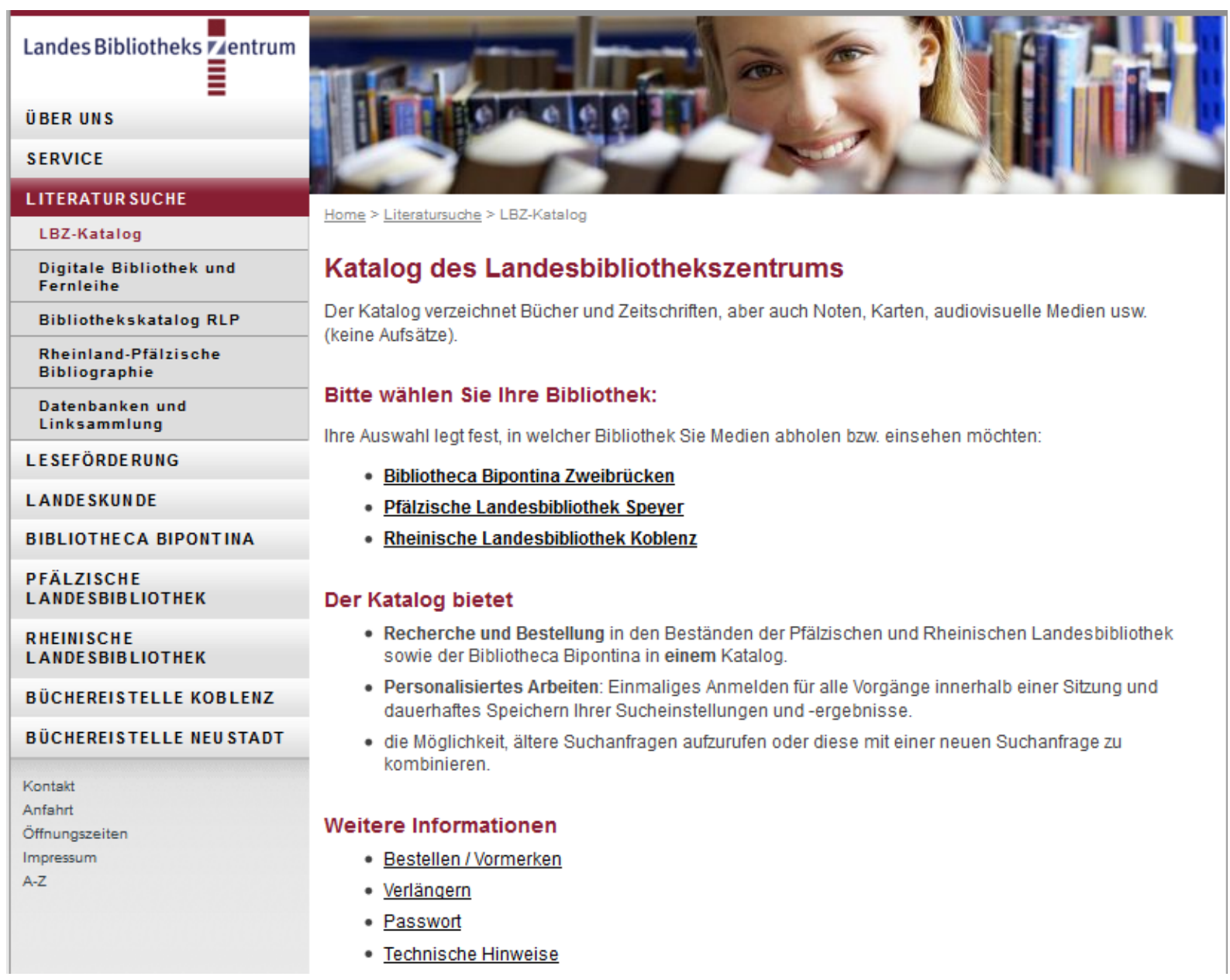

Abb. 6: LBZ-Katalog: Direkter Weg zum OPAC

Im OPAC selbst gibt es keinen einfachen Suchschlitz, sondern eine einzige Maske mit der Auswahl sämtlicher Recherchemodi.

Das Feld freie Suche im OPAC, auch als Freitextsuche bezeichnet, zählt ebenfalls zur einfachen Suche.

\begin{tabular}{|l|l|l|l|l|}
\hline Sucheingabe & Index \\
\hline und & Index & \\
\hline
\end{tabular}

Abb. 7: LBZ-Katalog: Suchfenster mit freier Suche 
Dennoch nutzen viele Kunden laut Auswertung häufiger die für ihre Bedürfnisse passenden Suchkategorien. Dies könnte an den bereits erwähnten, etwas versteckten Feldern der einfachen Suche liegen. Änderungsmöglichkeiten hierzu sind in Kapitel 5.2 'Verbesserungsmöglichkeiten der Recherche' zu finden.

Die starke Nutzung der erweiterten Suche kann aber auch an der wissenschaftlichen Klientel der Landesbibliotheken beziehungsweise der Bibliotheca Bipontina liegen. Die Nutzer mögen hier recherchekompetenter sein als beispielsweise Nutzer einer ÖB oder Studenten des Grundstudiums einer UB. Möglicherweise sind viele Nutzer auch etwas ältere Personen, die die Google-Mentalität noch nicht verinnerlicht haben und es gewöhnt sind, mittels Datenbanken zu recherchieren.

Bei 70\% der betrachteten known-item searches wurde nur eine Suchoption verwendet. Spitzenreiter ist hierbei der 'Titel'. Insgesamt 37\% der Recherchen erfolgten über diese Einstellung.

Ebenfalls weit vorne und an zweiter Stelle ist die Suche nach dem 'Verfasser'. 21\% der Suchenden nutzten diese Suchoption.

Kaum ins Gewicht fallen Recherchen nach 'Signaturen' beziehungsweise 'Standardnummern'. Diese Kategorien verteilen sich auf Recherchen zu 9\% nach einer 'Signatur', und nur 3\% der Recherchen beziehen sich auf eine 'ISBN/ISSN/ISMN'.

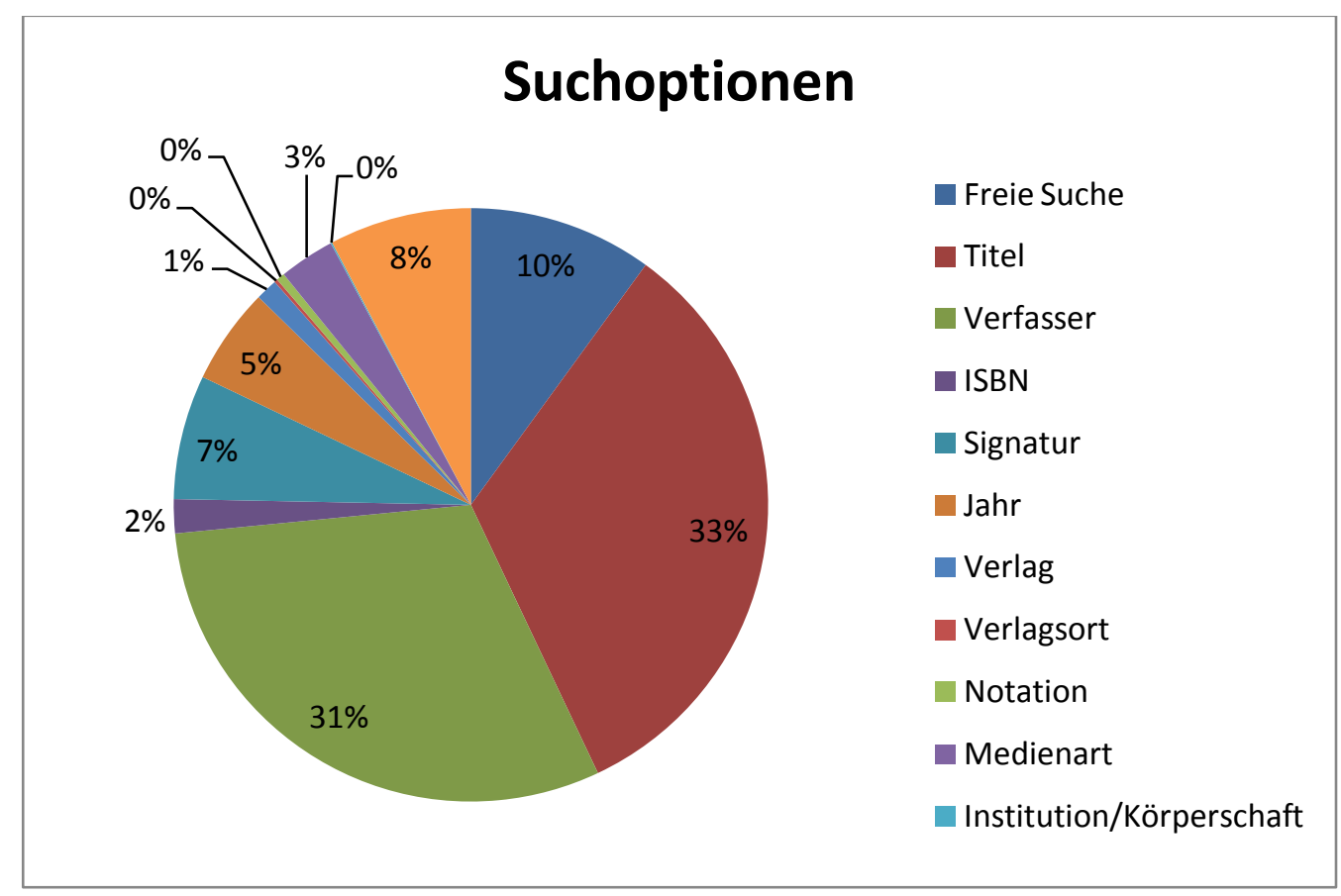

Abb. 8: Prozentuale Verteilung der Suchoptionen in der erweiterten Suche. Die known-item search (Titel / Verfasser / ISBN / Signatur) erzielt insgesamt 73\%. 
Verknüpft mit dem Booleschen Operator UND wurden insgesamt 32\% der Recherchen.

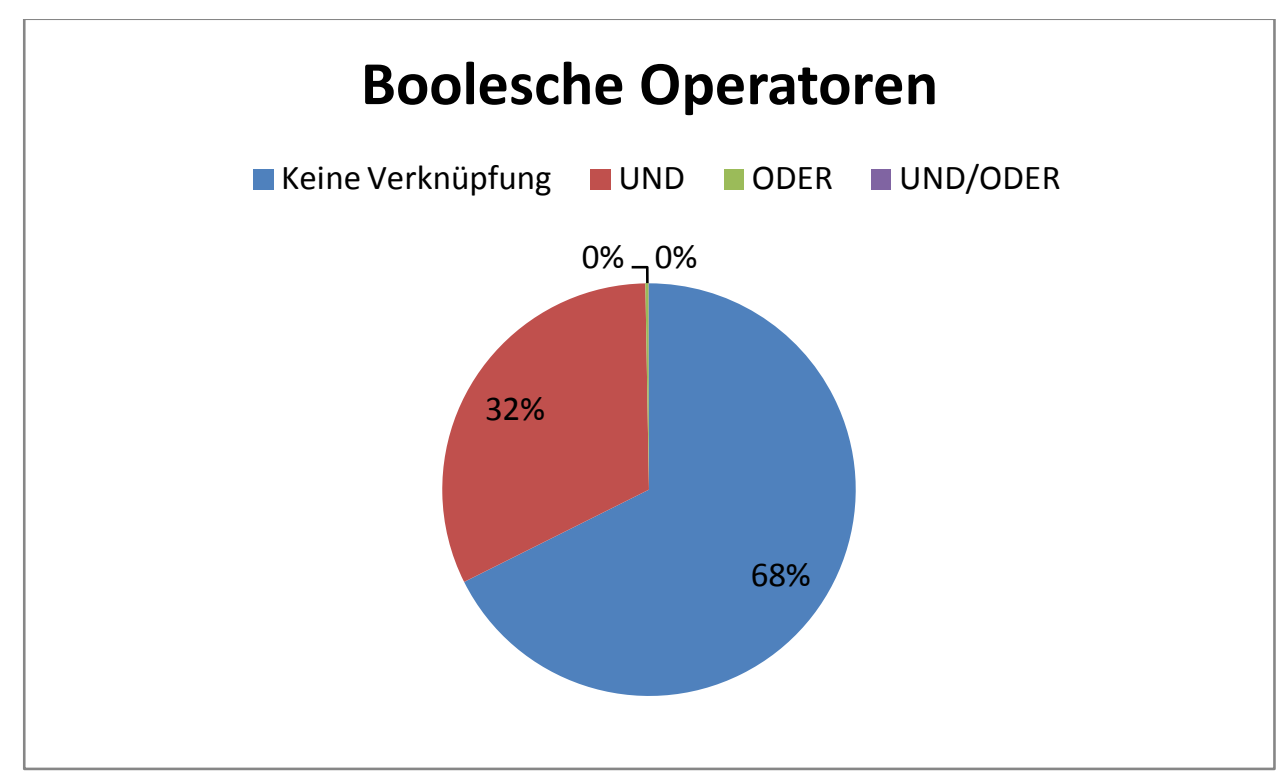

Abb. 9: Prozentuale Darstellung der Booleschen Operatoren bei der erweiterten Suche

Diese verteilen sich auf Recherchen zu 18\% mit einer Kombination aus 'Titel' und 'Verfasser' und $12 \%$ sind sonstige Kombinationen, bei denen etwa Sucheinstellungen aus 'Verfasser' und 'Jahr' abgefragt wurden.

Diese Angaben decken sich mit Feststellungen aus der Literatur. Der Artikel von Malliari und Kyriaki-Manessi weist folgende Zahlen aus: Es entfallen $41 \%$ der Recherchen auf den 'Titel' und 27\% der Recherchen auf den 'Verfasser'. ${ }^{41}$ Abweichende Werte sind bei Manfred Weichert zu finden. Hier finden knapp 50\% der Recherchen über den Titel statt und 23\% über den Verfasser. ${ }^{42}$

Bei 29\% der known-item searches erfolgten Null-Treffer, was angesichts der Ausgewerteten Menge von 959 Logfiles etwas mehr als ein Viertel der Recherchen ausmacht.

In der Literatur sind zum Beispiel bei René Schneider ähnliche Zahlenwerte in Bezug auf Null-Treffer zu finden. ${ }^{43}$

\footnotetext{
${ }^{41}$ Vgl. Malliari, A. und Kyriaki-Manessi, D. (2007): Users' behaviour patterns in academic libraries' OPACs, S. 115

${ }^{42}$ Vgl. Weichert, M. (2002): „Gibt es auch Wahnsinn“, S. 144

${ }^{43}$ Vgl. Schneider, R. (2009): OPACs, Benutzer und das Web, S. 13
} 
Mehrheitlich, nämlich 49\% der Treffer, ergibt eine Trefferliste von unter zehn Anzeigen. Sehr oft wurde sogar nur ein einziger Treffer erzielt.

Bei 15\% der Recherchen erfolgte eine Kurztitelanzeige mit bis zu 50 Titeln. Marginal sind Kurztitelanzeigen mit bis zu 100 Treffern, nämlich 3\%, und mit mehr als 100 Treffern (4\%).

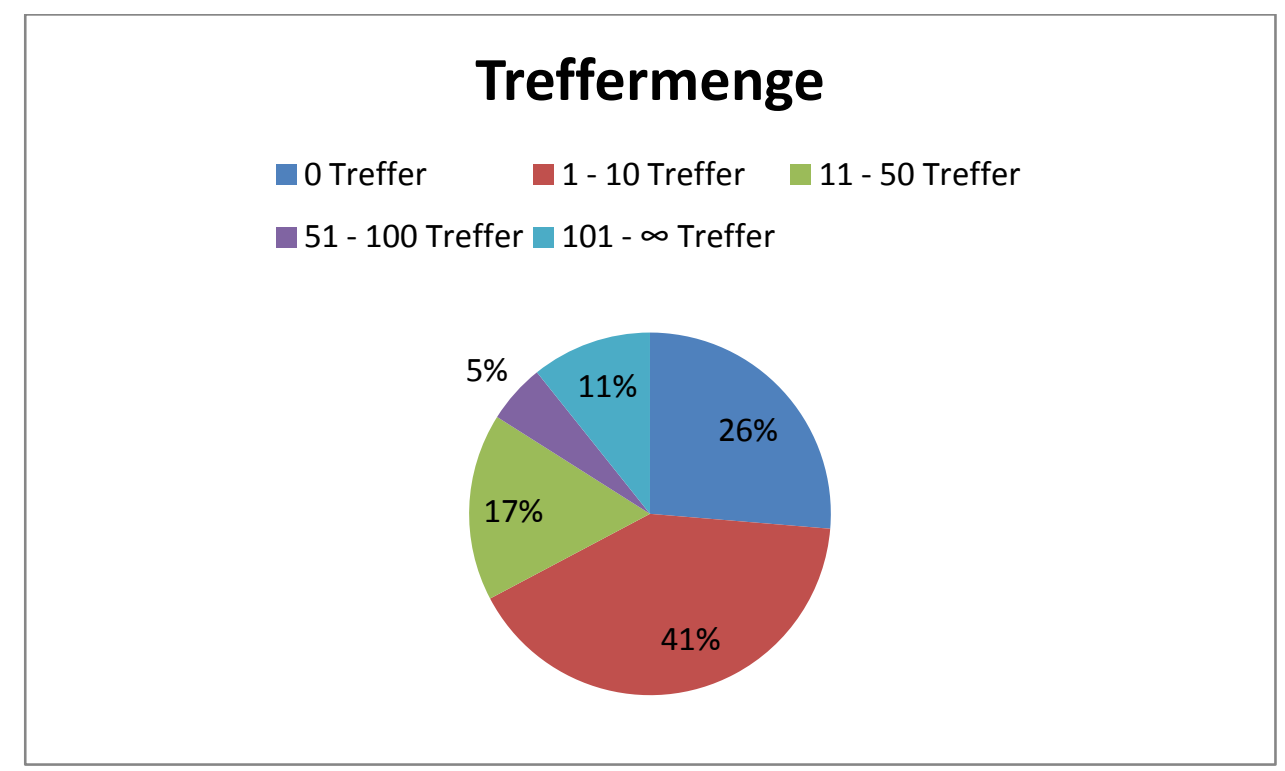

Abb. 10: Prozentuale Darstellung der Treffermenge

\subsection{Auffällige Verhaltensmuster bei der Recherche}

Mehrere Auffälligkeiten sind bei der Recherche zu known-item searches zu finden. In diesem Kapitel werden diese Verhaltensmuster zunächst beschrieben, Gründe für Auffälligkeiten und Verbesserungsvorschläge werden im nachfolgenden Kapitel 5 (Nutzerwissen und Verbesserungsmöglichkeiten) näher beleuchtet.

Sehr erstaunlich ist die hohe Zahl an Nutzern, die nur mit dem Nachnamen eines innen bekannten Verfassers suchen. So ist es nicht verwunderlich, dass gerade bei häufig verbreiteten Familiennamen sehr viele Treffer erzielt werden. Für einige Nutzer sind die ausgegebenen Ergebnisse schon zu viele, um sie alle zu überprüfen. So wird zum Beispiel selbst bei zwölf Treffern (Bsp. 4.2.1) eine erneute Recherche vorgenommen. Dies erklärt sich daraus, dass die meisten dieser Nutzer sofort den Vornamen zu einer erneuten Recherche hinzunahmen. In den meisten Fällen schränkte 
sich dadurch das Ergebnis deutlich ein. Sind unter einer Person immer noch viele Werke angezeigt, kombinierten die Suchenden den Verfasser zum Beispiel mit Titelstichwörtern. In der Regel führte dies dann zu einem gewünschten Treffer.

Beispiele aus der Praxis:

Bsp. 4.2.1

Einfache Suche

"mitschang"

Recherche mittels Nachnamen in der einfachen Suche erbrachte zwölf Treffer.

Einfache Suche

"theo mitschang"

Die Recherche mit derselben Suchoption, aber mit Vorname, ergab nur noch einen Treffer.

\section{Bsp. 4.2.2}

Erweiterte Suche: Verfasser

"swift"

Verwendung des Nachnamens bei der Recherche ergaben 81 Treffer.

Erweiterte Suche: Titel \& Verfasser

"letzte Runde" \& "swift"

Bei einem weiteren Rechercheanlauf, diesmal mit Hinzunahme des Titels, wurde genau ein Treffer aufgezeigt. 


\title{
Bsp. 4.2.3
}

Einfache Suche

"Berthold"

Die Recherche mit der einfachen Suche nach einem Nachnamen erbrachte 2197 Treffer. Die Vielzahl der Treffer kann auch dadurch bedingt sein, dass Vornamen ebenfalls in der Trefferliste erscheinen.

\author{
Einfache Suche \\ "Berthold Erika"
}

Eine erneute Recherche mittels Vor- und Zuname ergab noch zehn Treffer.

Erweiterte Suche: Verfasser \& Titel \& Verlagsort

"Erika Berthold" \& "Erzieherinnen sind doof" \& "Neuwied"

Durch Verwendung der erweiterten Suche und der Angabe des Verlagsortes konnte das Ergebnis auf zwei passende Treffer eingeschränkt werden.

Oftmals wird bei einer Recherche nach dem Titel dieser komplett, vermutlich durch copy and paste, mit sämtlichen Füll- und Stoppwörtern eingegeben. Dasselbe gilt für einen Artikel am Anfang des Hauptsachtitels, der häufig bei der Recherche mit eingegeben wird. 
Beispiele aus der Praxis:

\section{Bsp. 4.2.4}

Erweiterte Suche: Titel

"Die deutsche Literatur. Ein Abriss in Text und Darstellung: Naturalismus"

Diese Anfrage, in der der Suchende wohl alles eingegeben hat, was er wusste, erbrachte keinen Treffer.

Erweiterte Suche: Titel

"Die deutsche Literatur Naturalismus"

Durch ein deutliches Verkürzen der Anfrage konnte die Treffermenge auf acht Treffer erhöht werden. Der Grund für eine Nulltrefferanzeige lag daran, dass 'Die deutsche Literatur. Ein Abriss in Text und Darstellung' der übergeordnete Titel eines Sammelbandes und 'Naturalismus' der Titel eines Teilbandes ist.

\section{Bsp. 4.2.5}

Einfache Suche

"Nah und doch so fern"

Diese Titelsuche mittels der einfachen Suche ergab 30 Treffer.

\section{Erweiterte Suche: Titel}

"Nah und doch so fern"

Durch Eingabe derselben Suchanfrage in der Erweiterten Suche konnte die Treffermenge auf 20 Treffer reduziert werden. Der passende Titel ist jedoch nicht im LBZ Rheinland-Pfalz zu finden. 


\section{Bsp. 4.2.6}

\section{Einfache Suche}

"Business Model Management: Design - Instruments - Success Factors, Wiesbaden 2011"

Diese Recherche war nicht erfolgreich, da die suchende Person die englische Ausgabe des Buches gesucht hat. Die Suche nach dem deutschsprachigen Werk mit dem Titel „Business Model Management: Design - Instrumente - Erfolgsfaktoren, Wiesbaden 2011“ hätte hingegen den gewünschten Treffer erzielt.

Die Suche nach einem Verfasser wie auch die Suche nach einem Titel bieten folgende interessante Verhaltensmuster. So wird bei der Recherche in der einfachen Suche oftmals ein Minimalaufwand an Eingabe betrieben. Dies führt nicht selten zu sehr langen Trefferlisten. Meistens wird in diesem Fall in die erweiterte Suche gewechselt, um dort in den entsprechenden Feldern weiterzusuchen. Das Ergebnis ist hierbei qualitativ deutlich besser als im vorhergehenden Recherchevorgang.

Beispiele aus der Praxis:

\section{Bsp. 4.2.7}

Einfache Suche

"die frau in der"

Diese Suchanfrage, das Fragment eines Titels, erbrachte in der einfachen Suche 7181 Treffer.

Einfache Suche

"die frau in der hinteren" 
Bei einer erneuten Rechercheanfrage wurde das Titelfragment minimal erweitert. Dies führte allerdings schon zu einem passenden Treffer.

\title{
Bsp. 4.2.8
}

\section{Einfache Suche \\ "Prengel"}

Hier erfolge die Suche nach einem Verfasser mittels Nachnamen in der freien Suche und lieferte sieben Treffer.

\section{Erweiterte Suche: Verfasser}

"Prengel"

Durch eine Eingabe des Nachnamens in die dafür vorgesehene Suchoption in der erweiterten Suche reduzierte sich die Treffermenge auf fünf Treffer.

\author{
Erweiterte Suche: Verfasser \\ "Prengel, Annedore"
}

Durch die Hinzunahme des Vornamens in derselben Suchoption konnte die Trefferanzahl allerdings nicht weiter minimiert werden.

\section{Bsp. 4.2.9}

Einfache Suche

"Organisationsmanagement" 
Auf den ersten Blick sieht dies wie eine thematische Suche aus, da nur ein einziges aussagekräftiges Wort in der einfachen Suche eingegeben wurde. Erzielt hat diese Anfrage 119 Treffer.

Einfache Suche \& Erweiterte Suche: Verfasser

"Organisations-Management" \& "Bokranz"

Jedoch hat eine weitere Rechercheanfrage, mit Verfasser, gezeigt, dass der Suchende einen bestimmten Titel wollte. Diese Anfrage hatte nur noch einen Treffer zur Folge.

Die meisten Nutzer geben in der Regel bei einer Nulltrefferanzeige nicht sofort auf, sondern starten eine erneute Recherche. In vielen Fällen ist diese erfolgreicher und führt zum gewünschten Medium.

Auslöser einer negativen Rechercheanfrage sind häufig Rechtschreibfehler. Je länger eine Eingabe ist, desto größer ist auch die Gefahr von fehlerhaften Wörtern oder Zeichen. In sehr vielen Fällen erfolgt meist sofort die Korrektur des begangenen Fehlers.

Beispiele aus der Praxis:

Bsp. 4.2.10

Erweiterte Suche: Titel

"Hqndbuch qualitative Entwicklungspsychologie"

Hier war der Titel dem Suchenden bekannt, aber ein Rechtschreibfehler verhinderte eine erfolgreiche Recherche. 
Erweiterte Suche: Titel

"Handbuch qualitative Entwicklungspsychologie"

Durch die Korrektur des Fehlers konnte in einem erneuten Anlauf das passende Medium gefunden werden.

Eingaben in falsche Suchmodi und falsch angewendete Kombinationen führen ebenfalls öfters zu einem negativen Ergebnis. Nutzer nehmen hier Korrekturen vor, die auf eine erneute, aber oftmals verkürzte Eingabe oder einer Eingabe in weniger Suchfelder abzielt.

Beispiele aus der Praxis:

Bsp. 4.2.11

Erweiterte Suche: Titel \& Verfasser \& Verlagsort

"Handbuch Vorstellungsgespräch" \& "Hesse, Jürgen; Schrader, Hans Christian" \& "Frankfurt am Main, Eichborn"

Diese Anfrage weist gleich mehrere Fehler auf. So wurden zwei Verfasser, statt aufgeteilt auf zwei Suchfelder, in ein Suchfeld geschrieben. Ebenso wurde der Verlagsnamen, statt in ein eigenes Suchfeld, in die Suchoption für den Verlagsort hineingeschrieben.

Erweiterte Suche: Titel \& Verfasser

"Handbuch Vorstellungsgespräch" \& "Hesse Jürgen"

Durch diese Anfrage erhielt der Suchende dann den passenden Treffer. 
Die sogenannte Schiller-Räuber-Problematik tritt gelegentlich auf und führt zu Nulltreffern. Bei diesem Problem enthält sowohl der Datensatz der übergeordneten Einheit Informationen aus der Suchanfrage, wie auch der Datensatz eines Einzelbandes. Um Redundanzen zu vermeiden werden Eintragungen aus dem übergeordneten Datensatz nicht mehr wiederholt.

Beispiele aus der Praxis zur Illustration:

Bsp. 4.2.12

Erweiterte Suche: Verlag \& Jahr \& Verfasser \& Schlagwort "Winkler" \& "1988" \& "Gerard de Nerval" \& "Lorelei"

Diese Recherche, bestehend aus mehreren Kombinationen, war nicht erfolgreich. Die Angaben des Nutzers waren dennoch nicht falsch, sondern konnten in dieser Kombination nicht das gewünschte Ergebnis liefern. Das gesuchte Buch ist ein beigefügtes Werk eines Teilwerkes einer Reihe. Zum besseren Verständnis sind folgend die Anzeigen aus dem OPAC des LBZ übernommen worden:

Übergeordneter Titel:

Verfasser: $\quad$ Nerval, Gérard $\neg$ de

Hrsg./Bearb.: $\quad$ Miller, Norbert [ᄀHrsg.]

TITEL: Werke

Verfasserang.: [hrsg. von Norbert Miller ...]

Verlagsort: München

Verlag: Winkler

Reihe: Winkler-Weltliteratur-Dünndruck-Ausgabe

Schrift/Sprache: Aus d. Franz. übers.

LBZ-Notation: $\quad 250$ 


\section{Einzelband:}

Band: 2
T I T E L:
Die Oktobernächte
Jahr:
1988
Umfangsangabe: $623 \mathrm{~S}$. : III.
ISBN:
3-538-05339-1
ISBN:
3-538-05839-3
Beigefügtes Werk: Lorelei [u.a.]
LBZ-Notation:
250
$\rightarrow$ das gesuchte Werk

Äußerst fehleranfällig, zumindest was die Eingabe betrifft, sind bei der known-item search Signaturen. Auf die Recherche mittels Signatur wird öfters zurückgegriffen. Diese Suchanfragen sind jedoch nicht immer von Erfolg gekrönt. Oftmals erhalten Nutzer eine Nulltrefferanzeige bei dieser Recherchemethode. Fast immer liegt dies an falsch eingegebenen Bestandteilen einer Signatur oder hat seine Ursache darin, dass Bestandteile der Signatur schlicht weggelassen wurden.

Beispiele aus der Praxis:

Bsp. 4.2.13

Erweiterte Suche: Signatur

"DVD789"

Die Eingabe der Signatur erfolgte hier ohne Leerzeichen und ergab folglich keinen Treffer.

Erweiterte Suche: Signatur

"DVD 789"

Eine erneute Anfrage, dieses Mal mit Leerzeichen eingegeben, führte zum passenden Medium. 


\section{Bsp. 4.2.14}

Erweiterte Suche: Signatur

"111-42921"

Diese Anfrage nach einer Signatur ergab keinen Treffer, da die Signaturen in der LBZ Rheinland-Pfalz dreistellig Strich vierstellig (XXX-YYYY) sind.

\section{Bsp. 4.2.15}

Erweiterte Suche: Signatur

"Per. 16497*"

Die Trunkierung einer Signatur ist zwar nicht sehr häufig zu finden, kann aber mit höherer Wahrscheinlichkeit zum gewünschten Medium führen, auch wenn es wie in diesem Fall elf Treffer zur Folge hat.

Die Eingabe einer ISBN kann ebenso fehleranfällig sein wie die Eingabe einer Signatur. Jedoch führen Recherchen mittels ISBN bei der vorliegenden Analyse mehrheitlich zum Erfolg. Die ISBN-Recherche wird allerdings höchst selten angewandt.

Beispiele aus der Praxis:

\section{Bsp. 4.2.16}

Erweiterte Suche: ISBN

"9783189900781"

Ein Beispiel für eine falsche ISBN. Die Eingabe von Bindestrichen ist für die Recherche nicht relevant. 


\section{Bsp. 4.2.17}

Erweiterte Suche: ISBN

"978-3834423313"

Auch diese ISBN wurde nicht korrekt wiedergegeben.

Bsp. 4.2.18

Erweiterte Suche: ISBN

"978-3-8371-0395-3"

Diese ISBN, sogar mit Bindestrichen eingegeben, führte in diesem Fall zu dem gesuchten Medium.

Viele Nutzer recherchieren vor allem nach einer erfolglosen Recherche oder einer Recherche mit zu vielen Treffern mittels Kombinationen verschiedener Suchmodi. Am beliebtesten sind Kombinationen aus Titel und Verfasser. Diese Kombination führt die Nutzer in der Regel auf dem schnellsten Weg zum gewünschten Ergebnis. Jedoch wird auch häufig aus einer Kombination mit Verfasser und einer Jahresangabe gesucht. Seltener werden eher außergewöhnliche Kombinationen verwendet. So lassen sich z.B. Recherchen mit den Modi Titel und Verlag oder Titel und Körperschaft finden.

Beispiele aus der Praxis:

\section{Bsp. 4.2.19}

Erweiterte Suche: Titel

"propaganda drittes reich" 
Mit dieser Titelanfrage hat der Nutzer kein zufriedenstellendes Ergebnis erzielt.

Erweiterte Suche: Titel \& Verfasser

"propaganda" \& "thymian bussemer"

Durch die Kombination mit einem Verfasser und der Verkürzung des Titels konnte das richtige Buch (Titel: Propaganda und Populärkultur: konstruierte Erlebniswelten im Nationalsozialismus) gefunden werden.

Bsp. 4.2.20

Einfache Suche

"einführung pädagogische psychologie"

Die Recherche in der freien Suche nach diesem Titel erbrachte 79 Treffer.

Einfache Suche

"einführung pädagogische psychologie rosemann bielski"

Die gleiche Recherche, diesmal aber mit Verfasser, konnte dem Suchenden das gewünschte Ergebnis, einen Treffer, liefern.

\section{Bsp. 4.2.21}

Erweiterte Suche: Titel \& Verlag

"tinto" \& "cornelsen"

Hier wurde erfolgreich mit einer Kombination aus Titel und Verlag recherchiert. 
Nicht nur die eben erwähnten Verknüpfungen mittels des Booleschen Operators UND lassen sich in den Logfiles finden, sondern auch Trunkierungen. Diese treten im Gegensatz zu den Booleschen Operatoren seltener auf. Am Auffälligsten ist eine Trunkierung bei der Materialart. Hier wird auffällig oft die Materialbenennung 'Zeitschrift' als 'Zeit*' trunkiert, was allerdings dem System geschuldet ist. Bei den eingrenzenden Optionen wird die Medienart 'Zeitschrift/Zeitung' automatisch trunkiert.

Beispiele aus der Praxis:

\section{Bsp. 4.2.22}

Erweiterte Suche: Titel \& Medienart

"Grundschulmagazin" \& "Zeit*"

Hier wurde offensichtlich eine Zeitschrift gesucht. Der Suchende verwendete hierfür den Titel der Zeitschrift in Kombination mit der Medienart, die das System automatisch trunkiert. Dies sind die häufigsten auftretenden Trunkierungen.

\section{Bsp. 4.2.23}

Erweiterte Suche: Verfasser

"hensen, g"

Diese Rechercheanfrage blieb erfolglos, da mit einem abgekürztem Vornamen gesucht wurde. Ob der Nutzer diesen trunkieren wollte oder nicht lässt sich im Nachhinein nicht mehr feststellen. Jedoch ist es im LBZ Rheinlang-Pfalz OPAC erst ab drei Buchstaben möglich zu trunkieren. So hätte der Nutzer hier gar keine Chance gehabt dies zu tun.

Erweiterte Suche: Verfasser

"hensen" 
Eine folgende Suche mit dem Nachnamen erbrachte eine Trefferliste von 17 Titelanzeigen.

Bsp. 4.2.24

Freie Suche \& Erweiterte Suche: Titel

"dreharbeit*" \& "Freihand"

Diese Recherche, die allerdings auch eine thematische Suche sein könnte, erzielte keinen Treffer. Es ist völlig unklar, was der Suchende damit bezwecken wollte. Wusste er, was er wollte und hat aus Unsicherheit beziehungsweise Bequemlichkeit trunkiert, oder hat er auf 'gut Glück' etwas gesucht?

Eine 'Kapitulation' auf Grund einer Nulltrefferanzeige kommt für die allermeisten Nutzer nicht in Frage. Es werden entweder zwei oder auch drei Anläufe unternommen, um auf das gewünschte Medium zu stoßen.

Aber auch mehr als drei Rechercheanfragen kommen vor. So hat zum Beispiel ein Nutzer insgesamt rund 34 Anfragen abgeschickt, aber dennoch kein zufriedenstellendes Ergebnis erhalten. Öfters sind die gewünschten Medien schlichtweg nicht im Bestand enthalten.

Beispiele aus der Praxis:

\section{Bsp. 4.2.25}

Erweiterte Suche: Verfasser

"daniele"

Einfache Suche

"darmstadt daniele" 
Erweiterte Suche: Titel \& Medienart

"Grundschulmagazin" \& "Zeit*"

Einfache Suche

"Kooperation von Anfang an"

Erweiterte Suche: Medienart

"Grundschulmagazin"

Einfache Suche

"grundschulmagazin"

Einfache Suche

"Grundschulmagazin 74"

Erweiterte Suche: Titel

"grundschulmagazin 74"

Erweiterte Suche: Titel

"Grundschulmagazin"

Erweiterte Suche: Titel

"grundschulmagazin"

Erweiterte Suche: Titel

"kooperation von anfang an"

Erweiterte Suche: Titel \& Verfasser

"Grundschulmagazin" \& "Darmstadt daniele"

Einfache Suche \& Erweiterte Suche: Medienart

"Kooperation von anfang an" \& "Zeit"

Erweiterte Suche: Verfasser \& Medienart

"Daniele Darmstadt" \& "Zeit" 
Erweiterte Suche: Titel

"Kooperation von Anfang an. Erziehungspartnerschaft im Netzwerk"

Einfache Suche \& Erweiterte Suche: Titel \& Jahr

"Daniele" \& "Kooperation von Anfang an" \& "2006"

Einfache Suche \& Erweiterte Suche: Titel \& Jahr

"Daniele" \& "Grundschulmagazin" \& "2006"

Erweiterte Suche: Medienart \& Titel

"Zeitschriftenaufsatz" \& "Kooperation von Anfang an."

Erweiterte Suche: Medienart \& Titel \& Jahr

"Zeitschrift" \& "Grundschulmagazin 74" \& "2006"

Dies sind Auszüge aus insgesamt circa 23 Rechercheanfragen die ein Nutzer getätigt hat, um einen Artikel aus einer Zeitschrift zu finden. Gesucht wurde hierbei unter anderem mit der Verfasserin des Artikels, dem Titel der Zeitschrift, dem Titel des Aufsatzes und mit Jahreszahlen. Die Zeitschrift, und somit auch der Artikel, sind im Bestand des LBZ RP. Allerdings wurde, wie allgemein üblich im LBZ RP, der einzelne Aufsatz nicht im OPAC erfasst. So blieb die Recherche, obwohl die Zeitschrift angezeigt wurde, schlussendlich erfolglos. Eine Recherche in Google zeigt folgendes Ergebnis:

'Daniele Darmstadt/Hedi Plän/Jürge Stapelmann: Kooperation von Anfang an. Erziehungspartnerschaft im Netzwerk von Kindertagesstätten und Grundschule‘.

\section{Bsp. 4.2.26}

Erweiterte Suche: Titel \& Verfasser

"Welt verstehen - Wirklichkeit konstruieren" \& "Fischer, E. (hrsg.)" 
Erweiterte Suche: Verlag \& Jahr \& Titel

"borgmann" \& "2004" \& "Welt verstehen - Wirklichkeit konstruieren" \& "Fischer, E. (hrsg.)"

Erweiterte Suche: Verlag \& Verfasser

"borgmann" \& "Fischer, E."

Erweiterte Suche: Verlag \& Verfasser

"borgmann" \& "Fischer, Erhard"

Auch dieser Nutzer hat mehrere Anläufe unternommen, um sein Buch zu finden. Dabei hat er wohl alles eingegeben was er darüber wusste. Nur die letzte Anfrage konnte inm schließlich einen Treffer liefern.

\section{Bsp. 4.2.27}

Erweiterte Suche: Titel \& Verfasser

"spezielle zoologie teil 2" \& "westheide"

Erweiterte Suche: Titel \& Verfasser

"spezielle zoologie" \& "rieger"

Erweiterte Suche: Titel \& Verfasser

"spezielle zoologie" \& "westheide"

Dieses Werk besteht aus mehreren Bänden, von denen nicht alle Herausgeber bei der Katalogisierung berücksichtigt wurden. Außerdem ist zum Titel die Bandbezeichnung hinzugefügt worden. Dies sind zwei Gründe, die zum Scheitern beitrugen. Diese Recherche, mit circa drei Suchvorgängen, ist ein Fall, der bei der Logfileuntersuchung häufiger auftrat. Das heißt, dass Nutzer bei einem nicht zufriedenstellenden Rechercheergebnis, durchschnittlich zwei bis drei Recherchevorgänge unternehmen. Hier hat die letzte Anfrage ein Ergebnis geliefert. 
Abschließend werden noch einige Einzelbeispiele aus den Logfiles vorgestellt, die sich leicht erklären lassen, aber keinem bestimmten Muster folgen:

Bsp. 4.2.28

Erweiterte Suche: Verfasser

"Borggräfe, Henning Jansen, Christian"

Eingabe von zwei Verfassern in ein Feld erzielte keinen Treffer.

Bsp. 4.2.29

Erweiterte Suche: Titel

"AmoRLauf"

Die Suche nach diesem Titel, exakt wiedergegeben mit Großbuchstaben, erzielte den passenden Treffer.

Bsp. 4.2.30

Einfache Suche

"Budde, J. (2008). Bildungs(miss)erfolge von Jungen und Berufswahlverhalten bei Jungen/männlichen Jugendlichen, Bildungsforschung Band 23. BMBF: Bonn, Berlin."

Diese Eingabe in der einfachen Suche sieht sehr nach 'Copy and Paste' aus. Erfolgreich war diese Recherche nicht. 


\section{Bsp. 4.2.31}

Erweiterte Suche: Titel \& Verfasser

"Fangfragen im Vorgespräch souver" \& "Lüdemann, Carolin"

Erweiterte Suche: Titel \& Verfasser

"Fangfragen im vorstellungsgespräch souverän meistern" \& "Lüdemann, Carolin"

Die recherchierende Person hat entweder vergessen, den Titel zu vervollständigen, oder aber hat gehofft, wie bei Google, dennoch etwas zu finden. Da es aber im OPAC keine automatische Trunkierung gibt, war die erste Anfrage erfolglos. Die zweite hingegen erzielte einen Treffer.

Bsp. 4.2.32

Einfache Suche

"Die Weltwirtschaft Jg.2002, S.85-108"

Die exakte Eingabe dieses Aufsatzes erbrachte keinen Treffer, da, wie bereits erwähnt, keine einzelnen Aufsätze im OPAC erfasst werden.

\subsection{Rechercheverhalten im Katalog der UB Heidelberg}

Da die UB Heidelberg einen bereits erwähnten anderen Katalog für die Recherche bereithält, ist es interessant, auch deren Logfiles zu beobachten.

Den Schwerpunkt der Analyse soll hierbei der Vergleich zur Recherche im LBZ Rheinland-Pfalz bilden. Zentrale Fragestellungen waren: Gibt es Parallelen des Nutzerverhaltens bei der known-item search in beiden Katalogen und wenn ja, lassen sich die im vorhergehenden Kapitel herausgearbeiteten Verhaltensmuster bestätigen? 
Die Untersuchung der Heidelberger Logdateien spiegelt im Wesentlichen die Ergebnisse der Analyse der Daten des LBZ Rheinland-Pfalz.

Auffälligkeiten gibt es im Bezug einfache Suche versus erweiterte Suche. In HEIDI ist standardmäßig auf der Startseite ein Suchschlitz für die einfache Suche gesetzt. Die erweiterte Suche ist nur über einen weiteren Mausklick zu erreichen. Aus diesem Grund benutzen die meisten Nutzer die einfache Suche.

Diese Vorgehensweise hat eine größere Ergebnismenge zur Folge. Deshalb geben Nutzer hier tendenziell mehr Suchanfragen ein, um die Ergebnismenge zu verkleinern.

Erwähnenswert ist, dass einige Benutzer gerne Abkürzungen bei ihrer Recherche verwenden (Bsp. 4.3.1). Oft kann dies allerdings zu einem negativen Suchergebnis führen.

Bemerkenswert oft sind Rechercheanfragen mit einem kompletten und ziemlich langen Titel festzustellen. Ab und an ist auch der Zusatz, abgetrennt mit Doppelpunkt, Strichpunkt oder ähnliches in der Suche enthalten.

Boolesche Operatoren und Trunkierungen werden in HEIDI sehr sparsam, beziehungsweise überhaupt nicht genutzt. Kombinationen, mehrheitlich Autor und Titel oder Autor und Jahr, werden mittels dem Operator UND verknüpft.

Die Eingabe von Nachname Komma Vorname, also in normierter Form, ist bei HEIDI stärker ausgeprägt als zum Beispiel im OPAC der LBZ Rheinland-Pfalz.

Recherchen nach fremdsprachigen Medien sind in HEIDI sehr verbreitet und führen in der Regel zum gewünschten Ergebnis.

Was bei fremdsprachigen Titeln nicht auffällt, aber dafür bei deutschsprachigen Titeln um so mehr, ist die Groß- und Kleinschreibung. Für viele Fremdsprachen ist eine Kleinschreibung die Regel. Für deutschsprachige Titel macht sich der Nutzer häufiger die Mühe, auf Groß- und Kleinbuchstaben zu achten. Derselbe Aspekt tritt noch häufiger bei Personennamen auf. Hier besteht im Allgemeinen eine Tendenz zur Großschreibung.

Große Treffermengen, bedingt durch die bereits erwähnte einfache Suche, scheinen Nutzer stark abzuschrecken. Es werden meist sofort erneute Recherchen mithilfe der Hinzunahme von weiteren Wörtern oder durch die Verwendung von Kombinationen, angestellt. Null-Treffer veranlassen die Suchenden ebenfalls zu einer erneuten und veränderten Suchanfrage. Am Ende wird sehr oft das gewünschte Medium gefunden, auch wenn dafür mehrere Suchanfragen nötig waren. 
Beispiele aus der Praxis:

\section{Bsp. 4.3.1}

Erweiterte Suche: Titel

"sterben und tod"

Erweiterte Suche: Titel \& Autor

"sterben und tod" \& "Uni HD“

Erweiterte Suche: Titel \& Autor

"sterben und tod" \& "Universität"

Die recherchierende Person hat bei ihrer ersten Anfrage nach einem Titel zu viele Treffer erhalten, nämlich 187. Die Hinzunahme des Suchfeldes für einen Autor, in diesem Fall ein Herausgeber, in der zweiten Anfrage erbrachte keinen Erfolg, da dieser abgekürzt wurde. Erst die dritte Anfrage lieferte dann das gewünschte Ergebnis.

\section{Bsp. 4.3.2}

Erweiterte Suche: Titel

"Bellezza e lusso nell'Italia Antica. Immagini e documenti"

Erweiterte Suche: Titel

"Bellezza e lusso Italia Antica"

Erweiterte Suche: Titel

"Bellezza Italia Antica"

Die Recherche nach fremdsprachigen Titeln gestaltet sich, wie in diesem Fall, ab und an zu einem 'Geduldsspiel'. Teilweise wird, wie im vorliegenden Fall, der Titel nicht genau gewusst und falsch wiedergegeben. Beim dritten Anlauf wurde schließlich ein Medium gefunden. 


\section{Bsp. 4.3.3}

Erweiterte Suche: Freitext

"Bailey"

Erweiterte Suche: Freitext

"f. g. bailey"

Erweiterte Suche: Autor

"Bailey, Frederick G."

Auch in HEIDI haben die Nutzer das Bedürfnis, die Ergebnismenge so gering wie möglich zu halten. So wurde hier die Anzahl der Treffer von 2028 auf 92 und schließlich auf noch überschaubare 15 Anzeigen gesenkt.

\section{Bsp. 4.3.4}

Erweiterte Suche: Autor

"nowak"

Erweiterte Suche: Freitext \& Autor

"geschicht" \& "nowak"

Erweiterte Suche: Freitext \& Autor

"geschicht" \& "nowak, kurt"

Dieser Nutzer hat ebenfalls mehrere Anfragen benötigt, um auf das richtige Ergebnis zu stoßen. Zudem hat der Suchende bei Geschichte ein 'e' vergessen. Dies hat bei der Recherche aber keine gravierende Auswirkung. Hier verringerte sich die Trefferzahl von 392 über 80 auf neun Anzeigen. 


\title{
Bsp. 4.3.5
}

Einfache Suche

"Coaching durch systemisches Denken zu innovatviver Personalentwicklung"

Einfache Suche

"Coaching durch systemisches Denken zu innovativer Personalentwicklung"

Die Eingabe eines kompletten Titels, wie in diesem Fall, verleitet oft zu Rechtschreibfehlern. Vermutlich aufgrund der Tippfehlerkorrektur des Systems konnte der Nutzer noch zwei Ergebnisse erzielen.

\section{Bsp. 4.3.6}

\author{
Einfache Suche \\ "cicero orationes" \\ Einfache Suche \\ "cicero orationes albert clark"
}

Hier zeigt sich deutlich die Tendenz, mit geringem Aufwand, sprich der Eingabe von wenigen Wörtern, zu suchen. In diesem Fall bekam der Nutzer 273 Treffer. Der Entschluss zu einer erneuten und erweiterten Anfrage führte zu nur noch fünf Treffern.

\section{Bsp. 4.3.7}

Erweiterte Suche: Titel

"Wilhelm der Eroberer. Herzog der Normandie, König von England; 10281087"

Diese Eingabe war erfolgreich, ist aber wahrscheinlich vom Suchenden durch 'Copy and Paste' eingefügt worden. Diesen Sachverhalt kann man öfters finden. 


\subsection{Zusammenfassung}

Auf der Basis der Analyse von Logfiles aus zwei Bibliotheken lassen sich nun interessante und immer wiederkehrende Verhaltensmuster von Nutzern bei der knownitem search zusammenfassen.

Am häufigsten recherchieren die Nutzer mittels Titel beziehungsweise Titelstichwörtern; dies ist nach Betrachtung der Logfiles die meist genutzte Recherchevariante bei einer known-item search. Bei einer Suche nach einem Titel geben viele Nutzer häufig sehr wenig ein, was vor allem bei der einfachen Suche zu langen Trefferlisten führen kann (Bsp. 4.2.7). Doch tritt auch, gerade bei der Titelsuche, das Gegenteil auf. Nicht selten wird der komplette Titel, mit Stopp- und Füllwörtern oder sonstigen Satzzeichen, komplett eingegeben (Bsp. 4.2.30).

Schon etwas geringer sind die Recherchen nach einem Verfasser. Dieser wird häufig nur mittels Nachnamen gesucht, was bei der einfachen Suche zu großen Treffermengen führen kann. Wird er hingegen mit einem Vornamen gesucht, so ist eine normierte Eingabe, sprich Nachname Komma Vorname, seitens der Nutzer eher gering. Negative Auswirkungen hat dies bei den untersuchten OPACs allerdings keine (Bsp. 4.2.3).

Sowohl bei Titel- als auch Verfasserrecherchen wird überwiegend auf eine korrekte Groß- und Kleinschreibung geachtet, die für eine erfolgreiche Recherche nicht nötig wären (Bsp. 4.2.29).

Seltener werden Recherchen mittels Signatur oder ISBN vorgenommen. Oft führen diese Recherchen zu einer Nulltrefferanzeige, da sie nicht richtig oder unvollständig wiedergegeben wurden (Bsp. 4.2.13).

Zu einer Nulltrefferanzeige führen ebenfalls sehr häufig Rechtschreibfehler, gerade bei sehr langen Titeleingaben, falsch angewendete Suchoptionen oder Kombinationen und Abkürzungen (Bsp. 4.2.12).

Die allermeisten Nutzer geben nicht auf, sondern unternehmen weitere Recherchen. Diese werden dann oft mittels Kombinationen durchgeführt oder es wird nach einer Recherche mit der einfachen Suche, die meist sehr viele Ergebnisse liefert, in die erweiterte Suche gewechselt. Bei Kombinationen dominiert eindeutig die Suche mittels Verfasser und Titel. Verknüpft wird diese immer mit dem Booleschen Operator UND. Andere Operatoren treten so gut wie nie auf (Bsp. 4.2.19). Äußerst selten ist 
die Verwendung von Trunkierungen. Werden sie jedoch eingesetzt, so führen sie in der Regel auch zum Erfolg (Bsp. 4.2.15). 


\section{Nutzerwissen und Verbesserungsmöglichkeiten}

Nachdem herausgearbeitet wurde, wie Nutzer bei einer known-item search vorgehen, soll nun Ursachenforschung betrieben werden. Warum bestimmte Nutzer so und nicht anders recherchiert haben, lässt sich natürlich im Nachhinein nicht mehr sicher feststellen. Es lassen sich jedoch aus den im vorhergehenden Kapitel besonders oft vorkommenden Verhaltensmustern Rückschlüsse ziehen. Aus diesen Überlegungen werden anschließend auf der Basis des analysierten Rechercheverhaltens Verbesserungsmöglichkeiten aufgezeigt, die vor allem die known-item search betreffen.

\subsection{Recherchevorgänge und ihre Intentionen}

Die Eingabe von Nachnamen ohne den dazugehörigen Vornamen kommt sehr häufig vor. Dies führt in vielen Fällen zu sehr großen Treffermengen, vor allem dann, wenn der Nachname in der einfachen Suche eingegeben wurde. Systembedingt wird über alle Felder alles gesucht, also alle Treffer, die diesen Namen enthalten, möglicherweise auch Sachtitel, werden angezeigt (Bsp. 4.2.3).

Nutzer scheinen im Allgemeinen nicht daran zu denken, dass sich hinter einem Familiennamen sehr viele Medien verbergen können. Es wird höchstwahrscheinlich damit gerechnet, dass die gewünschte Person sofort mit dem richtigen Titel erscheint. Dass es aber noch Personen mit dem gleichen Nachnamen gibt, oder dass eine Person an sehr vielen Werken beteiligt war, ist manchen Nutzern nicht sofort bewusst.

Dies hat zur Folge, dass erneute Rechercheanfragen gestartet werden. Dieses Mal jedoch mit Vornamen, der den meisten Nutzern anscheinend bekannt ist und zu einem oftmals befriedigenden Ergebnis führt (Bsp. 4.2.1).

In einigen Fällen wird der Vorname jedoch abgekürzt auf den Anfangsbuchstaben wiedergegeben oder es werden Funktionsbezeichnungen, ebenfalls abgekürzt, hinzugefügt. Da es hierfür keine Indexierung gibt, werden abgekürzte Wörter bei einer Recherche nicht gefunden (Bsp. 4.2.20).

So liegt hier wohl eine Mischung aus Unwissenheit über die Menge an Datensätze im Katalog, wie auch eine gewisse Trägheit bei der Eingabe der Nutzer vor. 
Ähnliches ist bei der Eingabe von Sachtiteln zu beobachten. Oftmals werden hier nur einzelne, nicht aussagekräftige Wörter verwendet. Manchmal beschränkt sich der Suchende auch nur auf ein einziges Wort. In beiden Fällen ist eine größere Ergebnismenge nicht sehr überraschend (Bsp. 4.2.9).

Um doch noch das gewünschte Medium zu finden, wird entweder der Sachtitel um weitere Wörter ergänzt oder aber die Titelwörter in das Titelfeld eingetragen. Jedoch tritt bei der Recherche nach Titeln auch das umgekehrte Phänomen auf, nämlich die Eingabe von zu vielen Wörtern. Dies kann dazu führen, dass ein sehr langer Sachtitel, teilweise mit Zusatz, komplett eingegeben wird (Bsp. 4.2.6). Die Chancen, damit auf den richtigen Treffer zu stoßen, sind ambivalent. Entweder hat der Nutzer vermutlich durch 'Copy and Paste' den Titel aus einer anderen Quelle kopiert und stößt möglicherweise sofort auf den gewünschten Treffer, vertippt sich bei der Eingabe und es folgt ein negatives Rechercheergebnis oder das gesuchte Medium ist nicht im Bestand.

Die Annahme, dass 'Copy and Paste' verwendet wurde, rührt daher, dass einige Titel mit Bindestrichen, Punkten oder Doppelpunkten und Semikolon eingegeben wurden. Dies ist häufiger im Internet beziehungsweise auf dortigen Verkaufsplattformen wie 'Amazon' zu finden.

Bandangaben oder ähnliches werden von manchen Nutzern bei der Suche zum Titel hinzugefügt beziehungsweise beim 'Copy and Paste' mit übernommen. Auf Grund der Nicht-Indexierung dieser Angaben wird die Rechercheanfrage aber negativ beantwortet (Bsp. 4.2.27 / Bsp. 4.2.32).

Öfters werden auch beispielsweise Präpositionen verwechselt oder der Titel in anderer Weise nicht richtig wiedergegeben (Bsp. 4.2.19), was wiederum zu Null-Treffern führt oder ein anderes Medium als gewünscht anzeigt.

Viele Nutzer finden die Balance zwischen zu-wenig-Eingegeben und zu-viel- Eingegeben offenbar nicht und machen sich dadurch mehr Arbeit als nötig.

Die Recherche nach Personen als auch nach Titeln lässt sich auffälligerweise durch das Eingeben von nur sehr wenigen Daten zusammenfassen. Entweder wird, wie schon erwähnt, nur der Nachname eingegeben oder zu wenige Titelwörter.

Dies sind die häufigsten Gründe für Trefferlisten, die über zehn Ergebnisse hinausgehen.

Einige Nutzer, so kann man schließen, wollen mit möglichst geringem Aufwand schnellstmöglich an Ergebnisse kommen. Dies ist wohl eine Verhaltensweise, die auf 
Internetsuchmaschinen wie zum Beispiel Google zurückzuführen ist. Bei Google allerdings findet ein Ranking statt, das dazu führt, dass häufig gesuchte Seiten weit vorne erscheinen. Hier reicht also oftmals die Eingabe von wenigen Begriffen.

Bei OPACs findet hingegen meist kein Ranking statt. So kann es sein, dass ein häufig recherchiertes Buch immer gleichweit hinten in der Trefferliste erscheint, wenn bei der Recherche keine ausreichende Einschränkung vorgenommen wurde.

Konträr zu dieser Googlementalität verhält sich die Beachtung von korrekter Großund Kleinschreibung, obwohl sie für eine erfolgreiche Recherche keine Rolle spielen sollte (Bsp. 4.2.29).

Wiederkehrend und auch ein Grund für ein negatives Rechercheergebnis sind die Eingaben in falsche Kategorien. Es wird häufiger ein falsches Feld für die Recherche verwendet (Bsp. 4.2.11). So ist zum Beispiel der Autor im Feld 'Titel' zu finden oder aber umgekehrt.

Die meisten Nutzer wissen vermutlich nicht, dass der OPAC ein mächtiges Rechercheinstrument ist, wenn man inn richtig einsetzt. So werden zum Beispiel im LBZ Rheinland-Pfalz bei der erweiterten Suche oft nur die drei Suchoptionen verwendet, die schon voreingestellt sind. Das Drop-Down Menü, das etwa die Einstellung 'Ganzer Titel' zulässt, wird so gut wie nie benutzt.

Auch Kombinationen mittels Booleschem Operator UND scheinen für manche Nutzer ein Hindernis zu sein. Hier ist bei der Einfachen Suche zu erkennen, dass Titel, Verlag oder Autor mit Satzeichen getrennt werden oder sogar irgendwelche Sonderzeichen für die Trennung der Elemente zum Einsatz kommen (Bsp. 4.2.4). Dies muss zwar kein negatives Rechercheergebnis hervorrufen, da eine 'ordentliche' Indexierung dies auffangen kann. Es deutet aber daraufhin, dass mancher Nutzer im Hinblick auf Kombinationen nicht ganz sicher ist. Es wird zwar erkannt, dass zum Beispiel Verfasser und Verlag zwei verschiedene Elemente sind, aber wie diese bei der einfachen Suche einzugeben sind, irritiert so manchen (Bsp. 4.2.30).

Fehler bei Kombinationen finden auch bei der erweiterten Suche statt. So wird hier zum Beispiel Verlag und Verlagsort im Feld 'Verlag' untergebracht, anstatt eine weitere Kategorie für den Verlagsort zu wählen (Bsp. 4.2.11).

Ob vielen Benutzern die Booleschen Operatoren bekannt sind, ist sehr fraglich. In der Regel wir nur mit dem Operator UND, der schon voreingestellt ist, recherchiert. So lässt sich vermuten, dass viele Nutzer nichts oder nur sehr wenig über die Existenz und Möglichkeiten von Booleschen Operatoren wissen. 
Die known-item search mittels ISBN oder Signatur geschieht recht selten. Es ist wohl anzunehmen, dass entweder das Bibliothekspersonal mit diesen Modi recherchiert oder Nutzer die ISBN zum Beispiel aus dem Internet kopieren. Signaturen geben wohl noch weniger Nutzer ein als die ISBN. Es muss die Signatur schon im Vorfeld bekannt sein. Eventuell kennen Nutzer die Signatur von vorherigen Ausleihen.

Der manchmal komplizierte Aufbau von Signaturen bereitet bei der Eingabe teilweise Probleme, die öfters in einer Nulltrefferanzeige enden (Bsp. 4.2.14).

Trunkierungen können gerade bei Signaturen oder ISBNs, aber auch bei Verfasserbzw. Titelrecherchen eine bequeme Lösung der Suche darstellen. Leider wird diese Möglichkeit nur selten benutzt. Die Mehrzahl der Nutzer, so hat es jedenfalls den Anschein, nutzt und kennt diese Hilfe bei der Recherche nicht. Eingesetzte Trunkierungen, so zeigen es die Logfiles, führen mehrheitlich zu einer positiven Antwort (Bsp. 4.2.15).

Die bereits erwähnten Fälle für Nulltrefferanzeigen kommen zwar nicht selten vor, sind aber nicht die einzigen Gründe für negativ beantwortete Suchanfragen.

In erster Linie dafür verantwortlich sind hier Rechtschreibfehler und inkorrekte Angaben (Bsp. 4.2.10). Kompliziertere Personennamen werden zum Beispiel nicht immer korrekt eingegeben und bei Titeln sind die Gründe noch vielschichtiger. So werden etwa Pluralformen gebildet, wo eigentlich eine Singularform stehen sollte, oder Adjektive werden in substantivierter Form recherchiert.

Zu beachten ist aber, dass in einigen Fällen die gewünschten Medien nicht im Bestand der Bibliothek zu finden sind. Deshalb sind nicht alle Nulltrefferanzeigen unbedingt aus einem negativen Blickwinkel zu betrachten.

Was ebenfalls negative Auswirkungen auf die Recherche haben kann, ist die Materialart beziehungsweise Medienform. Es geschieht immer wieder, dass Nutzer zum Beispiel einen Titel, manchmal sogar mit Autor, eingeben und nichts finden. Die Lösung ist relativ simpel. Es handelt sich nämlich um einen Aufsatzartikel aus einer Zeitschrift, der nicht im OPAC verzeichnet ist. Dieses Faktum stellt für viele Nutzer ein großes Hindernis dar, das nicht immer überwunden wird. (Bsp. 4.2.25) 
Nutzer wissen also durchaus, was sie wollen, wissen aber zugleich nicht, wie die Bibliothek diese Dinge erschließt beziehungsweise nicht erschließt und ob das gewünschte Schrifttum recherchierbar ist oder nicht.

Positiv ist aber, dass die known-item search im Großen und Ganzen den Nutzern meist schnell ein zufriedenstellendes Ergebnis liefert. So findet ein Großteil der Recherchierenden oft schon nach der ersten Suchanfrage das gewünschte Medium. Auch die Trefferanzahl, die überwiegend unter zehn Treffern liegt, deutet auf eine für den Suchenden zufriedenstellende Recherche hin. Jedoch muss auch darauf hingewiesen werden, dass ein Treffer nicht immer positiv sein muss, da zum Beispiel auch etwas angezeigt werden kann, dass der Nutzer nicht möchte, sozusagen das falsche Medium.

Bei einer Unübersichtlichkeit, die bei vielen schon bei mehr als zehn Treffern beginnt oder einem negativen Rechercheergebnis wird so gut wie immer eine zweite beziehungsweise dritte, größtenteils positiv verlaufende, Suche begonnen.

\subsection{Verbesserungsmöglichkeiten der Recherche}

Den perfekten OPAC wird es wohl, wie eingangs erwähnt, nie geben. Es können aber immer wieder Verbesserungen eingeführt werden, um auf technische Innovationen oder geänderte Benutzerbedürfnisse zu reagieren. Auch ein Blick 'über den Tellerrand hinaus' in Richtung Internetsuchmaschinen kann manchmal nicht schaden. Denn gerade hier entwickeln sich neue Techniken für Suchmaschinen, wovon auch der OPAC profitieren kann, sehr schnell. Im Folgenden sollen daher einige Ideen zur Verbesserung aufgezählt werden.

Technische Verbesserungen bzw. Innovationen am OPAC müssen stärker gefördert und ausgebaut werden. Nur so lassen sich effektive und bedienungs- und recherchefreundliche Kataloge gestalten. Technisch müssen Bibliotheken dabei keine neuen Wege gehen und das 'Rad neu erfinden'. Viele innovative Möglichkeiten in puncto Recherche lassen sich bei Google oder ähnlichen Internetsuchmaschinen finden. Hier wäre an erster Stelle ein Programm zur automatischen Rechtschreibkorrektur zu 
nennen. ${ }^{44}$ Solche Programme kommen bei Recherchen im Internet beziehungsweise auf Internetseiten schon länger erfolgreich zum Einsatz.

Wichtig für Bibliothekskataloge sind hier insbesondere die Verbesserungen von orthografischen Fehlern.

Bei orthografischen Fehlern wird geprüft, welche Wörter mit kürzester LevenshteinDistanz (Bestimmung von Ähnlichkeiten bei Zeichenketten) vorkommen und diese werden in absteigender Häufigkeit geliefert. Die Anfrage wird dabei geparst (zerlegt). Zu jedem Term der Anfrage werden Alternativterme ausgeben, dann werden alle Kombinationen durchprobiert (ersetzen eines einzelnen Terms in der boole'schen Anfrage) und verglichen, welche Kombination die meisten Treffer liefert. Hinzu kommen noch Prüfungen nach häufigen Fehlern, wie zum Beispiel abgekürzte Vornamen. ${ }^{45}$ Eingesetzt wird ein solches Programm schon seit längerem in HEIDI (Bsp. 4.3.5). ${ }^{46}$

Die automatische Wortformreduktion, auch bekannt unter Stemming, kann bei einer Recherche nach dem Sachtitel eine weitere Methode gegen Rechtschreibfehler sein. Bei diesem Verfahren werden verschiedene Formen eines Wortes auf dessen lexikalische Grundform zurückgeführt. Durch Entfernung von Flexionsendungen und Derivationssuffixe bleibt ein Wortkern übrig, zum Beispiel freizügig wird zu freizüg. ${ }^{47} \mathrm{Es}$ können somit Singular- oder Pluralformen und Flexionsformen gefunden werden. Zum Beispiel bekommt man statt freizügig in diesem Fall auch freizügige geliefert. Bei einer Recherche nach dem Sachtitel kann diese Methode vorbeugend gegen Rechtschreibfehler und ungenaue Eingaben helfen und zu besseren Suchergebnissen führen. Ein gutes Stemming-Programm bietet zum Beispiel Summon von Serials Solutions. ${ }^{48}$

Für welche syntaktische Nachbearbeitungsprogramme sich Bibliotheken auch entscheiden, wichtig ist die sinnvolle Einbringung dieser Programme in den OPAC. Die vom Programm erkannten Fehler können entweder stillschweigend im Hintergrund behoben werden, oder aber es erscheint eine Anzeige die lauten könnte 'Meinten Sie

\footnotetext{
${ }^{44}$ Bsp. Kneifel, Fabienne (2009): Mit Web 2.0 zum Online-Katalog der nächsten Generation, S. 54

${ }^{45}$ Vgl. Maylein, L. (2012): E-Mail vom 02.02.2012

${ }^{46}$ Bsp. UB Heidelberg (o.J.): HEIDI. URL: http://www.ub.uni-heidelberg.de/helios/kataloge/heidi.html

${ }^{47}$ Vgl. Lang, J. und Kowatschew, R. (2002): HS Information Retrieval: Stemming, S. 4 (elektronische Quelle)

${ }^{48}$ Serials Solutions (o.J.): The Summon ${ }^{\mathrm{TM}}$ Service.

URL: http://www.serialssolutions.com/discovery/summon/
} 
...' oder ähnliches. Auch eine Auflistung von verwandten Begriffen oder ähnlichen, im Bestand vorhandenen Sachtiteln könnten bei einer Nulltrefferanzeige erscheinen.

Ähnlich wie die oben genannten Programme funktioniert eine Recherche mittels der Einstellung unscharfe Suche oder auch Fuzzy-Suche. Ein eingegebener Suchbegriff hat bei dieser Methode den Vorteil, auch ohne hinterlegte Wortlisten ähnliche Wörter aus dem Datenbestand des OPACs zu ermitteln. Werden noch Synonymlisten hinterlegt, können zusätzlich noch weitere verwandte Begriffe zum eingegebenen Suchwort gefunden werden. Für den Nutzer hat dies den Vorteil, dass er schon im Vorfeld die unscharfe Suche einstellen kann und weiß, die eingegebene Rechercheanfrage muss nicht zu 100\% mit dem exakten Namen des Verfassers übereinstimmen oder der exakte Sachtitel sein. Diese Recherchemethode bietet also einen Weg, auch mit inkorrekten Angaben schnell und bequem zu einem zufriedenstellenden Ergebnis zu gelangen. Die unscharfe Suche wird teilweise im Bibliothekssektor schon eingesetzt, so zum Beispiel in der Staatsbibliothek zu Berlin. ${ }^{49}$ Wird hier z.B. eine Person als Levensstein eingegeben, so werden auch Ergebnisse zu Levenstejn, Levinshtein oder Levinstein gefunden. Dies sind allerdings keine Formen aus dem Normdatensatz.

Das Abfangen von Bedienungsfehlern im Bereich von Suchoptionen ist eine Möglichkeit, Nutzern bei der Recherche zu helfen, entweder ein passendes Ergebnis zu bekommen, wenn Null-Treffer angezeigt werden, oder bei einer zu großen Treffermenge die Anzahl der Treffer durch die Wahl einer passenden Suchoption zu minimieren. Für die Praxis könnte dies so aussehen, dass zum Beispiel bei einer zu groBen Treffermenge eine Anzeige erscheint, die den Nutzer hinweist, mit der Suchoption ' $X Y$ ' seine Ergebnisse weiter einschränken zu können. Im umgekehrten Fall, nämlich Null-Treffern bei einer bestimmten Suchoption, könnte das System den Suchbegriff aus der erweiterten Suche automatisch in die einfache Suche zu einer erneuten Recherche übernehmen.

Die Möglichkeit zum Browsen besteht mittlerweile in vielen OPACs, so auch in HEIDI oder im LBZ Rheinland-Pfalz. Hier besteht im Index zu den einzelnen Suchfeldern

\footnotetext{
${ }^{49}$ Staatsbibliothek zu Berlin (o.J.): StaBiKat. Online-Katalog der Staatsbibliothek zu Berlin. URL: http://stabikat.de//
} 
die Möglichkeit diese Indizes mittels browsing zu 'durchstöbern'. Es müsste allerdings auf diese Möglichkeit der Recherche besser hingewiesen werden. Im LBZ Rheinland-Pfalz könnte dies zum Beispiel in der Box mit den 'Tipps zur Suche' erwähnt werden. Jedoch ist anzumerken, dass sowohl im LBZ Rheinland-Pfalz, wie auch in HEIDI, die Recherche mittels Index ausführlicher im Hilfetext erklärt wird. Sehr hilfreich wäre auch ein Index wie in HEIDI, der bei einer Eingabe automatisch erscheint. Diese Autovervollständigung mittels Drop-down Liste würde eine weitere Anpassung an die Suchmaschinentechnologie bedeuten und könnte somit viele Nutzer bei der known-item search schnellerer und sicherer zum Ziel führen. ${ }^{50}$

Sehr problematisch sind für viele Nutzer Aufsatzartikel und ähnliches, die oft nicht selbstständig erschlossen und aufgeführt werden.

Hier hat zum Beispiel die UB Freiburg eine vernünftige Lösung dieses Problems gefunden. Sie kombiniert die Recherche aus ihrem eignen Bestand mit der Recherche in einem RDS-Index (Resource Discovery System). Somit können die Nutzer hier beispielsweise nicht nur Bücher, sondern auch Artikel von elektronischen Ressourcen und Nationallizenzen recherchieren und abrufen. Diese Kombination kann auf zwei verschiedene Arten stattfinden:

1. „Die lokalen Katalogdaten werden beim Anbieter des RDS-Index gemeinsam mit dem RDS-Index geladen und in einer einheitlichen Suchmaske angeboten. Drilldowns (Facetten) ermöglichen nicht nur die Einschränkung auf spezifische Sachfragen, sondern auch die Einschränkung auf den lokalen Bestand. So entsteht dann wieder eine ausschließliche Sicht auf die lokalen Daten.

2. Die lokalen Daten werden in einem eigenen, lokal aufgebauten Suchmaschinenindex geladen und parallel mit einer Suche im RDS-Index angeboten. Die Daten werden dabei nicht gemischt. Lokale Sicht und RDS-Sicht werden in getrennten Fenstern dargestellt.“51

Nachteile beim RDS-Index bestehen unter anderem noch bei fachwissenschaftlichen Recherchen mittels Thesaurus, da dies noch nicht möglich ist. Ein ähnliches System bietet zum Beispiel auch die UB Konstanz ihren Nutzern an.

\footnotetext{
${ }^{50} \mathrm{Vgl.}$ Wiesenmüller, Heidrun (2012): Informationskompetenz und Bibliothekskataloge (im Druck)

${ }^{51}$ Ullrich, H. und Ruppert, A. (2011): Katalog plus - die Freiburger Lösung zur Kombination von lokalem Katalog und globalem RDS-Index, S. 11
} 
Weitere Möglichkeiten, die zu einer Erleichterung der Recherche beitragen können, sind zum Beispiel eine übersichtliche und ansprechende Darstellung der Suchoberfläche und ausreichend Hilfetexte oder Tipps zur Recherche. Diese dürfen aber nicht kleingedruckt oder versteckt sein, sondern müssen eine leicht zu findende 'Anlaufstelle' für ratlose Suchende bieten. Dabei sollte die Seite nicht überladen sein und von den Farben nicht zu 'knallig' wirken. Einfache Navigation und selbsterklärende Icons oder Felder sind ein wichtiger Schritt für den Beginn einer erfolgreichen Recherche. Hierzu hat Alexander Weimar einige interessante Aspekte herausgearbeitet. ${ }^{52}$ Unabdingbar sind auch Ratschläge zu einer erneuten Recherche auf Grund eines negativen Suchergebnisses.

Desweiteren sollten Hilfen in OPACs barrierefrei gestaltet sind, um auch behinderten Menschen einen leichten Zugang zur Recherche zu ermöglichen. ${ }^{53}$

Oftmals können schon kleinere Verbesserungen zu einer erfolgreichen Recherche beitragen. Hierzu zählt zum Beispiel eine automatische Trunkierung durch das System. Bei dieser Methode könnten so viele Zeichen beziehungsweise Buchstaben des Sucheintrags entfernt werden, bis etwas stehen bleibt, das mit einem Eintrag im Katalog übereinstimmt. Mit einer Anzeige 'Meinten Sie ...?' könnte dieser Vorschlag dem Nutzer präsentiert werden. Null-Treffer wie in Beispiel 4.2.6 könnten dadurch vermieden werden.

Das Thema Indexierung bietet noch eine weitere interessante Möglichkeit, die die Recherche verbessern könnte. Da wie in Beispiel 4.2.11 einige Nutzer zwei Verfasser in ein Feld eintragen, was prinzipiell aus Nutzersicht nicht falsch ist, aber systembedingt zu Null-Treffern führt, besteht hier Handlungsbedarf. So könnte man durch eine Stichwortindexierung dieses Problem lösen und den Nutzern eine einfachere und schnellere Recherche ermöglichen. Auch das Problem der Jahreszahlen bei Zeitschriften kann bei einer sinnvollen Indexierung umgangen werden.

Beispiel 4.2.11 liefert den Anlass für eine weitere und ebenfalls sinnvolle Verbesserung der Suchkategorien. So wäre es eine Überlegung wert, weniger häufig genutzte Felder wie Verlag und Verlagsort zusammenzulegen. Durch eine entsprechende Indexierung müsste sich der Nutzer keine Gedanken mehr über den richtigen Feldein-

\footnotetext{
${ }^{52}$ Vgl. Weimar, A. (2004): Inhaltserschließung und OPAC, S. $47 \mathrm{ff}$.

${ }^{53}$ Vgl.: Greifeneder, Elke (2007): Online-Hilfen in OPACs: Analyse deutscher UniversitätsOnlinekataloge, S. $20 \mathrm{f}$.
} 
trag machen und die Anzahl der Suchfelder wird zudem reduziert und somit übersichtlicher.

Eine weitere, aber nicht idealtypische Lösung, sind Nutzerschulungen. Diese bereits seit langem existierende Methode könnte, wenn keine andere Lösung in Sicht ist, ausgebaut werden. Am besten wäre dabei natürlich, wenn Nutzerschulungen in den Studienalltag eingebaut werden würden. Für alle Erstsemester könnte es so zum Beispiel verpflichtend eine Vorlesung zum Thema 'Medienrecherche' oder ähnliches geben.

Interessant ist beim Thema Nutzerschulung auch das Feld der Tutorials, die auf virtuelle Weise den Nutzer an den OPAC heranführen. Hier gibt es schon interessante Möglichkeiten, wie zum Beispiel das Tutorial zur Online-Recherche der UB Bielefeld, das gezielt auf den Recherchebedarf der Nutzer eingeht. ${ }^{54}$

\subsection{Positive und negative Rechercheaspekte des LBZ Rheinland-Pfalz OPACs}

In diesem Kapitel werden ausgewählte positive, aber auch negative Eigenschaften und Funktionen des LBZ Rheinland-Pfalz OPACs aufgezeigt. Die bereits im vorangegangenen Kapitel erwähnten Vorschläge sollen hierbei nicht nochmals aufgegriffen werden.

Unter den positiven Aspekten des OPACs des LBZ Rheinland-Pfalz ist zunächst die Übersichtlichkeit der Suchmaske zu nennen. Diese ist nicht überladen oder verwirrend, sondern bietet eine optisch gute Ausganssituation für die Recherche. Außerdem ist der OPAC komplett in einer englischen Version verfügbar, was die Recherche für Nutzer mit wenig beziehungsweise keinen Deutschkenntnissen erleichtert. Tipps zur Suche sind direkt auf der Rechercheseite zu finden. Sie bieten in kurzer und knapper Form verständliche Hinweise zur Suche. Dem Recherchierenden stehen 15 Suchoptionen, fünf Möglichkeiten zur Einschränkung der Recherche und vier Sucheinstellungen zur individuellen Anpassung der Recherche an die eigenen Bedürfnisse, zur Verfügung. Es ist jedoch fraglich, ob dieses Instrumentarium wirklich genutzt und überhaupt vom Nutzer verstanden wird. Die untersuchten Logfiles haben

\footnotetext{
${ }^{54}$ UB Bielefeld und Senst, Erik (2010): Tutorial zur Online-Recherche. URL: http://www.ub.uni- bielefeld.de/help/tutorial/
} 
gezeigt, dass nur etwa ein Drittel der 15 vorhanden Optionen zur Recherche genutzt werden.

Das vorhandene Drop-down Menü wird, nach Betrachtung der Logfiles, von einer Mehrheit der Nutzer nicht angenommen.Daher gilt es zu überlegen, ob eine Reduktion der Suchfelder sinnvoll oder aber eine veränderte Darstellung für die Nutzer besser wäre.

Ein übersichtlicher und detaillierter Hilfetext bietet dem Suchenden eine ausführliche und gut aufbereitete Anlaufstelle bei Fragen und Problemen zum OPAC und zur Recherche.

Hat der Nutzer mit seiner Anfrage etwas gefunden, so steht inm die Möglichkeit zur Sortierung der Trefferliste zur Verfügung. Ebenfalls sind Tipps zur Trefferliste aufgelistet. Mit der Suchhistorie sind vorhergehende Suchanfragen einsehbar und zur weiteren Recherche verwendbar.

Bei einer negativen Recherche erscheinen gute und hilfreiche Tipps zu einer Weiterrecherche.

Aber es lassen sich auch, auf diesen OPAC beziehend, negative Punkte finden.

So ist die bereits erwähnte Hilfe etwas versteckt in der Auswahlleiste angebracht und nicht gesondert hervorgehoben. Es gibt zudem auch keine direkte Hilfe hinter den Suchschlitzen beziehungsweise zu den einzelnen Suchoptionen. Bei den ansonsten guten Tipps bei Nulltrefferanzeigen ist kein Hinweis zu finden, der auf die Auskunft als Anlaufstelle verweisen würde.

Das große Plus des LBZ Rheinland-Pfalz OPACs liegt sicherlich im Vorhandensein nur einer Suchmaske. Diese beinhaltet sowohl eine einfache Suche, hier freie Suche genannt und darunterstehend, die erweiterte Suche.

Eine separate einfache Suche ist auf der Homepage 'gut versteckt' und wird daher, nach Auswertung der Logfiles, nicht so oft benutzt wie bei anderen OPACs. Bietet man den Nutzern als Einstieg sowohl eine einfache Suche, als auch eine erweiterte Suche an, so werden möglicherweise bedingt durch die bereits erwähnte Nutzerklientel des LBZ, meist Suchfelder der erweiterten Suche benutzt. Dies hat in vielen Fällen weniger Recherchevorgänge zur Folge, da die Ergebnismenge von vorneherein durch Auswahl der passenden Suchoption übersichtlich ausfällt. So kommt der Nutzer oft schnell zum gewünschten Ergebnis. Natürlich kann auch der gegenteilige Effekt auftreten. Nicht allen Nutzern sind die Suchoptionen vertraut und dies führt gelegentlich zu Falscheingaben, die Null-Treffer zur Folge haben (Bsp. 4.2.11). 


\subsection{Spezifika des Heidelberger OPACs}

HEIDI hat viele Ähnlichkeiten mit dem OPAC des LBZ Rheinland-Pfalz. Diese sollen hier nicht noch einmal aufgeführt werden.

HEIDI bietet, im Gegensatz zu manch anderen OPACs, eine Einstellung zur Veränderung der Schriftgröße. Dies ist ein Schritt in Richtung barrierefreier OPAC und kommt so körperlich beeinträchtigten Nutzern stärker entgegen. Der sehr ausführliche Hilfetext ist mit anschaulichen Screenshots sehr verständlich gestaltet. Der Nutzer hat auch die Möglichkeit, mittels Links zu anderen Quellen zu gelangen. Bei Eingaben in Felder, die mit einem Index hinterlegt sind, werden dem Recherchierenden während der Eingabe in ein Suchfeld, wie zum Beispiel Titel, bereits Vorschläge durch einen automatisch erscheinenden Index zur Vervollständigung unterbreitet. Bei der Suche nach dem Autor funktioniert dies allerdings nur, wenn dieser normiert, also Nachname, Vorname eingegeben wird.

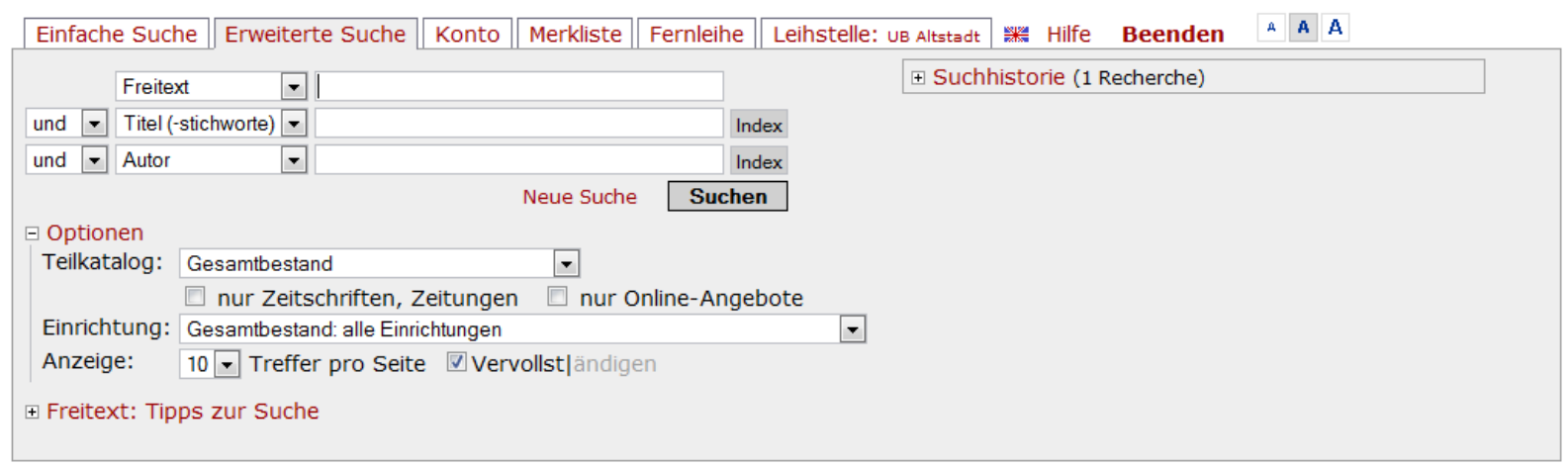

Abb. 11: HEIDI. Rechts unten die Möglichkeit 'Vervollständigen'.

Die erweiterte Suche bietet 'nur' drei Suchschlitze, wovon einer standardmäßig als Freitextsuche voreingestellt ist. Bei 22 Suchoptionen, die die UB Heidelberg bietet, sind dies etwas wenig voreingestellte Suchschlitze. Es ist, nach Betrachtung der Logfiles, davon auszugehen, dass der Nutzer meist nur die Suchoptionen in seine Recherche einbezieht, die bereits vorgegeben sind. 


\subsection{Untersuchungsergebnisse im Kontext der Literatur}

Es ist schwierig, die aus dieser Arbeit gewonnen Ergebnisse mit denen aus der gängigen Literatur zu Logfileuntersuchungen in Bibliotheken zu vergleichen, da nach meinem Wissen noch keine Studie dieser Art der tiefgehenden qualitativen Logfileanalyse existiert.

Jedoch lassen sich einige Parallelen aus der Literatur zu den hier gewonnen Ergebnissen herstellen. So lässt sich in dem Artikel von Malliari und Kyriaki-Manessi Angaben zur Verteilung der known-item search auf einzelne Suchoptionen finden. Demnach suchen $41 \%$ der Nutzer in dieser Studie mittels Titel, 27\% mit dem Autor und $8 \%$ via ISBN beziehungsweise Signatur. ${ }^{55}$ Der Beitrag erwähnt zudem, dass Nutzer die known-item search einer thematischen Suche vorziehen. Gründe hierfür sind aus Sicht der Nutzer eine einfachere und weniger fehleranfällige Suche als mit unbekanntem Schlagwortvokabular. ${ }^{56}$ Vermutlich hat sich auch der Einstieg für eine Recherche in Zeiten des Internets verändert. Viele Nutzer recherchieren bereits im Vorfeld in Internetsuchmaschinen, Wikipedia, Amazon oder ähnlichen Plattformen. Vermuten lässt sich dies durch die oftmals exakte Eingabe mittels 'Copy and Paste' in Bibliothekskatalogen.

Die Literatur verweist auf ein eindeutiges Ranking in Bezug auf die Nutzung von Suchoptionen. Platz eins belegt die Suche mittels Titel beziehungsweise Titelstichwörter, die je nach Artikel, zwischen $41 \%$ bis $61 \%$ liegt. Auf Platz zwei folgt eine Recherche nach Autor oder Verfasser mit $27 \%$ bis $46 \%$ aller Rechercheanfragen. An dritter Stelle folgt laut Literatur meist die thematische Suche via Schlagwort. Diese liegt oft zwischen $20 \%$ bis $30 \%$ in der gängigen Literatur. Andere Suchaspekte wie ISBN oder Signatur sind marginal und wurden entweder nicht mitgezählt oder liegen im einstelligen Prozentbereich.

Bei Kombinationen von Suchoptionen dominiert, und wird oft als einzige Verknüpfung explizit erwähnt, die Recherche mittels Titel und Autor. Allerdings kommen Kombinationen nicht sehr häufig vor, so jedenfalls das Ergebnis von Malliari und KyriakiManessi. ${ }^{57}$

\footnotetext{
${ }^{55}$ Vgl. Malliari, A. und Kyriaki-Manessi, D. (2007): Users' behaviour patterns in academic libraries' OPACs, S. 115

${ }^{56}$ Vgl. ebd., S. 117

${ }^{57}$ Vgl. ebd., S. 118
} 


\section{Erfahrungen aus der Praxis}

Die Logfile-Analysen bestehen, wie bereits erwähnt, nur aus Zahlen und statistischen Angaben. Es ist nicht mehr mit völliger Sicherheit die Absicht des Nutzers bei einer Recherche im Nachhinein festzustellen. Auch die ausgewerteten Daten in dieser Arbeit sind nur eine Momentaufnahme, ein minimaler Ausschnitt, von unzähligen Rechercheanfragen, die tagtäglich an deutschen wissenschaftlichen Bibliotheken getätigt werden.

Die Literatur mag hier Vergleichswerte liefern, diese sind aber ebenfalls nur Momentaufnahmen, die teilweise schon etwas älter sind. Zudem stammen viele davon aus dem Ausland und dort sind Bibliotheksnutzer möglicherweise anders geschult beziehungsweise haben ein anderes Rechercheverhalten als hierzulande. Die OPACs dort sind darüber hinaus teilweise anders aufgebaut als bei uns.

So ist es sinnvoll, die in dieser Arbeit gewonnen Zahlen, Statistiken und Feststellungen mit Beispielen und Erkenntnissen aus der Praxis des Auskunftsdienstes zu unterlegen.

\subsection{Befragungen mittels Fragebögen}

Als Methode für eine Erhebung von Daten aus der Praxis diente eine Umfrage mittels Fragebogen. Mit dieser Methode konnten über E-Mail schnell mehrere Fragebögen verschickt werden. Der Fragebogen selbst war mit zwei Word-Seiten und insgesamt acht Fragen relativ schnell zu beantworten (siehe Anhang C).

Verschickt wurde der Fragebogen an acht größere wissenschaftliche Bibliotheken in Baden-Württemberg und Rheinland-Pfalz. Empfänger war in der Regel jeweils der Leiter beziehungsweise die Leiterin der Informationsabteilung oder einer ähnlichen Einrichtung, der/die dann die Bögen im Haus weiterverschickte. Die Fragebögen waren für das Auskunfts- und Informationspersonal der Institutionen bestimmt. Auszufüllen war der Fragebogen entweder alleine, durch mehrere Personen oder durch die ganze Abteilung. Als Zeitraum waren vier Wochen veranschlagt, jedoch kamen fast alle Bögen sehr schnell, meist schon nach wenigen Tagen, zurück. 
Von acht Institutionen antworteten insgesamt sieben. Von den sieben Einrichtungen antworteten mit einem gemeinsamen Fragebogen drei Institutionen und vier Institutionen antworteten mit bis zu fünf Antwortbögen. So bildeten insgesamt 18 ausgefüllte Fragebögen die Grundlage für die Auswertung.

Inhaltlich war die Vorgabe, dass die Fragebögen von den Mitarbeitern der Einrichtungen bewusst subjektiv, mit eigenen Erfahrungen aus der Praxis, beantwortet werden sollten. Die Fragen zielten direkt auf die known-item search ab und sollten das Rechercheverhalten mit dieser Art von Suche im Alltag in Form von Schätzwerten wiederspiegeln.

\subsection{Ergebnisse der Befragung}

In diesem Kapitel werden die Ergebnisse der Umfrage in der Reihenfolge ihres Auftretens präsentiert.

Laut Einschätzung der Mitarbeiter recherchieren 55\% der Nutzer bei einer Suche mit der known-item search. Das heißt im Umkehrschluss, dass 45\% aller Nutzer mit einer thematischen Suche recherchieren.

Das Informationspersonal sucht nach eigenen Aussagen zu 66\% mit einer knownitem search im Auskunftsdienst und zu 34\% mit einer thematischen Suche. Hierbei gibt es aber starke Abweichungen unter den einzelnen Antworten. So sind bei diesem Punkt Angaben bei der known-item search zu finden, die von $50 \%$ bis zu $90 \%$ reichen.

Am häufigsten, bei Nutzern und Personal, findet eine Recherche nach Titel beziehungsweise Titelstichwörter und Verfasser statt. Auch wird in einigen Institutionen häufiger mit Jahreszahlen recherchiert. Die Recherchen mittels ISBN beziehungsweise Signatur sind sehr schwach vertreten, wobei nach einer ISBN noch häufiger recherchiert wird als nach einer Signatur. Es war zudem eine Aussage in den Fragebögen zu finden, wonach nur Bibliothekare mittels Signatur recherchieren würden. 
Nach Einschätzung der Befragten wird in der erweiterten Suche zu 70\% mit einer kombinierten Suche recherchiert. Bei der Suche mittels Kombination dominiert eindeutig die Verknüpfung Titel- und Verfasser. Es gibt Institutionen, die ihre Nutzer für diese Kombinationsart schulen, um die Treffermenge gering zu halten. Öfters wurde auch eine Recherche mittels Kombination Titel und Jahr als typisch genannt.

Fast immer wird die einfache Suche bevorzugt. Zu insgesamt 73\% wird die Recherche hierüber getätigt. Für die erweiterte Suche entfällt daher eine Nutzung von $27 \%$. Einige Bibliotheken gaben allerdings an, dass sie für ihre OPACs standardmäßig nur die erweiterte Suche anbieten, zumindest bei den Rechercheterminals in der Bibliothek.

Für das Auftreten von Null-Treffern gaben die bei der Umfrage beteiligten Bibliotheken an, dass zu viel eingegeben wurde (gemeint sind hier zum Beispiel Wörter), falsche Suchfelder benutzt wurden oder Tippfehler, zu denen auch falsche Singularoder Pluralformen gehören. Weiterhin liegen Fehlerquellen oftmals bei der falschen Interpretation eines Zitats, zum Beispiel wird ein Aufsatz als Monographie gesucht, oder einer nicht normierten Eingabe, wie dies im Suchfeld für den Verfasser geschehen kann. Ungenaue Eingaben, beispielsweise bei Signaturen und unbekannte Möglichkeiten wie die Trunkierung sind ebenfalls Gründe, die öfters zu Null-Treffern führen. Genannt, aber nicht näher ausgeführt in den Fragebögen, wurden zudem die Verwechslung der Medienform und die Benutzung von Abkürzungen.

Verhaltensmuster, die Nutzer bei einer Nulltrefferanzeige zu Tage legen, ähneln sich laut Aussagen der befragten Bibliotheken sehr. So versuchen die allermeisten Nutzer ihr Glück mit einer erneuten Recherche, die mit veränderten Eingaben beziehungsweise anderen Kombinationen stattfindet. Führen geänderte Rechercheanfragen nicht zum Ziel, so wird oft bei der Auskunft nachgefragt. Ein gewisser Anteil der Nutzer zeigt allerdings eine Resignation, wie 'das Buch gibt es nicht'. Eher gering ist die Durchführung von Fernleihbestellungen bei einer negativen Recherche. Dies tätigen, laut Anmerkung in den Fragebögen, in der Regel nur erfahrene Nutzer.

Interessanterweise gibt es einige auffällige Vorgehensweisen von Nutzern bei einer known-item search, die immer wieder auftreten. So werden zum Beispiel Aufsätze als 
Monographien gesucht, oder die Eingabe eines kompletten Literaturzitates mit allen Füllwörtern. Hier liegt die Vermutung von 'copy and paste' nahe. Weitere wichtige Punkte, die genannt wurden, sind die Eingabe aller Satzzeichen, sowie Groß- und Kleinschreibung (die bei einer Recherche aber unproblematisch ist), die Eingabe von im Katalog nicht berücksichtigten Personen wie beispielsweise einem zweiten Herausgeber. In einigen Fällen wurde noch eine seltene Nutzung der Suchfelder ISBN, Verlag oder Körperschaft und die wenig genutzten Sucheinschränkungen genannt. Es wurde zudem angemerkt, dass die Mentalität, alles in die einfache Suche zu schreiben anstatt in bekannte Felder der erweiterten Suche, auch zu einem typischen Verhalten der Nutzer gehört.

Genannt, aber nicht näher beschrieben, wurde darüber hinaus noch die Suche mit nicht aufgelösten Abkürzungen.

Zuletzt wurde gefragt, ob die befragten Personen etwas an ihrem Katalog verbessern möchten. Meist wurde hierauf, ohne ausführliche Erklärung, geantwortet, dass man die Funktion der freien Suche verbessern könnte, eine Verfassersuche mit zwei Vornamen günstig wäre oder der Verfasser auch in der Form Vorname Nachname eingegeben werden kann. Es wird aber auf der anderen Seite auch eine Reduktion der Suchmöglichkeiten gefordert, ein Drop-down Menü sowie ein Auffangen von falschen Schreibweisen in der Form 'Meinten Sie vielleicht...?'.

\subsection{Rückschlüsse und Vergleich mit den Logfiles}

Es ist sofort zu erkennen, dass sehr viele Ergebnisse der Logfileuntersuchung mit den Erkenntnissen aus den Fragebögen und somit aus der Praxis übereinstimmen. Es bestätigt sich, dass Nutzer bei einer known-item search am allermeisten nach Titel beziehungsweise Titelstichwörtern und Verfasser recherchieren. Die geringe Recherche nach Signaturen und ISBN deckt sich ebenfalls mit den Aussagen aus der Praxis.

Ist wie beim LBZ Rheinland-Pfalz im eigentlichen Sinne nur die erweiterte Suche existent, so recherchieren hierüber auch mehr Nutzer als über die einfache Suche. In 
der Praxis wird aber größtenteils, auf Grund des Angebots der Bibliotheken, über die einfache Suche recherchiert, wie die Logfiles der UB Heidelberg bestätigen.

Führen Nutzer eine kombinierte Suche durch, wird in der Regel mit dem Booleschen Operator UND Verfasser und Titel gesucht. Nicht so häufig waren bei den betrachteten Logfiles Kombinationen aus Titel und Jahr, die aber laut Praxiserfahrungen in einigen Institutionen ebenfalls öfters vorkommen.

Gründe wie 'zu-viel-eingegeben', Wahl von falschen Suchfeldern, Tippfehler, falsche Interpretationen von Zitaten wie zum Beispiel Aufsatzartikel, ungenaue Eingaben und die Benutzung von Abkürzungen stellen Fehlerursachen dar, die sich sowohl in den Logfiles wie auch den Ergebnissen der Fragebögen wiederspiegeln.

Eine aus der Praxis stammende Fehlerquelle, nämlich nicht normierte Eingaben wie zum Beispiel Nachname Komma Vorname im Suchfeld für Verfasser, lässt sich bei den Logfiles zwar auch feststellen, ist hier aber kein Grund für ein negatives Rechercheergebnis (Bsp. 4.2.3).

Die Anmerkungen aus der Praxis über die für sehr viele Nutzer unbekannten Möglichkeiten wie weitere Boolesche Operatoren oder die Trunkierungsmöglichkeiten lassen sich eindeutig durch die Logfiles bestätigen.

Bei einer Nulltrefferanzeige versuchen es Nutzer meist, so auch die Aussagen aus den Fragebögen, mit einer erneuten Recherche. Dies deckt sich exakt mit den Feststellungen der Logfiles (Bsp. 4.2.25 - 4.2.27).

Verbesserungsmöglichkeiten werden dagegen sehr individuell und oft institutionsabhängig genannt. Jedoch hätten sehr viele Einrichtungen gerne Programme zum Auffangen von falschen Schreibweisen oder eine Vereinfachung der Recherche beziehungsweise Benutzung. 


\section{Fazit}

Logfile-Untersuchungen in Bibliotheken oder vergleichbaren Einrichtungen sind nichts Neues. Schon seit Aufkommen dieser Methode sind Institutionen bestrebt, bezüglich ihrer Dienstleistungen oder Statistiken eine solche Untersuchung anzuwenden. Die Erwartungen und Bedürfnisse, die an eine Logfileuntersuchung gestellt werden, sind sehr unterschiedlich, und daher nicht einfach zusammenfassbar. Neben anderen Methoden wie Befragungen oder ähnliches kann sie immer einen unverfälschten Blick auf die Eingabe und Suche im OPAC bieten. Da Rückfragen nicht möglich sind, liegen Rückschlüsse daher allein bei der auswertenden Person. Eine Besonderheit dieser Arbeit ist, dass die known-item search in der Literatur noch nie so tief und ausführlich behandelt wurde.

Diese Untersuchung erbrachte folgende Erkenntnisse:

Bibliotheksnutzer wissen sehr oft und vor allem auch ganz genau, was sie wollen. Jedoch steht in einigen Fällen der OPAC zwischen dem Nutzer und dem gewünschten Medium. Auch wenn ein Großteil der Nutzer das Instrumentarium OPAC beherrscht, so haben mindestens genauso viele Nutzer oftmals größere oder teilweise auch unüberwindbare Probleme, ihre Recherchen erfolgreich durchzuführen.

Die aus dieser Untersuchung gewonnenen Ergebnisse mögen nicht auf jede Bibliothek zutreffen, da unterschiedliche Bibliotheken selbstverständlich unterschiedliche Bedürfnisse haben. So ist jedoch klar, dass die known-item search noch weiter verbessert werden kann, denn der Nutzer weiß bereits, was er möchte und Bibliotheken sollten inn deshalb als Dienstleister im Informationssektor bestmöglichst unterstützen. Es ist folglich nur ratsam, mit den heutigen Mitteln, sowohl technisch als auch finanziell, den OPAC als zentralen Recherchepunkt der Bibliothek zu optimieren. Dabei liegt es auch ein Stück weit am Bibliothekspersonal, ihre Sichtweise zu ändern und die angebotenen Dienstleistungen aus Kundensicht zu betrachten, um ihren Nutzern entgegenzukommen.

Die Recherche, sei es über die einfache Suche oder die erweiterte Suche, muss unbedingt so einfach, selbsterklärend und effizient wie möglich gehalten werden. Hilfetexte und Nutzerschulungen werden von Nutzern kaum wahrgenommen und dürfen nur das letzte Mittel in einer langen Liste von technischen Möglichkeiten sein. 
Vor allem 'kleinere' Verbesserungen wie eine effektive Indexierung, um beispielsweise zwei Verfasser in einem Feld suchbar zu machen, oder eine automatische Trunkierung, die vor allem bei der einfachen Suche Eingaben soweit kürzt, bis das System einen 'passenden' Eintrag findet, sind hierfür Beispiele.

Unabdingbar ist, wie es zum Beispiel HEIDI schon propagiert, eine stärke Orientierung an der Suchmaschinentechnologie. Automatische Rechtschreibkorrektur, automatische Vervollständigung bei der Eingabe oder eine Fuzzy-Suche wie im OPAC der Staatsbibliothek zu Berlin sind durchführbare Verbesserungen, die der immer stärker werdenden 'Google-Mentalität' entgegenkommen.

Die known-item search wird daher auch weiterhin ein spannendes Feld darstellen. Dabei hat jede Bibliothek die Möglichkeit, eine eigene Logfileuntersuchung mit ihren Daten durchzuführen und die daraus gewonnenen Erkenntnisse zur Verbesserung ihres OPACs zu nutzen. Aus diesem Grund sollte kein Aufwand gescheut werden, einen selbsterklärenden und einfach bedienbaren OPAC anzubieten. Schließlich ist der Nutzer die Legitimationsberechtigung einer jeden Bibliothek. 


\section{Anhang A: Original Logfiles}

Im Folgenden werden einzelne Ausschnitte aus den Logfiles dargestellt, so wie sie die Bibliotheken aus ihren Systemen erzeugt haben.

\section{A.1a: Logfiles des LBZ Rheinland-Pfalz vom 18.10.2011}

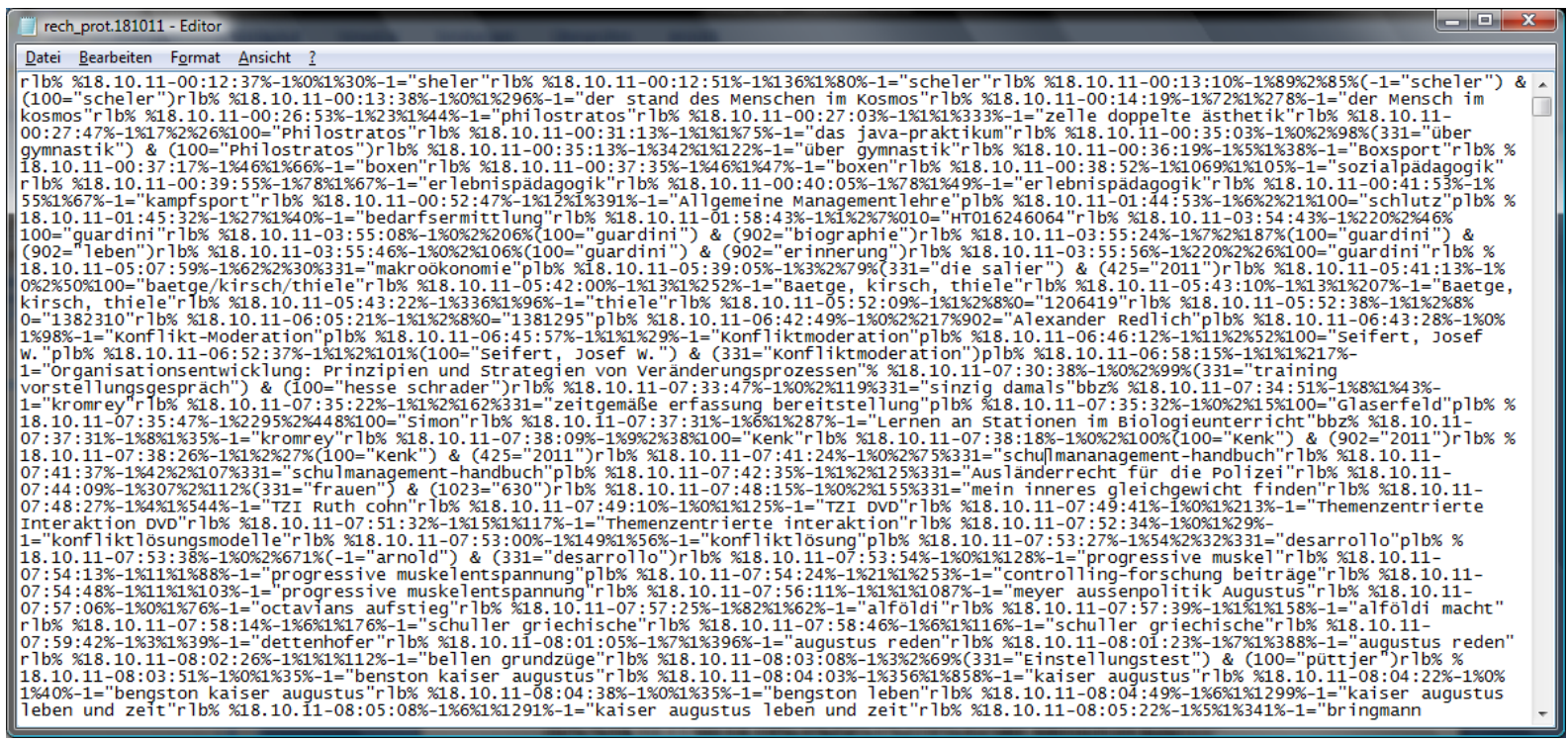

Abb. 12: Abruf der Logfiles vom 18.10.2011 mittels Editor

Ein vergrößerter Ausschnitt der aus dem Editor kopierten Logfiles:

rlb\% \%18.10.11-00:12:37\%-1\%0\%1\%30\%-1="sheler"

rlb\% \%18.10.11-00:12:51\%-1\%136\%1\%80\%-1="scheler"

rlb\% \%18.10.11-00:13:10\%-1\%89\%2\%85\%(-1="scheler") \& (100="scheler")

rlb\% \%18.10.11-00:13:38\%-1\%0\%1\%296\%-1="der stand des Menschen im Kosmos"

rlb\% \%18.10.11-00:14:19\%-1\%72\%1\%278\%-1="der Mensch im kosmos"

rlb\% \%18.10.11-00:26:53\%-1\%23\%1\%44\%-1="philostratos"

rlb\% \%18.10.11-00:27:03\%-1\%1\%1\%333\%-1="zelle doppelte ästhetik"

rlb\% \%18.10.11-00:27:47\%-1\%17\%2\%26\%100="Philostratos"

rlb\% \%18.10.11-00:31:13\%-1\%1\%1\%75\%-1="das java-praktikum" 
rlb\% \%18.10.11-00:35:03\%-1\%0\%2\%98\%(331="über gymnastik") \& (100="Philostratos")

rlb\% \%18.10.11-00:35:13\%-1\%342\%1\%122\%-1="über gymnastik" rlb\% \%18.10.11-00:36:19\%-1\%5\%1\%38\%-1="Boxsport" rlb\% \%18.10.11-00:37:17\%-1\%46\%1\%66\%-1="boxen" rlb\% \%18.10.11-00:37:35\%-1\%46\%1\%47\%-1="boxen" rlb\% \%18.10.11-00:38:52\%-1\%1069\%1\%105\%-1="sozialpädagogik" rlb\% \%18.10.11-00:39:55\%-1\%78\%1\%67\%-1="erlebnispädagogik" rlb\% \%18.10.11-00:40:05\%-1\%78\%1\%49\%-1="erlebnispädagogik" rlb\% \%18.10.11-00:41:53\%-1\%55\%1\%67\%-1="kampfsport" rlb\% \%18.10.11-00:52:47\%-1\%12\%1\%391\%-1="Allgemeine Managementlehre" plb\% \%18.10.11-01:44:53\%-1\%6\%2\%21\%100="schlutz" plb\% \%18.10.11-01:45:32\%-1\%27\%1\%40\%-1="bedarfsermittlung" rlb\% \%18.10.11-01:58:43\%-1\%1\%2\%7\%010="HT016246064" rlb\% \%18.10.11-03:54:43\%-1\%220\%2\%46\%100="guardini" rlb\% \%18.10.11-03:55:08\%-1\%0\%2\%206\%(100="guardini") \& (902="biographie") rlb\% \%18.10.11-03:55:24\%-1\%7\%2\%187\%(100="guardini") \& (902="leben") rlb\% \%18.10.11-03:55:46\%-1\%0\%2\%106\%(100="guardini") \& (902="erinnerung") rlb\% \%18.10.11-03:55:56\%-1\%220\%2\%26\%100="guardini" rlb\% \%18.10.11-05:07:59\%-1\%62\%2\%30\%331="makroökonomie" plb\% \%18.10.11-05:39:05\%-1\%3\%2\%79\%(331="die salier") \& (425="2011") rlb\% \%18.10.11-05:41:13\%-1\%0\%2\%50\%100="baetge/kirsch/thiele" rlb\% \%18.10.11-05:42:00\%-1\%13\%1\%252\%-1="Baetge, kirsch, thiele" rlb\% \%18.10.11-05:43:10\%-1\%13\%1\%207\%-1="Baetge, kirsch, thiele" rlb\% \%18.10.11-05:43:22\%-1\%336\%1\%96\%-1="thiele" rlb\% \%18.10.11-05:52:09\%-1\%1\%2\%8\%0="1206419" rlb\% \%18.10.11-05:52:38\%-1\%1\%2\%8\%0="1382310" rlb\% \%18.10.11-06:05:21\%-1\%1\%2\%8\%0="1381295" plb\% \%18.10.11-06:42:49\%-1\%0\%2\%217\%902="Alexander Redlich" plb\% \%18.10.11-06:43:28\%-1\%0\%1\%98\%-1="Konflikt-Moderation" plb\% \%18.10.11-06:45:57\%-1\%1\%1\%29\%-1="Konfliktmoderation" plb\% \%18.10.11-06:46:12\%-1\%11\%2\%52\%100="Seifert, Josef W." plb\% \%18.10.11-06:52:37\%-1\%1\%2\%101\%(100="Seifert, Josef W.") \& (331="Konfliktmoderation") 


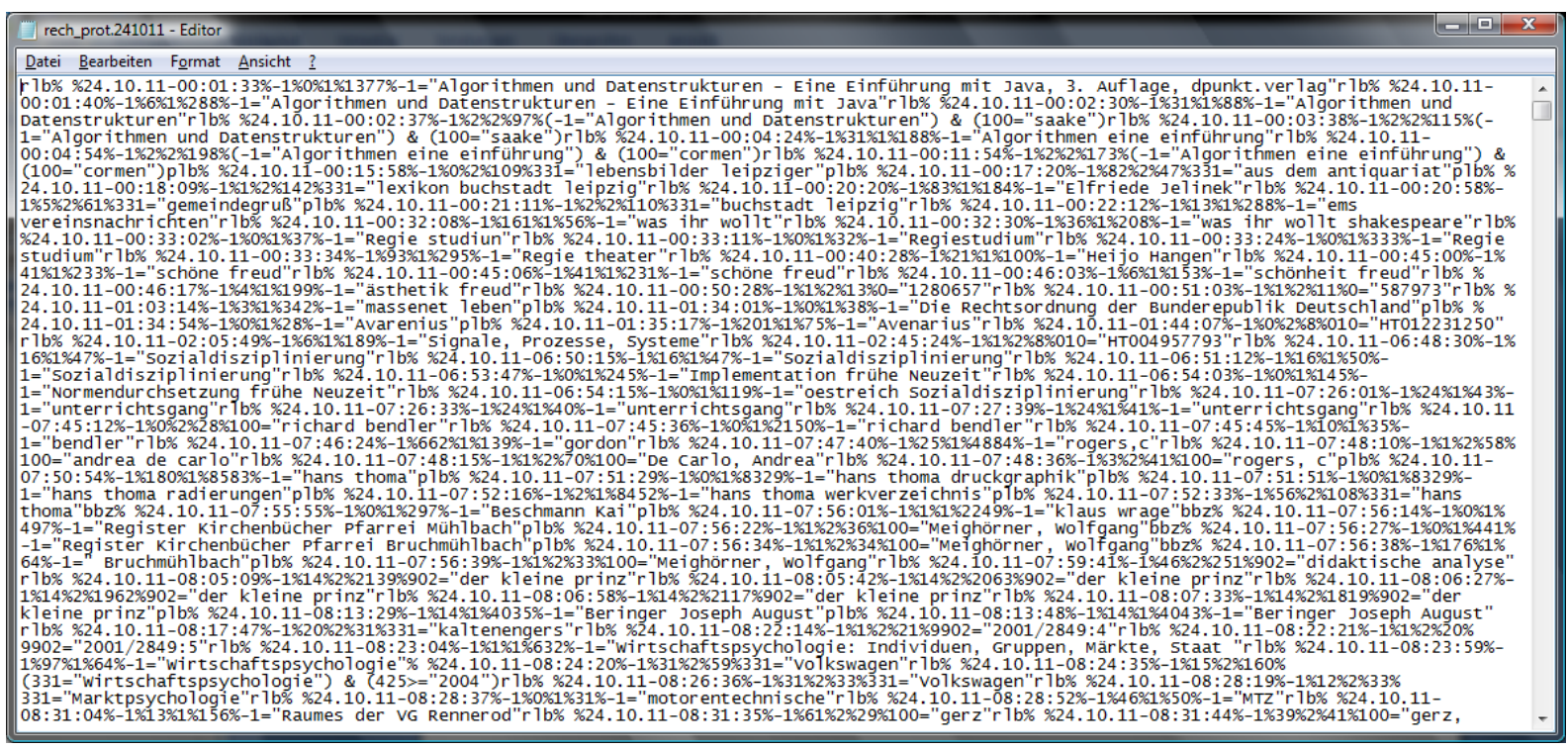

Abb. 13: Abruf der Logfiles vom 24.10.2011 mittels Editor

Ein vergrößerter Ausschnitt der aus dem Editor kopierten Logfiles:

rlb\% \%24.10.11-00:01:33\%-1\%0\%1\%1377\%-1="Algorithmen und Datenstrukturen Eine Einführung mit Java, 3. Auflage, dpunkt.verlag" rlb\% \%24.10.11-00:01:40\%-1\%6\%1\%288\%-1="Algorithmen und Datenstrukturen Eine Einführung mit Java" rlb\% \%24.10.11-00:02:30\%-1\%31\%1\%88\%-1="Algorithmen und Datenstrukturen" rlb\% \%24.10.11-00:02:37\%-1\%2\%2\%97\%(-1="Algorithmen und Datenstrukturen") \& (100="saake")

rlb\% \%24.10.11-00:03:38\%-1\%2\%2\%115\%(-1="Algorithmen und Datenstrukturen") \& $(100="$ saake") rlb\% \%24.10.11-00:04:24\%-1\%31\%1\%188\%-1="Algorithmen eine einführung" rlb\% \%24.10.11-00:04:54\%-1\%2\%2\%198\%(-1="Algorithmen eine einführung") \& (100="cormen") rlb\% \%24.10.11-00:11:54\%-1\%2\%2\%173\%(-1="Algorithmen eine einführung") \& (100="cormen") plb\% \%24.10.11-00:15:58\%-1\%0\%2\%109\%331="lebensbilder leipziger" plb\% \%24.10.11-00:17:20\%-1\%82\%2\%47\%331="aus dem antiquariat" plb\% \%24.10.11-00:18:09\%-1\%1\%2\%142\%331="lexikon buchstadt leipzig" 
rlb\% \%24.10.11-00:20:20\%-1\%83\%1\%184\%-1="Elfriede Jelinek" rlb\% \%24.10.11-00:20:58\%-1\%5\%2\%61\%331="gemeindegruß" plb\% \%24.10.11-00:21:11\%-1\%2\%2\%110\%331="buchstadt leipzig" rlb\% \%24.10.11-00:22:12\%-1\%13\%1\%288\%-1="ems vereinsnachrichten" rlb\% \%24.10.11-00:32:08\%-1\%161\%1\%56\%-1="was ihr wollt" rlb\% \%24.10.11-00:32:30\%-1\%36\%1\%208\%-1="was ihr wollt shakespeare" rlb\% \%24.10.11-00:33:02\%-1\%0\%1\%37\%-1="Regie studiun" rlb\% \%24.10.11-00:33:11\%-1\%0\%1\%32\%-1="Regiestudium" rlb\% \%24.10.11-00:33:24\%-1\%0\%1\%333\%-1="Regie studium" rlb\% \%24.10.11-00:33:34\%-1\%93\%1\%295\%-1="Regie theater" rlb\% \%24.10.11-00:40:28\%-1\%21\%1\%100\%-1="Heijo Hangen" rlb\% \%24.10.11-00:45:00\%-1\%41\%1\%233\%-1="schöne freud" rlb\% \%24.10.11-00:45:06\%-1\%41\%1\%231\%-1="schöne freud" rlb\% \%24.10.11-00:46:03\%-1\%6\%1\%153\%-1="schönheit freud" rlb\% \%24.10.11-00:46:17\%-1\%4\%1\%199\%-1="ästhetik freud" rlb\% \%24.10.11-00:50:28\%-1\%1\%2\%13\%0="1280657" rlb\% \%24.10.11-00:51:03\%-1\%1\%2\%11\%0="587973" rlb\% \%24.10.11-01:03:14\%-1\%3\%1\%342\%-1="massenet leben" plb\% \%24.10.11-01:34:01\%-1\%0\%1\%38\%-1="Die Rechtsordnung der Bunderepublik Deutschland" plb\% \%24.10.11-01:34:54\%-1\%0\%1\%28\%-1="Avarenius" plb\% \%24.10.11-01:35:17\%-1\%201\%1\%75\%-1="Avenarius" rlb\% \%24.10.11-01:44:07\%-1\%0\%2\%8\%010="HT012231250" rlb\% \%24.10.11-02:05:49\%-1\%6\%1\%189\%-1="Signale, Prozesse, Systeme" rlb\% \%24.10.11-02:45:24\%-1\%1\%2\%8\%010="HT004957793" rlb\% \%24.10.11-06:48:30\%-1\%16\%1\%47\%-1="Sozialdisziplinierung" rlb\% \%24.10.11-06:50:15\%-1\%16\%1\%47\%-1="Sozialdisziplinierung" rlb\% \%24.10.11-06:51:12\%-1\%16\%1\%50\%-1="Sozialdisziplinierung" rlb\% \%24.10.11-06:53:47\%-1\%0\%1\%245\%-1="Implementation frühe Neuzeit" rlb\% \%24.10.11-06:54:03\%-1\%0\%1\%145\%-1="Normendurchsetzung frühe Neuzeit" rlb\% \%24.10.11-06:54:15\%-1\%0\%1\%119\%-1="oestreich Sozialdisziplinierung" rlb\% \%24.10.11-07:26:01\%-1\%24\%1\%43\%-1="unterrichtsgang" rlb\% \%24.10.11-07:26:33\%-1\%24\%1\%40\%-1="unterrichtsgang" 


\section{A.1c: Logfiles der UB Heidelberg vom 26.10.2011}

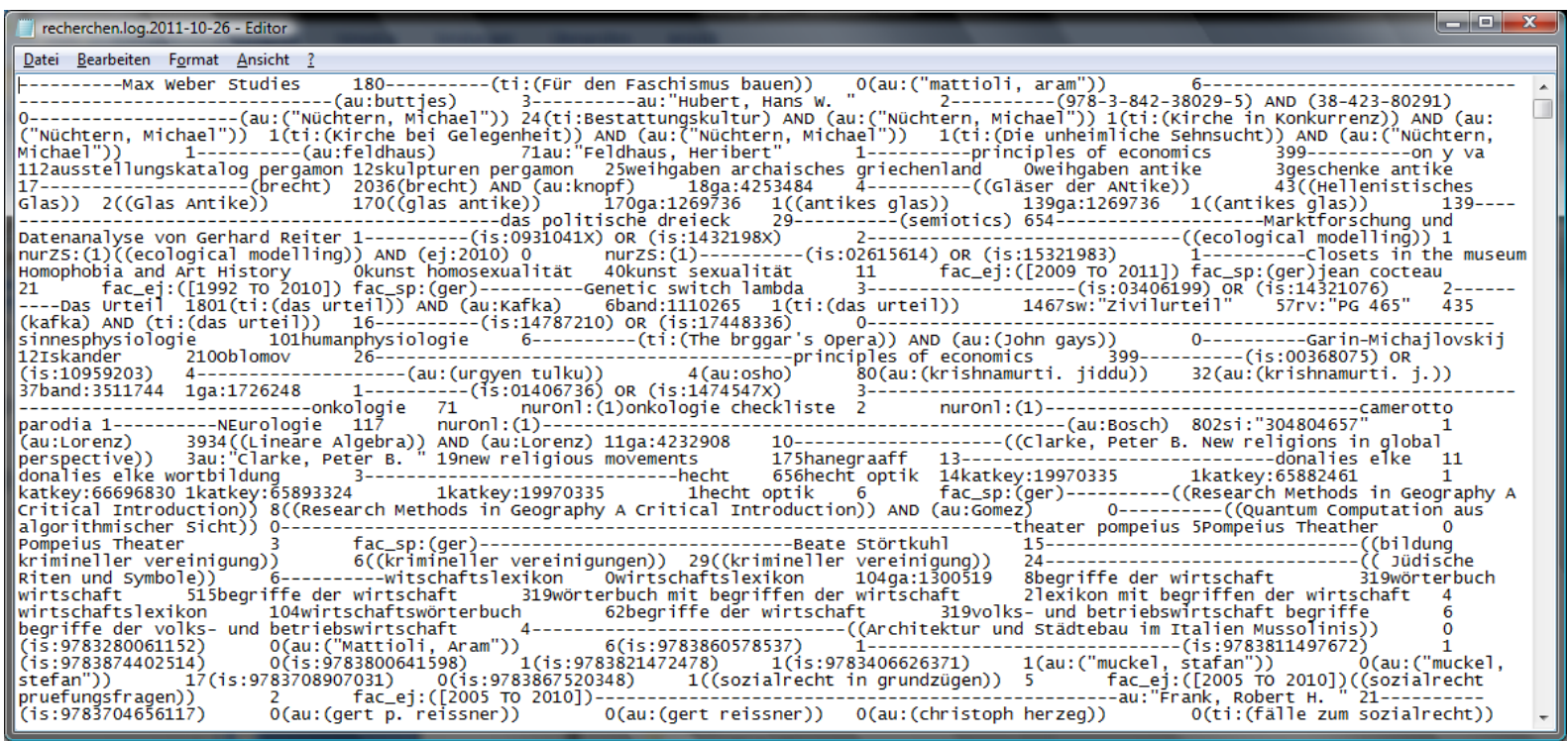

Abb. 14: Abruf der Logfiles vom 26.10.2011 mittels Editor

Ein vergrößerter Ausschnitt der aus dem Editor kopierten Logfiles mit Sessiongrenze:

Max Weber Studies 180

(ti:(Für den Faschismus bauen)) 0

(au:("mattioli, aram")) 6

6

(au:buttjes) 3

au:"Hubert, Hans W. " 2

(978-3-842-38029-5) AND (38-423-80291) 0 
(au:("Nüchtern, Michael")) 24

(ti:Bestattungskultur) AND (au:("Nüchtern, Michael")) 1

(ti:(Kirche in Konkurrenz)) AND (au:("Nüchtern, Michael")) 1

(ti:(Kirche bei Gelegenheit)) AND (au:("Nüchtern, Michael")) 1

(ti:(Die unheimliche Sehnsucht)) AND (au:("Nüchtern, Michael")) 1

(au:feldhaus) 71

au:"Feldhaus, Heribert" 1

principles of economics 399

on y va 112

ausstellungskatalog pergamon 12

skulpturen pergamon $\quad 25$

weihgaben archaisches griechenland 0

weihgaben antike 3

geschenke antike 17

(brecht) 2036

(brecht) AND (au:knopf) 18

ga:4253484 4

((Gläser der ANtike)) 43

((Hellenistisches Glas)) 2

((Glas Antike)) 170

((glas antike)) 170

ga:1269736 1

((antikes glas)) 139

ga:1269736 1

((antikes glas)) 139 


\section{A.1d: Logfiles der UB Heidelberg vom 05.11.2011}

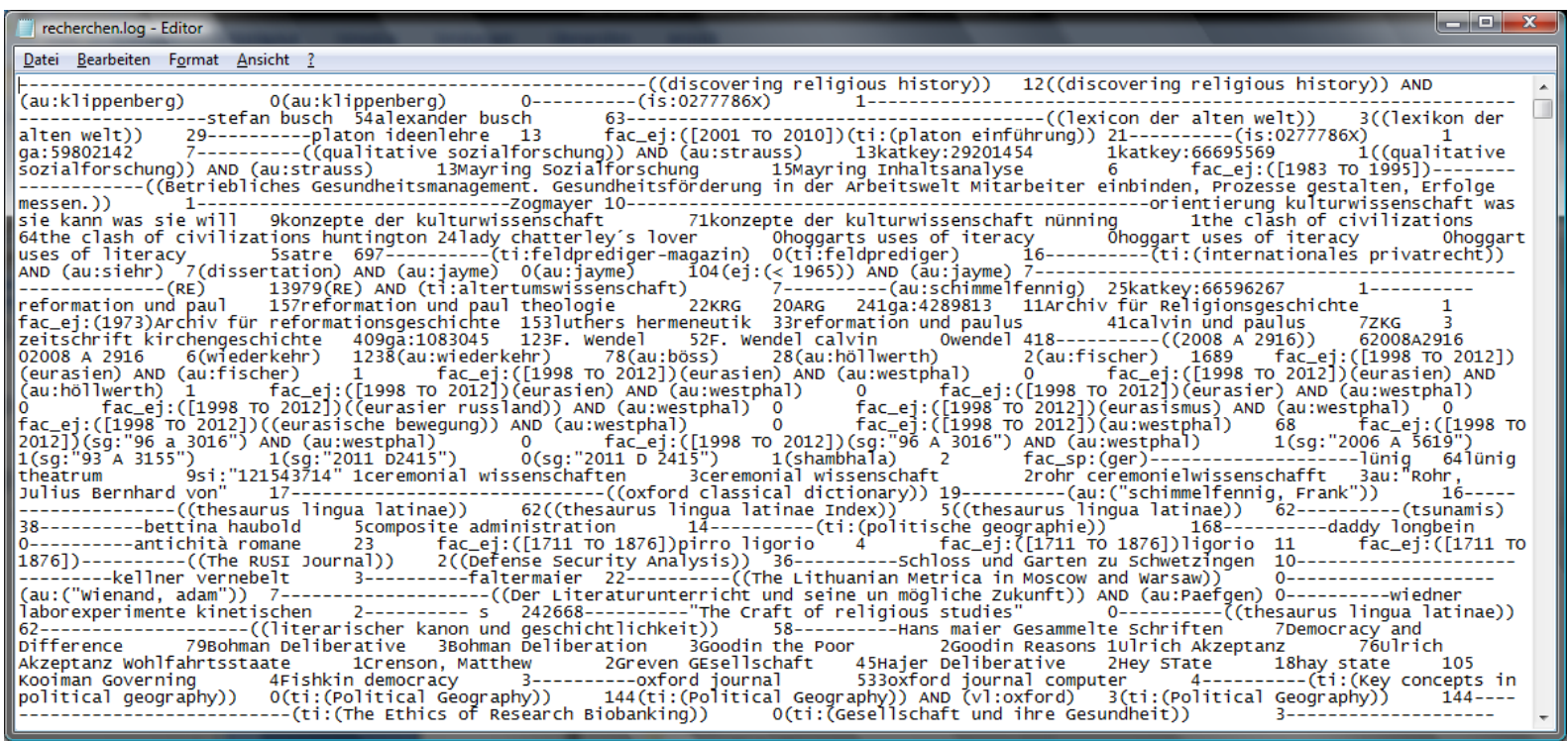

Abb. 15: Abruf der Logfiles vom 05.11.2011 mittels Editor

Ein vergrößerter Ausschnitt der aus dem Editor kopierten Logfiles mit Sessiongrenze:

((discovering religious history)) 12

((discovering religious history)) AND (au:klippenberg) 0

(au:klippenberg) 0

(is:0277786X) 1 
stefan busch 54

alexander busch

63

((lexicon der alten welt)) 3

((lexikon der alten welt)) 29

platon ideenlehre 13 fac_ej:([2001 TO 2010])

(ti:(platon einführung)) 21

(is:0277786X) 1

ga:598021427

((qualitative sozialforschung)) AND (au:strauss)

katkey:29201454 1

katkey:66695569 1

((qualitative sozialforschung)) AND (au:strauss) 13

Mayring Sozialforschung 15

Mayring Inhaltsanalyse $6 \quad$ fac_ej:([1983 TO 1995])

((Betriebliches Gesundheitsmanagement. Gesundheitsförderung in der Arbeitswelt Mitarbeiter einbinden, Prozesse gestalten, Erfolge messen.))

Zogmayer 10 


\section{Anhang B: Umgewandelte Logfiles}

Die Beispiele in diesem Teil des Anhangs sind identisch mit den Beispielen aus Anhang A. Hier sind jedoch die Logfiles bereits umgewandelt und dadurch besser lesbar.

\section{B.1a: Umgewandelte Logfiles des LBZ Rheinland-Pfalz vom 18.10.2011}

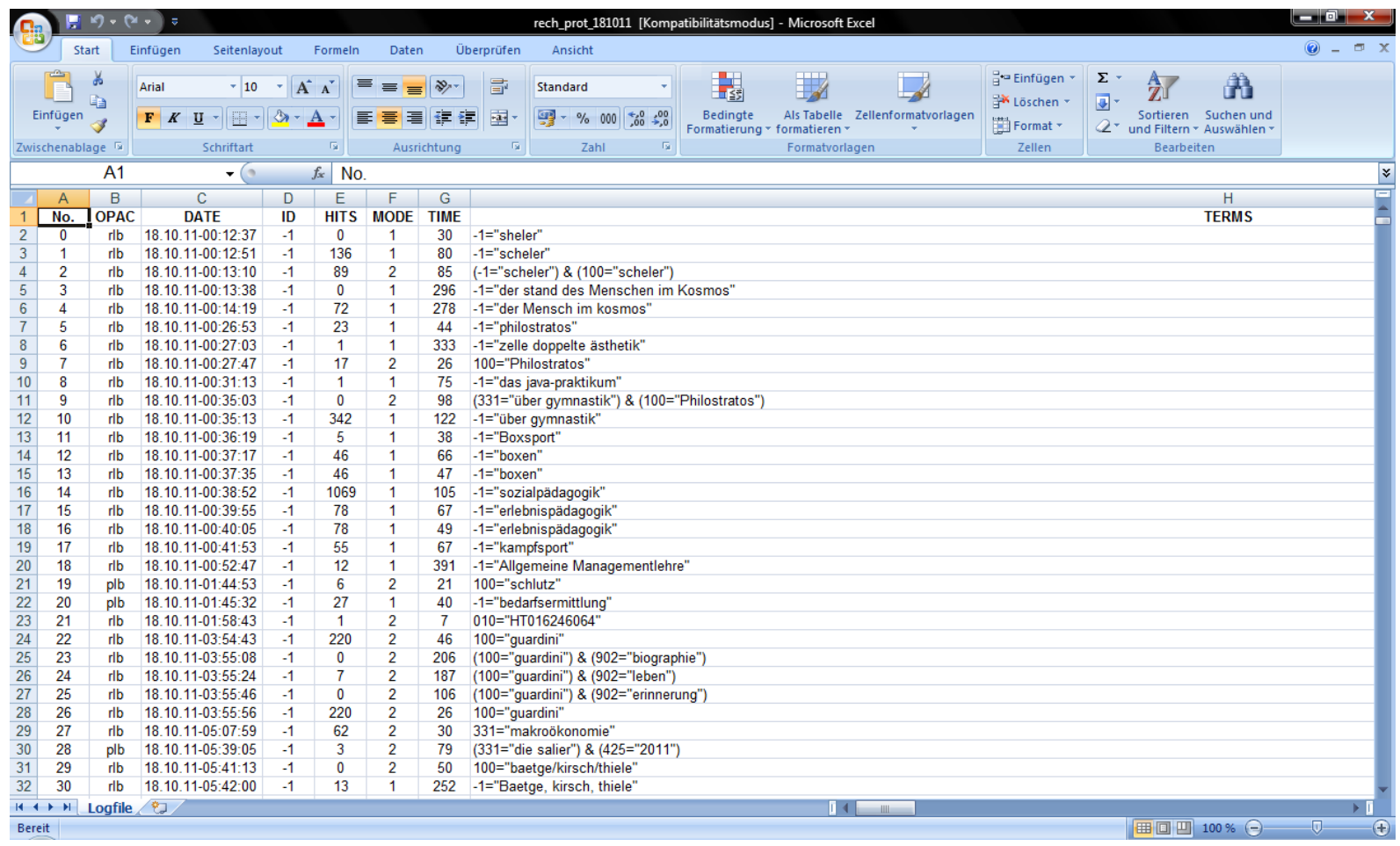

Abb. 16: Logfiles vom 18.10.2011 dargestellt mittels Excel

Ein vergrößerter Ausschnitt der aus Excel stammenden Logfiles:

No. OPACDATE ID HITS MODE TIME TERMS

$\begin{array}{llllllll}0 & \text { rlb } & 18.10 .11-00: 12: 37 & -1 & 0 & 1 & 30 & -1=" s h e l e r " \\ 1 & \text { rlb } & 18.10 .11-00: 12: 51 & -1 & 136 & 1 & 80 & -1=" s c h e l e r " \\ 2 & \text { rlb } & 18.10 .11-00: 13: 10 & -1 & 89 & 2 & 85 & (-1=" s c h e l e r ") \& \\ & (100=\text { "scheler") } & & & & & \end{array}$


$3 \quad$ rlb 18.10.11-00:13:38 $-1 \quad 0 \quad 1 \quad 296 \quad-1="$ "der stand des Menschen im Kosmos"

$4 \quad$ rlb 18.10.11-00:14:19 $-1 \quad 72 \quad 1 \quad 278$-1="der Mensch im kosmos"

$5 \quad \mathrm{rlb}$

18.10.11-00:26:53 $-1 \quad 23 \quad 1$

$44-1=$ "philostratos"

$6 \quad \mathrm{rlb}$

18.10.11-00:27:03 $-1 \quad 1 \quad 1$

$333-1=$ ="zelle doppelte ästhetik"

$7 \quad \mathrm{rlb}$

18.10.11-00:27:47 -1

$17 \quad 2$

26

$8 \quad \mathrm{rlb}$

18.10.11-00:31:13 -1

1

$9 \mathrm{rlb}$

18.10.11-00:35:03 -1

\& $(100=" P h i l o s t r a t o s ")$

10 rlb

18.10.11-00:35:13 -1

$342 \quad 1$

122

11 rlb

18.10.11-00:36:19 -1

12 rlb

18.10.11-00:37:17 -1

$13 \mathrm{rlb}$

18.10.11-00:37:35 -1

$14 \quad \mathrm{rlb}$

18.10.11-00:38:52 -1

5

46

1

38

66

$15 \mathrm{rlb}$

18.10.11-00:39:55 -

16 rlb

18.10.11-00:40:05 -1

17 rlb

18.10.11-00:41:53 -1

$46 \quad 1$

47

10691

105

6

$100=" P h i l o s t r a t o s "$

75

-1="das java-praktikum"

$18 \quad \mathrm{rlb}$

18.10.11-00:52:47 -1 gementlehre"

19
plb
18.10 .11

20 plb

18.10.11-01:45:32 -1

$\begin{array}{ll}78 & 1\end{array}$

67

$49-1=$ =erlebnispädagogik"

$21 \mathrm{rlb}$

18.10.11-01:58:43 -1

78

$55 \quad 1$

$67-1=$ ="kampfsport"

121

391 -1="Allgemeine Mana-

22 rlb

18.10.11-03:54:43 -1

6

2

\section{1}

$100=$ "schlutz"

$23 \mathrm{rlb}$

18.10.11-03:55:08 -1 (902="biographie")

$24 \quad \mathrm{rlb} \quad 18.10 .11-03: 55: 24 \quad-1 \quad 7 \quad 2 \quad 187 \quad$ (100="guardini") \& (902="leben")

25 rlb 18.10.11-03:55:46 $-1 \quad 0 \quad 2 \quad 106 \quad$ (100="guardini") \& (902="erinnerung")

$26 \quad r l b \quad 18.10 .11-03: 55: 56 \quad-1 \quad 220 \quad 2 \quad 26 \quad 100="$ guardini"

27 rlb 18.10.11-05:07:59 $-1 \quad 62 \quad 2 \quad 30 \quad 331=$ "makroökonomie" 


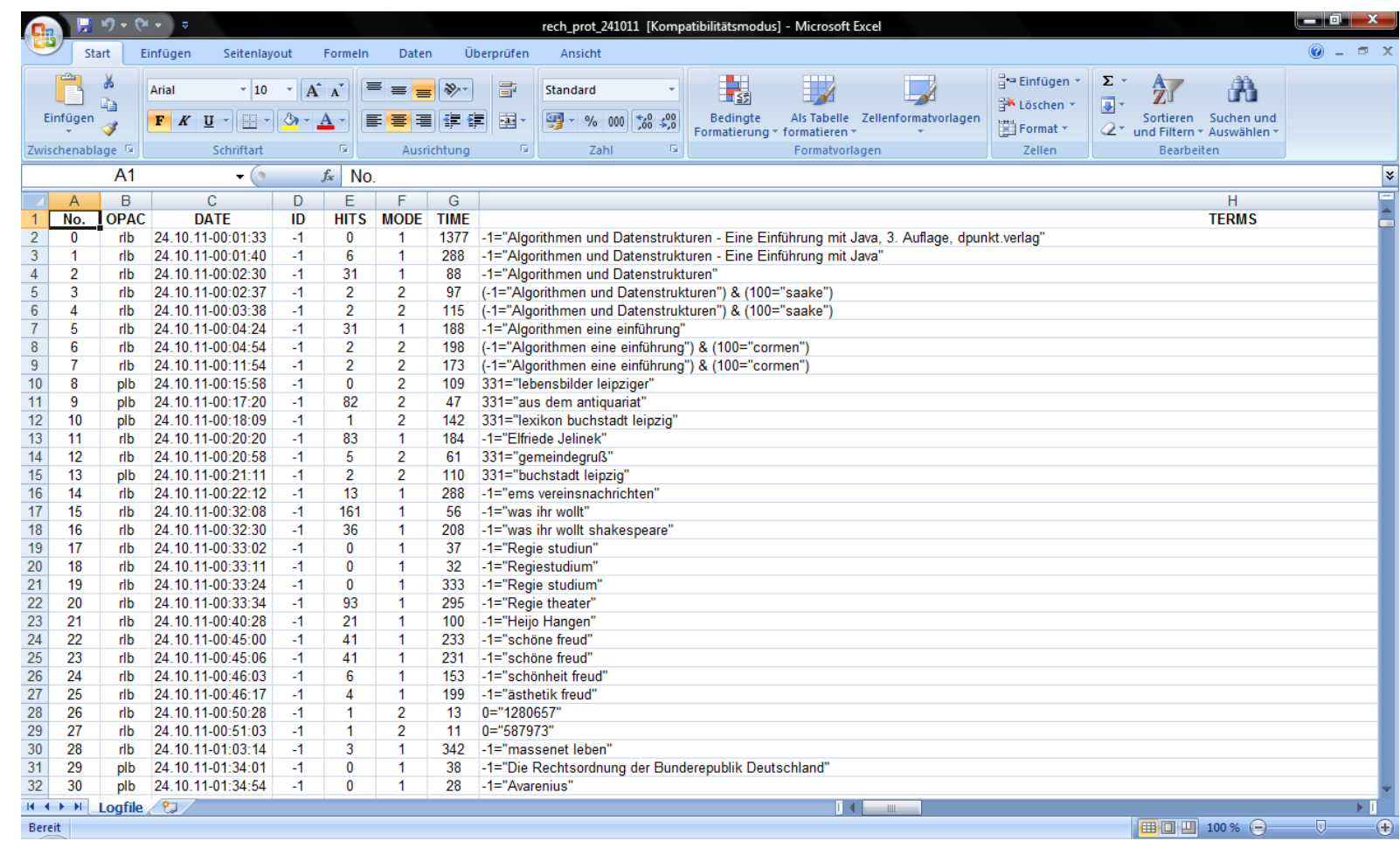

Abb. 17: Logfiles vom 24.10.2011 dargestellt mittels Excel

Ein vergrößerter Ausschnitt der aus Excel stammenden Logfiles:

No. OPACDATE ID HITS MODE TIME TERMS

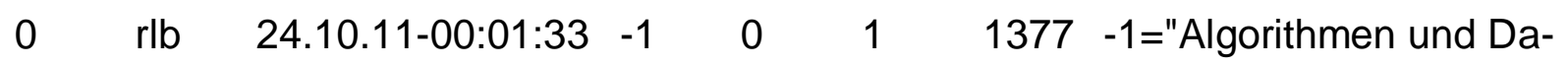
tenstrukturen - Eine Einführung mit Java, 3. Auflage, dpunkt.verlag"

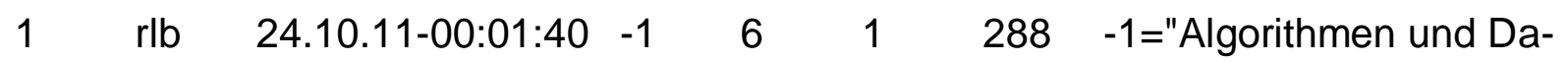
tenstrukturen - Eine Einführung mit Java"

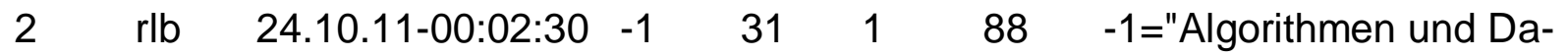
tenstrukturen"

$3 \quad \mathrm{rlb} \quad 24.10 .11-00: 02: 37 \quad-1 \quad 2 \quad 2 \quad 97 \quad$ (-1="Algorithmen und Datenstrukturen") \& (100="saake")

$4 \quad$ rlb 24.10.11-00:03:38 $-1 \quad 2 \quad 2 \quad 115$ (-1="Algorithmen und Datenstrukturen") \& (100="saake")

$5 \quad$ rlb 24.10.11-00:04:24 $-1 \quad 31 \quad 1 \quad 188$-1="Algorithmen eine einführung" 
$6 \quad \mathrm{rlb} \quad 24.10 .11-00: 04: 54 \quad-1 \quad 2 \quad 2 \quad 198 \quad$ (-1="Algorithmen eine einführung") \& (100="cormen")

$7 \quad \mathrm{rlb} \quad 24.10 .11-00: 11: 54 \quad-1 \quad 2 \quad 2 \quad 173 \quad$ (-1="Algorithmen eine einführung") \& (100="cormen")

$8 \quad$ plb 24.10.11-00:15:58 $-1 \quad 0 \quad 2 \quad 109$ 331="lebensbilder leipziger"

9 plb 24.10.11-00:17:20 $\quad-1 \quad 82 \quad 2 \quad 47 \quad 331=$ "aus dem antiquariat"

10 plb 24.10.11-00:18:09 $-1 \quad 1 \quad 2 \quad 142$ 331="lexikon buchstadt leipzig"

11 rlb 24.10.11-00:20:20 $\quad-1 \quad 83 \quad 1 \quad 184 \quad-1=$ "Elfriede Jelinek"

12 rlb 24.10.11-00:20:58 $-1 \quad 5 \quad 2 \quad 61$ 331="gemeindegruß"

13 plb 24.10.11-00:21:11 $-1 \quad 2 \quad 2 \quad 110$ 331="buchstadt leipzig"

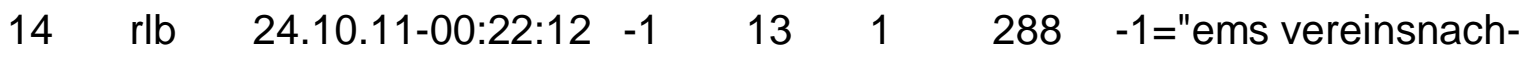
richten"

15 rlb 24.10.11-00:32:08 $-1 \quad 161 \quad 1 \quad 56 \quad-1=$ "was ihr wollt"

16 rlb 24.10.11-00:32:30 $-1 \quad 36 \quad 1 \quad 208$-1="was ihr wollt shakespeare"

17 rlb 24.10.11-00:33:02 $-1 \quad 0 \quad 1 \quad 37 \quad-1=$ "Regie studiun"

18 rlb 24.10.11-00:33:11 $-1 \quad 0 \quad 1 \quad 32 \quad-1=" R e g i e s t u d i u m "$

$19 \quad$ rlb 24.10.11-00:33:24 $-1 \quad 0 \quad 1 \quad 333 \quad-1="$ Regie studium"

20 rlb 24.10.11-00:33:34 $-1 \quad 93 \quad 1 \quad 295 \quad-1="$ "Regie theater"

21 rlb 24.10.11-00:40:28 $-1 \quad 21 \quad 1 \quad 100$-1="Heijo Hangen"

22 rlb 24.10.11-00:45:00 $-1 \quad 41 \quad 1 \quad 233 \quad-1=$ "schöne freud"

23 rlb 24.10.11-00:45:06 $-1 \quad 41 \quad 1 \quad 231 \quad-1=$ "schöne freud"

24 rlb 24.10.11-00:46:03 $-1 \quad 6 \quad 1 \quad 153$-1="schönheit freud"

25 rlb 24.10.11-00:46:17 $-1 \quad 4 \quad \begin{array}{llll}1 & 199 & -1=\text { "ästhetik freud" }\end{array}$

26 rlb 24.10.11-00:50:28 $-1 \quad 1 \quad 2 \quad 13 \quad 0=" 1280657 "$

27 rlb 24.10.11-00:51:03 $-1 \quad 1 \quad 2 \quad 11 \quad 0=" 587973 "$

28 rlb 24.10.11-01:03:14 $-1 \quad 3 \quad 1 \quad 342 \quad-1=$ "massenet leben"

29 plb 24.10.11-01:34:01 $-1 \quad 0 \quad 1 \quad 38$-1="Die Rechtsordnung der Bunderepublik Deutschland"

30 plb 24.10.11-01:34:54 $-1 \quad 0 \quad 1 \quad 28 \quad-1=$ "Avarenius" 


\section{B.1c: Umgewandelte Logfiles der UB Heidelberg vom 26.10.2011}

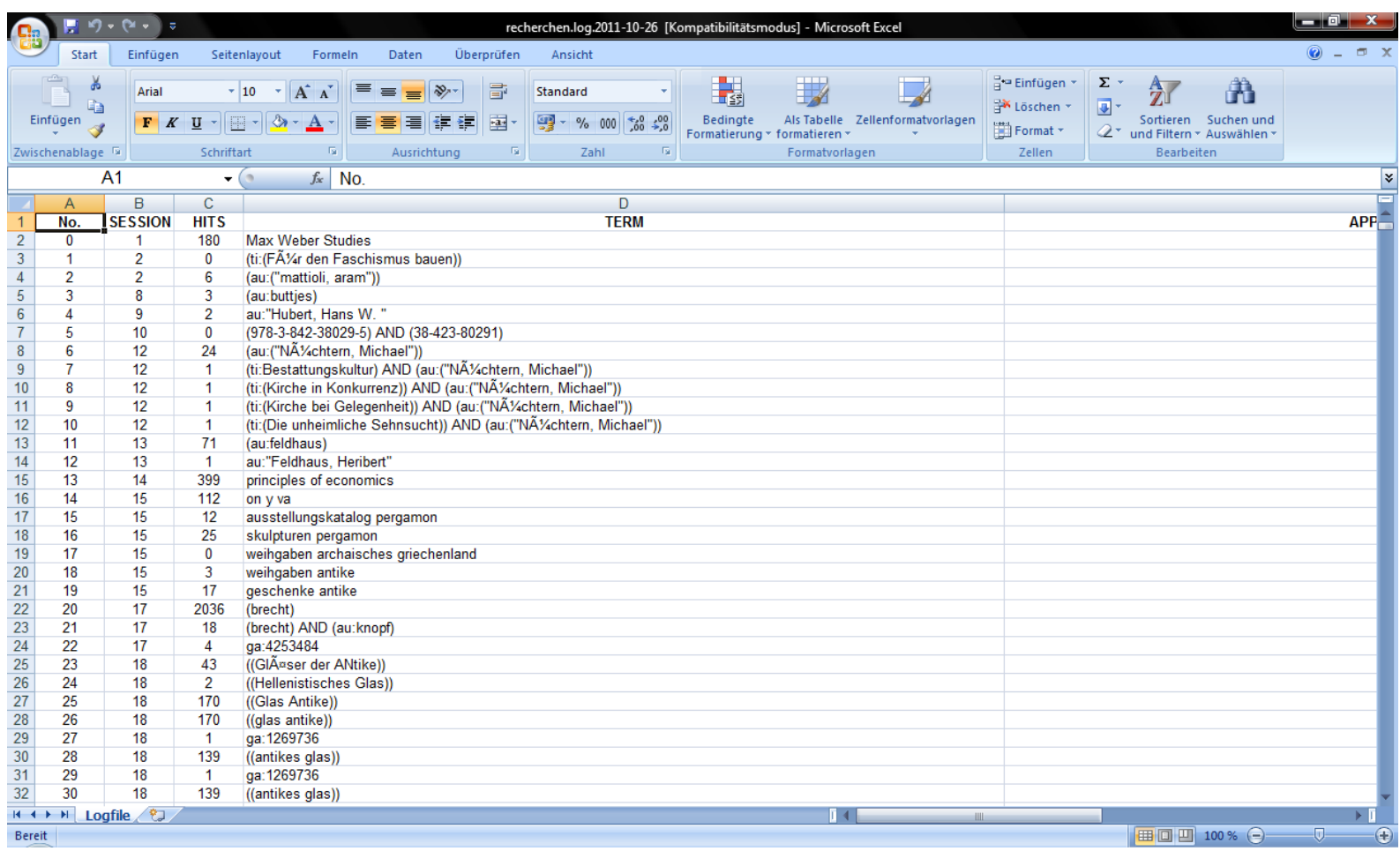

Abb. 18: Logfiles vom 26.10.2011 dargestellt mittels Excel

Ein vergrößerter Ausschnitt der aus Excel stammenden Logfiles:

No. SESSION HITS TERM

\begin{tabular}{|c|c|c|c|}
\hline 0 & 1 & 180 & Max Weber Studies \\
\hline 1 & 2 & 0 & (ti:(FÃ $1 / 4 r$ den Faschismus bauen)) \\
\hline 2 & 2 & 6 & (au:("mattioli, aram")) \\
\hline 3 & 8 & 3 & (au:buttjes) \\
\hline 4 & 9 & 2 & au:"Hubert, Hans W. " \\
\hline 5 & 10 & 0 & (978-3-842-38029-5) AND (38-423-80291) \\
\hline 6 & 12 & 24 & (au:("NÃ $1 / 4 c h t e r n$, Michael")) \\
\hline 7 & 12 & 1 & (ti:Bestattungskultur) AND (au:("NÃ¹/4chtern, Michael")) \\
\hline 8 & 12 & 1 & (ti:(Kirche in Konkurrenz)) AND (au:("NÃ¹/4chtern, Michael")) \\
\hline 9 & 12 & 1 & (ti:(Kirche bei Gelegenheit)) AND (au:("NÃ̃1/4chtern, Michael")) \\
\hline 10 & 12 & 1 & (ti:(Die unheimliche Sehnsucht)) AND (au:("NÃ 1/4chtern, Michael")) \\
\hline 11 & 13 & 71 & (au:feldhaus) \\
\hline 12 & 13 & 1 & au:"Feldhaus, Heribert" \\
\hline
\end{tabular}




$\begin{array}{llll}13 & 14 & 399 & \text { principles of economics } \\ 14 & 15 & 112 & \text { on y va } \\ 15 & 15 & 12 & \text { ausstellungskatalog pergamon } \\ 16 & 15 & 25 & \text { skulpturen pergamon } \\ 17 & 15 & 0 & \text { weihgaben archaisches griechenland } \\ 18 & 15 & 3 & \text { weihgaben antike } \\ 19 & 15 & 17 & \text { geschenke antike } \\ 20 & 17 & 2036 & \text { (brecht) } \\ 21 & 17 & 18 & \text { (brecht) AND (au:knopf) } \\ 22 & 17 & 4 & \text { ga:4253484 } \\ 23 & 18 & 43 & \text { ((GlÃaser der ANtike)) } \\ 24 & 18 & 2 & \text { ((Hellenistisches Glas)) } \\ 25 & 18 & 170 & \text { ((Glas Antike)) } \\ 26 & 18 & 170 & \text { ((glas antike)) } \\ 27 & 18 & 1 & \text { ga:1269736 } \\ 28 & 18 & 139 & \text { ((antikes glas)) } \\ 29 & 18 & 1 & \text { ga:1269736 } \\ 30 & 18 & 139 & \text { ((antikes glas)) }\end{array}$




\section{B.1d: Umgewandelte Logfiles der UB Heidelberg vom 05.11.2011}

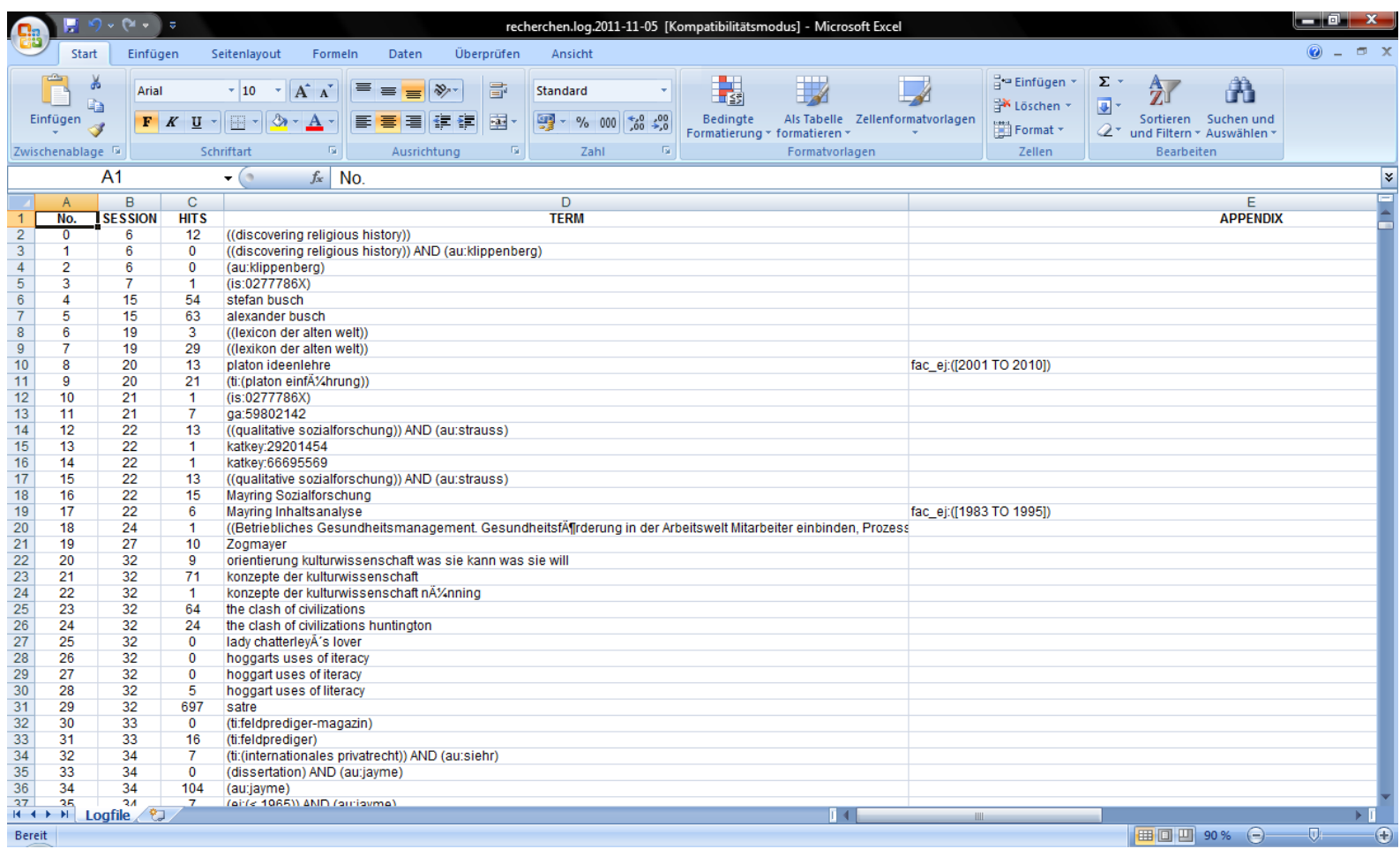

Abb. 19: Logfiles vom 05.11.2011 dargestellt mittels Excel

Ein vergrößerter Ausschnitt der aus Excel stammenden Logfiles:

No. SESSION HITS TERM

$\begin{array}{llll}0 & 6 & 12 & \text { ((discovering religious history)) } \\ 1 & 6 & 0 & \text { ((discovering religious history)) AND (au:klippenberg) } \\ 2 & 6 & 0 & \text { (au:klippenberg) } \\ 3 & 7 & 1 & \text { (is:0277786X) } \\ 4 & 15 & 54 & \text { stefan busch } \\ 5 & 15 & 63 & \text { alexander busch } \\ 6 & 19 & 3 & \text { ((lexicon der alten welt)) } \\ 7 & 19 & 29 & \text { ((lexikon der alten welt)) } \\ 8 & 20 & 13 & \text { platon ideenlehre } \\ 9 & 20 & 21 & \text { (ti:(platon einf } \tilde{A}^{1 / 4 h r u n g))} \\ 10 & 21 & 1 & \text { (is:0277786X) } \\ 11 & 21 & 7 & \text { ga:59802142 } \\ 12 & 22 & 13 & \text { ((qualitative sozialforschung)) AND (au:strauss) }\end{array}$




\begin{tabular}{|c|c|c|c|}
\hline 13 & 22 & 1 & katkey:29201454 \\
\hline 14 & 22 & 1 & katkey:66695569 \\
\hline 15 & 22 & 13 & ((qualitative sozialforschung)) AND (au:strauss) \\
\hline 16 & 22 & 15 & Mayring Sozialforschung \\
\hline 17 & 22 & 6 & $\begin{array}{l}\text { Mayring Inhaltsanalyse } \\
\text { ((Betriebliches Gesundheitsmanagement. GesundheitsfÃ } \text { Irderung in }\end{array}$ \\
\hline 18 & 24 & 1 & gestalten, Erfolge messen.)) \\
\hline 19 & 27 & 10 & Zogmayer \\
\hline 20 & 32 & 9 & orientierung kulturwissenschaft was sie kann was sie will \\
\hline 21 & 32 & 71 & konzepte der kulturwissenschaft \\
\hline 22 & 32 & 1 & konzepte der kulturwissenschaft nÃ $1 / 4 n n i n g$ \\
\hline 23 & 32 & 64 & the clash of civilizations \\
\hline 24 & 32 & 24 & the clash of civilizations huntington \\
\hline 25 & 32 & 0 & lady chatterleyÂ's lover \\
\hline 26 & 32 & 0 & hoggarts uses of iteracy \\
\hline 27 & 32 & 0 & hoggart uses of iteracy \\
\hline 28 & 32 & 5 & hoggart uses of literacy \\
\hline 29 & 32 & 697 & satre \\
\hline 30 & 33 & 0 & (ti:feldprediger-magazin) \\
\hline 31 & 33 & 16 & (ti:feldprediger) \\
\hline 32 & 34 & 7 & (ti:(internationales privatrecht)) AND (au:siehr) \\
\hline 33 & 34 & 0 & (dissertation) AND (au:jayme) \\
\hline 34 & 34 & 104 & (au:jayme) \\
\hline
\end{tabular}




\section{Anhang C: Fragebogen}

Der hier gezeigte Fragebogen ist ein Musterexemplar, so wie inn die Bibliotheken per Mail erhalten haben. Mit Absprache der Bibliotheken werden die Bögen anonym behandelt, so dass an dieser Stelle keine ausgefüllten Exemplare gezeigt werden können.

Die known-item search in der Bibliothekspraxis

Sie können die folgenden Fragen gerne subjektiv und stichwortartig beantworten. Ihre persönlichen Erfahrungen und Erkenntnisse sind besonders wichtig.

1) Known-item search vs. Thematische Suche

a) Suchen Ihre Nutzer prozentual gesehen überwiegend mit der known-item search (Suche nach etwas bekanntem: Titel, Verfasser, ISBN, Signatur) oder thematisch (z.B. mit Schlagwörtern)?

Bitte schätzen Sie

Known-item search: $\quad \%$

Thematische Suche \%

b) Recherchieren Sie persönlich im Auskunftsdienst häufiger mit einer known-item search oder einer Thematischen Suche?

Bitte schätzen Sie

Known-item search: $\quad \%$ 
Thematische Suche \%

2) Was wird Ihrer Meinung nach bei einer known-item search am häufigsten von den Benutzern abgefragt (z.B. Titelwörter, Verfasser, ISBN, Signatur)?

3) Wie häufig schätzen Sie, dass Benutzer mit einer Kombination aus mehreren Aspekten (z.B. Titel und Verfasser) suchen? Welche Kombination(en) halten Sie für die häufigste(n)?

4) Welcher Suchmodus (Einfache Suche / Erweiterte Suche) wird in Ihrer Institution am häufiger benutzt?

Bitte schätzen Sie

Einfache Suche $\quad \%$

Erweiterte Suche \%

5) Null-Treffer Ergebnisse treten Ihrer Meinung nach bei der known-item search in folgenden Fällen besonders häufig auf:

Bitte listen Sie kurz typische Gründe (z.B. Tippfehler, zu-viel-Eingeben) auf

6) Wie gehen die Suchenden bei einer 0-Trefferanzeige, nach Ihren Erfahrungen, vor (z.B. erneute Recherche, Verwendung von Kombinationen)? 
7) Gibt es Ihrer Meinung nach bekannte bzw. auffällige Vorgehensweisen von Nutzern bei einer known-item search?

Bitte listen Sie kurz typische Beispiele auf

8) Gibt es Punkte, die Sie an Ihrem Katalog gerne verbessern würden, um die known-item search für die Benutzer zu erleichtern?

Vielen Dank für das Ausfüllen dieses Fragebogens! 


\section{Quellenverzeichnis}

Dion Hoe-Lian Goh; Eng Pwey Lau (2006): In search of query patterns: A case study of a university OPAC, in: Information Processing \& Management 42, H. 5, S. 1316-1329

Dreßler, Juliane (2004): Suche in Bibliothekskatalogen: Eine Analyse der Suchanfragen an die Webkataloge der Universitätsbibliothek Freiburg. Diplomarbeit HdM http://opus.bsz-bw.de/hdms/volltexte/2005/538/pdf/Diplomarbeit V3.pdf (07.02.2012)

Frank, Silke (2006): Gestaltung von Benutzeroberflächen und Recherchemöglichkeiten bei OPACs: Sate of the art und Trends, in: Berliner Handreichungen zur Bibliotheks- und Informationswissenschaft, H. 188

Fuchs, Hans-Joachim (2010): Logfiles. Mail vom 28.11.2011

Greifeneder, Elke (2007): Online-Hilfen in OPACs: Analyse deutscher Universitätskataloge. Saarbrücken, VDM Verlag Dr. Müller

Guha, Tamal Kumar; Saraf, Veena (2005): OPAC usability: assessment through verbal protocol, in: The Electronic Library 23, Nr. 4, S. 463-473

IT Wissen (o.J.): IT Wissen: Das große Online-Lexikon für Informationstechnologie. Datacom Buchverlag http://www.itwissen.info/ (07.02.2012)

Kaske, Neal K. (1993): Research methodologies and transaction log analysis: Issues, questions, and a proposed model, in: Library Hi Tech 11 Nr. 2, S.79-86

Kneifel, Fabienne (2009): Mit Web 2.0 zum Online-Katalog der nächsten Generation. B.I.T.online - Innovativ 23. Wiesbaden, Dinges \& Frick 
Kurth, Martin (1993): Research methodologies and transaction log analysis: Issues, questions, and a proposed model, in: Library Hi Tech 11 Nr. 2, S. 98-104

Landesbibliothekszentrum Rheinland-Pfalz (2011)

http://www.lbz-rlp.de/cms/index.html

(07.02.2012)

Lang, Johannes und Kowatschew, Rossen (2002): Stemming. Präsentation präsentiert an der Universität Heidelberg, Institut für Computerlinguistik http://kontext.fraunhofer.de/haenelt/kurs/Referate/Kowatschew Lang/stemming.pdf (07.02.2012)

Liu, Weiling (2010): Understanding user OPAC searching habits through WebVoyagé log analysis: a comparative study of two cases. Presentation presented at University of Louisville

http://documents.el-una.org/459/1/ELUNA2010-Liu.pdf

(07.02.2012)

Malliari, Aphrodite; Kyriaki-Manessi, Daphne (2007): Users' behaviour patterns in academic libraries'OPACs: a multivariate statistical analysis, in: New Library World 108, Nr. 3/4, S. 107-122

Maylein, Leonhard (2010): Dauerbaustelle OPAC? HEIDI hält sich fit für die Zukunft http://www.vdb-online.org/veranstaltung/522/maylein heidi.pdf (07.02.2012)

Maylein, Leonhard (2012): Rechtschreibkorrektur in HEIDI. E-Mail vom 02.02.2012

Schneider, René (2009): OPACs, Benutzer und das Web, in: Zeitschrift für Bibliothekswesen und Bibliographie 56, H. 1, S. 9-19

Schulz, Ursula (1994): Was wir über OPAC-Nutzer wissen: Fehlertolerante Suchprozesse in OPACs, in: ABI-Technik 14, Nr. 4, S. 299-310

Serials Solutions (o.J.): The Summon ${ }^{\mathrm{TM}}$ Service 
http://www.serialssolutions.com/

(07.02.2012)

Staatsbibliothek zu Berlin (o.J.): StaBiKat: Online-Katalog der Staatsbibliothek zu

Berlin

http://stabikat.de//

(07.02.2012)

UB Bielefeld und Senst, Erik (2010): Tutorial zur Online-Recherche

http://www.ub.uni-bielefeld.de/help/tutorial/

(07.02.2012)

UB Heidelberg (o.J.): HEIDI: Katalog für die Bibliotheken der Universität Heidelberg http://www.ub.uni-heidelberg.de/helios/kataloge/heidi.html

(07.02.2012)

Ullrich, Hannah und Ruppert, Ato (2011): Katalog plus - die Freiburger Lösung zur Kombination von lokalem Katalog und globalem RDS-Index, in: Mitteilungsblatt des VDB-Regionalverbandes Südwest, Nr. 24, S. 10-16

Universitätsbibliothek der Humboldt-Universität zu Berlin (o.J.): Bibliotheksglossar

http://www.ub.hu-berlin.de/bibliotheksglossar

(07.02.2012)

Villén-Rueda, Luis; Senso, Jose A.; de Moya-Anegón, Félix (2007): The Use of OPAC in a Large Academic Library: A Transactional Log Analysis Study of Subject Searching, in: The journal of academic librarianship 33, Nr. 3, S. 327-337

Vogt, Renate (1999): „Es wurde keine Treffermenge gefunden“, in: ProLibris 4, H. 2, S. $90-94$

Weichert, Manfred (2002): „Gibt es auch Wahnsin“: Benutzungsuntersuchungen an einem Hamburger WebOPC, in: Bibliothek 26, Nr. 2, S. 142-149 
Weimar, Alexander (2004): Inhaltserschließung und OPAC-Retrieval am Beispiel des OPAC der Universitätsbibliothek Heidelberg. Diplomarbeit HdM http://opus.bsz-bw.de/hdms/volltexte/2005/495/pdf/Diplomarbeitweimar.pdf (07.02.2012)

Wiesenmüller, Heidrun (2012): Informationskompetenz und Bibliothekskataloge, in: Wilfried Sühl-Strohmenter (Hrsg.): Handbuch Informationskompetenz. De Gruyter (im Druck) 


\section{Eidesstattliche Versicherung}

Name: Krebs

Matrikel-Nr.: ${ }^{* \star \star * *}$
Vorname: Matthias

Studiengang: Bibliotheks- und Informationsmanagement (Bachelor)

Hiermit versichere ich, Matthias Krebs, an Eides statt, dass ich die vorliegende Bachelorarbeit mit dem Titel „Die known-item search in Bibliothekskatalogen - Nutzerverhalten, Probleme, Lösungsmöglichkeiten“ selbstständig und ohne fremde Hilfe verfasst und keine anderen als die angegebenen Hilfsmittel benutzt habe. Die Stellen der Arbeit, die dem Wortlaut oder dem Sinne nach anderen Werken entnommen wurden, sind in jedem Fall unter Angabe der Quelle kenntlich gemacht. Die Arbeit ist noch nicht veröffentlicht oder in anderer Form als Prüfungsleistung vorgelegt worden. Ich habe die Bedeutung der eidesstattlichen Versicherung und prüfungsrechtlichen Folgen (§ 26 Abs. 2 Bachelor-SPO der Hochschule der Medien Stuttgart) sowie strafrechtlichen Folgen (siehe unten) einer unrichtigen oder unvollständigen eidesstattlichen Versicherung zur Kenntnis genommen.

\section{Auszug aus dem Strafgesetzbuch (StGB)}

\section{§ 156 StGB Falsche Versicherung an Eides Statt}

Wer von einer zur Abnahme einer Versicherung an Eides Statt zuständigen Behörde eine solche Versicherung falsch abgibt oder unter Berufung auf eine solche Versicherung falsch aussagt, wird mit Freiheitsstrafe bis zu drei Jahren oder mit Geldstrafe bestraft.

Ort, Datum

Unterschrift 